\title{
Quaternary Ammonium Compounds (QACs) and Ionic Liquids (ILs) as Biocides: From Simple Antiseptics to Tunable Antimicrobials
}

\author{
Anatoly N. Vereshchagin*(D), Nikita A. Frolov (D), Ksenia S. Egorova, Marina M. Seitkalieva \\ and Valentine P. Ananikov *(D) \\ N. D. Zelinsky Institute of Organic Chemistry, Russian Academy of Sciences, Leninsky Prospect 47, \\ 119991 Moscow, Russia; nikitafrolov298@gmail.com (N.A.F.); egorova-ks@ioc.ac.ru (K.S.E.); \\ s_marina@ioc.ac.ru (M.M.S.) \\ * Correspondence: vereshchagin@ioc.ac.ru (A.N.V.); val@ioc.ac.ru (V.P.A.)
}

check for updates

Citation: Vereshchagin, A.N.; Frolov, N.A.; Egorova, K.S.; Seitkalieva, M.M.; Ananikov, V.P. Quaternary Ammonium Compounds (QACs) and Ionic Liquids (ILs) as Biocides: From Simple Antiseptics to Tunable Antimicrobials. Int. J. Mol. Sci. 2021, 22, 6793. https://doi.org/10.3390/ ijms22136793

Academic Editors: Iolanda Francolini and Antonella Piozzi

Received: 20 May 2021

Accepted: 16 June 2021

Published: 24 June 2021

Publisher's Note: MDPI stays neutral with regard to jurisdictional claims in published maps and institutional affiliations.

Copyright: (C) 2021 by the authors. Licensee MDPI, Basel, Switzerland. This article is an open access article distributed under the terms and conditions of the Creative Commons Attribution (CC BY) license (https:// creativecommons.org/licenses/by/ $4.0 /)$.

\begin{abstract}
Quaternary ammonium compounds (QACs) belong to a well-known class of cationic biocides with a broad spectrum of antimicrobial activity. They are used as essential components in surfactants, personal hygiene products, cosmetics, softeners, dyes, biological dyes, antiseptics, and disinfectants. Simple but varied in their structure, QACs are divided into several subclasses: Mono-, bis-, multi-, and poly-derivatives. Since the beginning of the 20th century, a significant amount of work has been dedicated to the advancement of this class of biocides. Thus, more than 700 articles on QACs were published only in 2020, according to the modern literature. The structural variability and diverse biological activity of ionic liquids (ILs) make them highly prospective for developing new types of biocides. QACs and ILs bear a common key element in the molecular structure-quaternary positively charged nitrogen atoms within a cyclic or acyclic structural framework. The state-of-the-art research level and paramount demand in modern society recall the rapid development of a new generation of tunable antimicrobials. This review focuses on the main QACs exhibiting antimicrobial and antifungal properties, commercial products based on QACs, and the latest discoveries in QACs and ILs connected with biocide development.
\end{abstract}

Keywords: quaternary ammonium compound; ionic liquid; antibacterial; antimicrobial; biocide

\section{Introduction}

For many years, quaternary ammonium compounds (QACs) have been included in most antiseptics and disinfectants and used in various areas, from household and agriculture to medicine and industry [1].

The COVID-19 pandemic that broke out in 2020 led to a significant increase in the widespread use of sanitizers, including QACs. Recent studies have shown that more than $90 \%$ of the dust samples analyzed during the pandemic contained QACs, and their average concentration doubled compared to the pre-COVID period [2]. It is to be expected that with the further progression of the pandemic, this number will increase, although the virucidal effect of QACs on SARS-CoV-2 requires further research [3].

The constant presence of subinhibitory concentrations of QACs on various working surfaces, together with the frequent use of QACs, increases the risk of the development of a resistant bacterial environment, which will lead to a plummet of the effectiveness of popular antiseptics and disinfectants. The solution to this problem can be found in the synthesis of new QACs, which exhibit superior antibacterial, antifungal, and antiviral properties.

The structure of QACs consists of a positively charged nitrogen atom with four or three substituents and one double bond. The core QAC structure can contain one (mono$\mathrm{QAC}$ ), two (bis-QAC), or more (multi-QAC, poly-QAC) charged nitrogen atoms, including 
those in heterocyclic compounds (piperidine, pyridine, imidazole, etc.). One or more of the substituents are usually long aliphatic chains containing at least ten carbon atoms. In the case of bis-QACs, multi-QACs, and poly-QACs, the structure that connects the charged nitrogen atoms (the head or nucleus fragment) is called a spacer or linker, and the alkyl chains extending from the heads (if they are present in the molecule) are called tails (Figure 1). QACs are generally water-soluble and stable. The counterion in these compounds usually does not affect the biological activity but often impacts the solubility of the biocide. The majority of the registered QACs contain chloride or bromide as anions. Due to their amphiphilic nature, QACs are able to form micelles. The critical concentration of micelle formation (CCM) is one of the important characteristics of these substances.

Mono-QAC

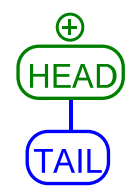

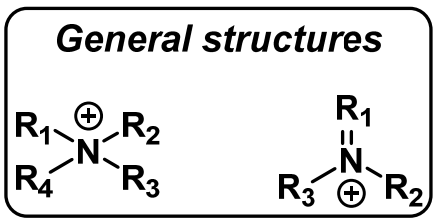
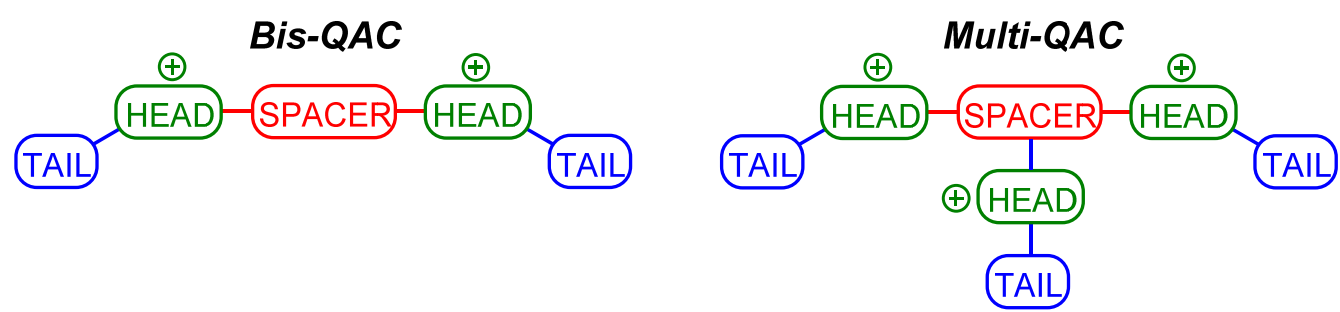

Figure 1. General structures and types of QACs.

The first studies of QACs as antibacterial agents were carried out at the beginning of the 20th century. Hexamethylenetetramine derivatives exhibited an in vitro bactericidal effect [4-6]. With the discovery of benzalkonium chloride (BAC) in 1935 [7], QACs found application in medical practice. Subsequently, the study of this class of compounds has led to the discovery of many valuable properties of QACs, due to which they are now used as surfactants, personal hygiene products, cosmetics, softeners, dyes, biological dyes, and, of course, antiseptics and disinfectants with a wide spectrum of action [8].

Therefore, QACs belong to the group of biocides-chemical compounds designed to neutralize, suppress, or prevent the action of harmful organisms by chemical or biological means [9]. As an example, in 2019, QACs accounted for ca. 11\% of the whole biocide market in the United States, which equals ca. \$192 million (Figure 2) [10].

The U.S. biocide market has grown by ca. $12 \%$ since 2016. The global trade of biocides, including QACs, is expected to grow by 3.9\% annually and to reach $\$ 10.5$ billion in 2027, thus evidencing the relevance and popularity of the topic. In other countries, similar trends can be expected due to the unquestionable significance of QACs.

Biocides are used in a wide variety of fields. Approximately $50 \%$ of biocide applications in the global market are in the water purification and paint industry (Figure 3) [10]. However, they also play an important role in the medical field [11]. 


\section{U. S. biocides market size, 2019}

( $\$ 1.8$ billion)

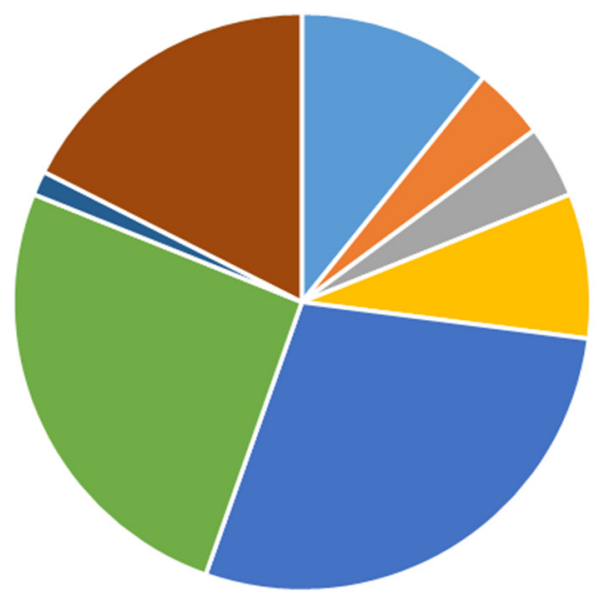

- Quaternary Ammonium Compounds

- Phenolic

- Nitrogen

- Glutaraldehyde

- Halogen Compounds

- Metallic Compounds

- Organic Acids

- Organosulfurs

Figure 2. Biocide market in USA.

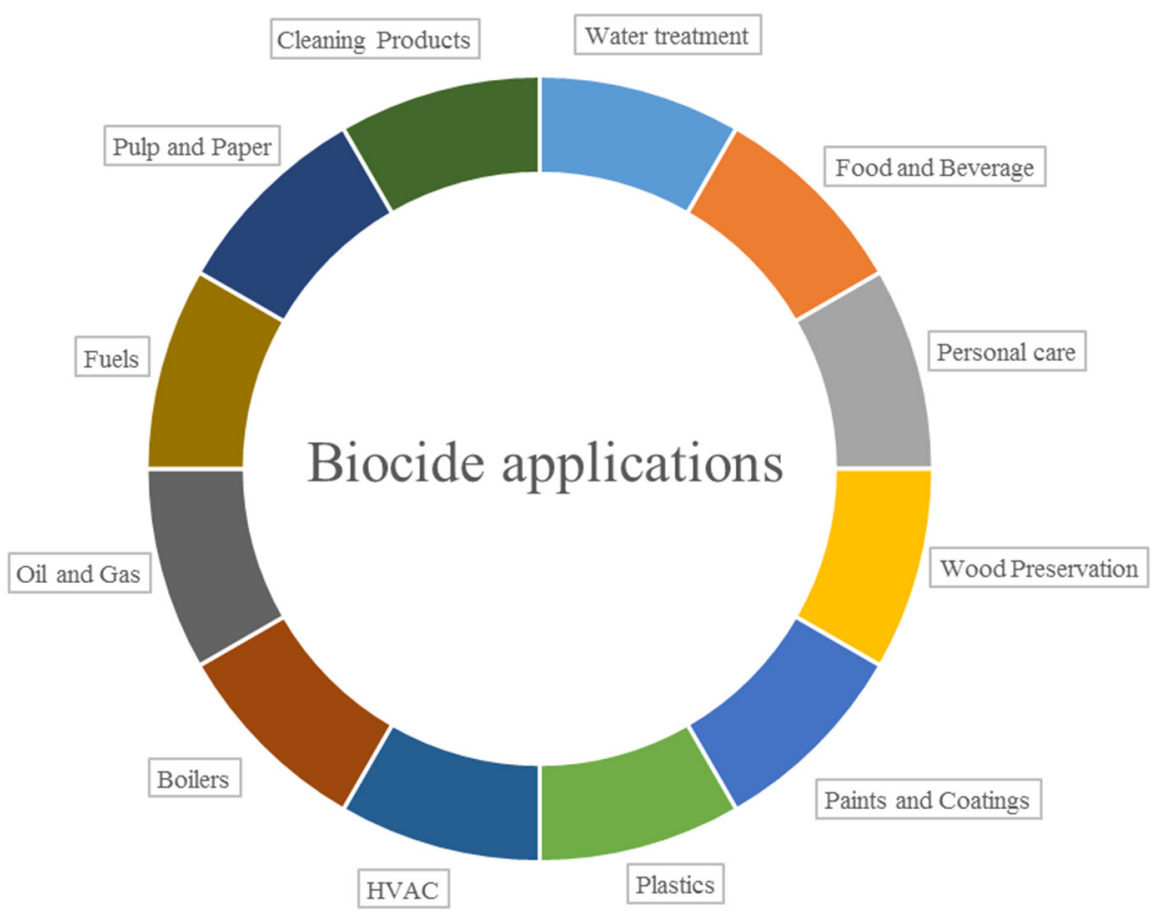

Figure 3. Biocide applications (HVAC—heating, ventilation, and air conditioning).

This review focuses on the main QACs exhibiting the characteristics of biocides, the latest discoveries and issues of this field, and is separated into two parts. The first part presents the main commercial QACs currently used as active substances in antiseptics and disinfectants. The second part describes the scientific research of this class of compounds. Due to the ever-increasing demand for new bactericides and fungicides, the search for compounds active against newly arisen resistant strains of pathogenic bacteria and fungi is one of the most important areas of modern pharmaceutics. Of special concern is the emergence of multidrug-resistant strains (so-called "superbugs"). Therefore, we also discuss the possibilities of applying ionic liquids (ILs) as antimicrobial compounds. ILs, some of which can be classified as QACs, comprise a class of substances with vast molecular diversity. These compounds have been shown to possess a wide range of biological activi- 
ties, including impressive antimicrobial properties [12,13]. A summary of the bactericidal and fungicidal activities of common ILs, bis-charged ILs, and poly-ILs is provided in the corresponding subsections.

\section{Antimicrobial Properties of QACs and ILs}

\subsection{Commercial QACS}

A significant step in the development of biologically active QACs was the discovery of benzalkonium chloride $\mathbf{1}$ (BAC) by Domagk in 1935. BAC is a mixture of mono-QACs with benzyl, methyl, and alkyl substituents with different chain lengths from $C_{8}$ to $C_{18}$ (Figure 4). This drug is the first active QAC compound approved by the US Environmental Protection Agency in 1947, and it has been widely used to date [14]. More details about the most important discoveries of that time in the QAC field can be found in the review by Rahn and Van Eseltine [15].

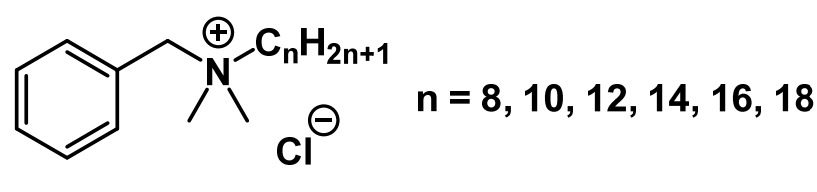

BAC (1)

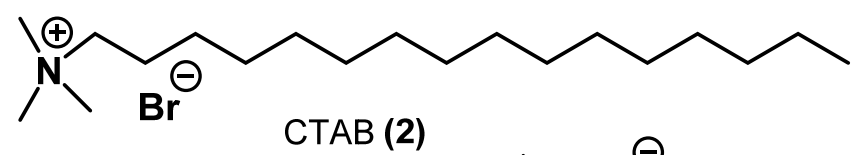<smiles>CCCCCCCCCC[N+](C)(C)CCCCCCCCCC</smiles><smiles>CCCCCCCCCCCCCC(=O)NCCC[N+](C)(C)Cc1ccccc1</smiles>

Figure 4. Commercial alkyl QACs.

The biological activity of benzalkonium salts depends on the length of the alkyl side chains. It is known that the $\mathrm{C}_{12}-\mathrm{C}_{14}$ compounds exhibit stronger bactericidal effects [16]. Due to its broad antibacterial activity and low toxicity, a mixture of benzalkonium derivatives is used in washing disinfectants for hands and face, mouthwashes, creams, and other cleansing and disinfecting products. BAC exhibits bactericidal activity against Staphylococcus, Streptococcus, Gram-negative bacteria (E. coli, Pseudomonas aeruginosa, Proteus, Klebsiella, etc.), anaerobic bacteria, fungi, and molds. It is also efficient against bacterial strains resistant to antibiotics and chemotherapeutic drugs; it inhibits Staphylococcus plasma coagulase and hyaluronidase. BAC prevents secondary wound infection with hospital strains [17]. In addition, a $0.2 \%$ aqueous solution of BAC was shown to inactivate the SARS-CoV-2 virus within $15 \mathrm{~s}$ [18].

Further study of this class of compounds led to the discovery of several currently widely known QACs with similar structures: alkyltrimethylammonium bromides. The most famous of them are cetyltrimethylammonium bromide (CTAB) 2 and dialkyldimethylammonium chloride, the main representative of the latter being dimethyldidecylammonium chloride (DDAC) 3 . The addition of the second long aliphatic chain increased the biological activity of the substance against $S$. aureus up to 8 times but, at the same time, increased its toxicity against red blood cells [8]. 
Miramistin 4 is a nonheterocyclic alkyl QAC and one of the most popular antibacterial agents in antiseptics used in Russia [19]. Miramistin demonstrates a moderate antiseptic effect against pathogenic fungi and viruses. Its aqueous solutions are used in the treatment of pyo-inflammatory diseases in surgery, obstetrics, gynecology, dermatology, urology, dentistry, and ophthalmology [20,21]. Miramistin-containing drugs have a pronounced bactericidal effect on Gram-positive (Staphylococcus spp., Streptococcus spp., Streptococcus pneumoniae, etc.), Gram-negative bacteria (Pseudomonas aeruginosa, Escherichia coli, Klebsiella spp., etc.), aerobic, and anaerobic bacteria, both in the form of monocultures and microbial associations, including hospital strains polyresistant to antibiotics. Moreover, miramistin demonstrates antiviral activities (hepatitis, HIV), prevents wound and burn contamination, and facilitates the recovery of damaged tissues [22].

Along with the majority of nonheterocyclic QACs on the antiseptic and disinfectant market, there are also examples of heterocyclic QACs, especially pyridine-based QACs (Figure 5).
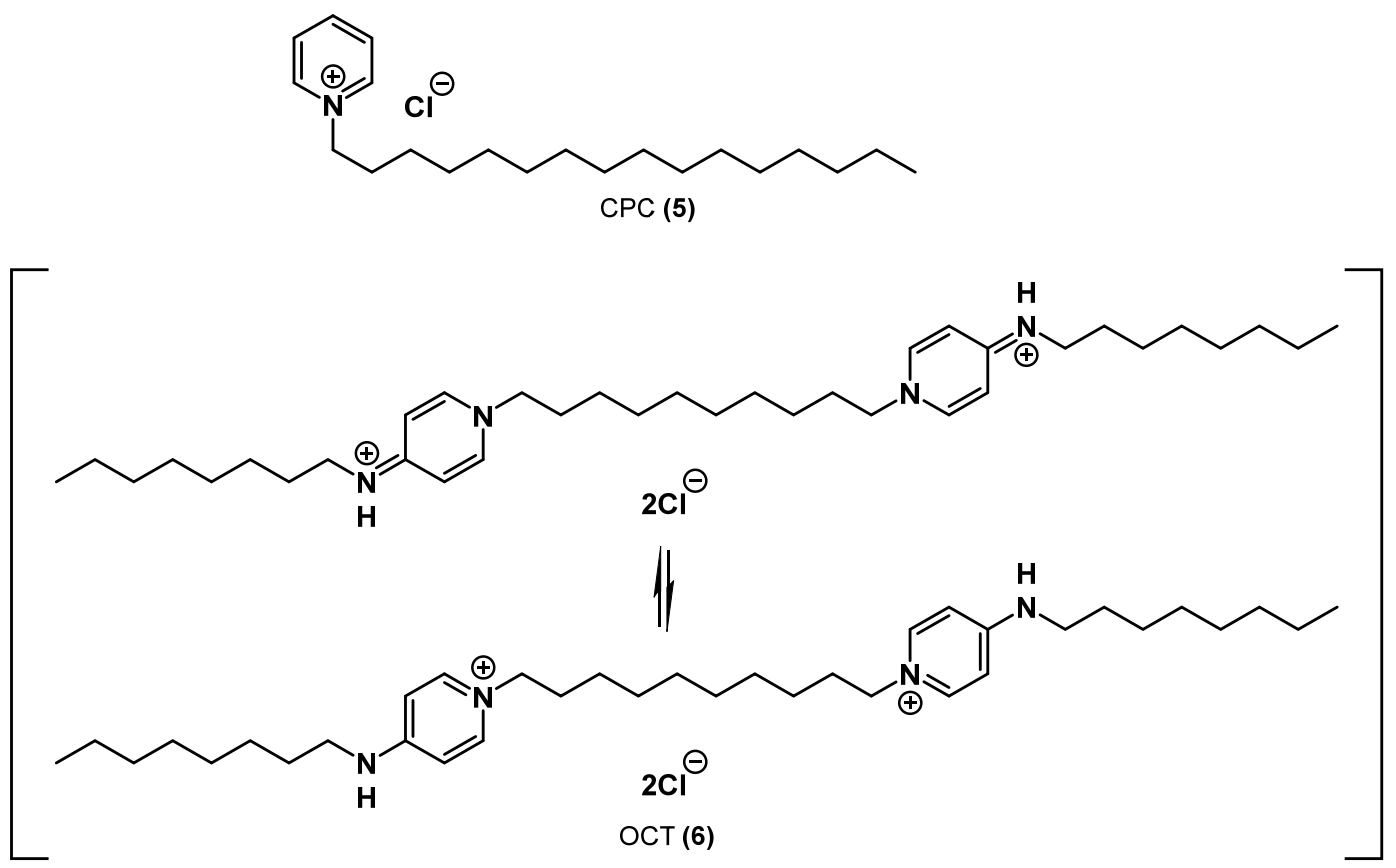

Figure 5. Commercial QACs based on pyridine.

The simplest of them is mono-QAC cetylpyridinium chloride $\mathbf{5}$ (CPC). First described shortly after BAC in 1939 [23], CPC has been extensively used in many mouthwashes and products for oral care [24]. In addition, CPC works as a preservative agent due to its outstanding inhibition properties of bacterial growth.

The second antiseptic of the subgroup is octenidine dihydrochloride 6 (OCT). Its dimeric structure is more complex than that of the other typical substances of this class. Here, two pyridinic nitrogen atoms linked via an alkyl bridge have alkylamine substituents in the para-position. OCT exists in pyridinic and imino forms. Due to its molecular structure, it demonstrates a broad spectrum of antibacterial activity, affecting S. aureus, S. epidermidis, P. mirabilis, K. pneumoniae, E. coli, P. aeruginosa, etc. [25]. Two cation-active centers divided by the long aliphatic carbon chain facilitate molecule binding to negatively charged surfaces of microbial cells. Strong interactions between octenidine and lipids (in particular, cardiolipins) in the bacterial cell membrane have been detected [26]. OCT has an intense residual effect on the skin, which is observed even $24 \mathrm{~h}$ after the last application. Due to its antimicrobial properties and skin compatibility, OCT can be used for various local applications where fast action and long-term effects are required, e.g., for disinfecting the skin of patients or treating acute and chronic wounds spontaneously colonized or locally 
infected by pathogenic bacteria. OCT can also be used for treating surgical equipment, injection sites of central catheters, infected root canals of teeth, candidiasis, acne, and nail infections [26-29].

A number of other biocides that play an important role in the modern market of antiseptics and disinfectants should also be mentioned. The antiseptics chlorhexidine bigluconate $\mathbf{7}$ (CHG), alexidine 9, and polyhexamethylene biguanide 8 (PHMB) (Figure 6) are guanidine derivatives from the cationic biocide family, as well as the abovementioned QACs [30].<smiles>N=C(NCCCCCCNC(=N)NC(=N)Nc1ccc(Cl)cc1)NC(=N)Nc1ccc(Cl)cc1</smiles><smiles>CCNC(=O)NC(=N[O-])NCCCCCC(C)(C)C</smiles>

Chlorhexidine bigluconate (7)<smiles>CCCCC(CC)CNC(=N)NC(=N)NCCCCCCNC(=N)NC(=N)NCC(CC)CCCC</smiles>

Figure 6. Commercial QACs-biguanide derivatives.

CHG is a symmetrical bis-biguanide connected by an alkyl chain; it carries two positive charges at physiological $\mathrm{pH}$. Developed in the early 1950s during the screening for antimalarial drugs, CHG has since recommended itself as a broad-spectrum antibacterial drug. CHG is one of the first antiseptics used on the skin and for decontamination of wounds. It is typically applied in the form of bigluconate, gluconate, dichloride, and acetate salts. Antiseptic drugs, which contain chlorhexidine bigluconate as an active substance, have a fairly wide spectrum of action. They are active against Gram-positive bacteria but not Gram-negative bacteria and mycobacteria or fungi. CHG is widely used in surgery and hand washing in the treatment of wound sepsis. It is also used in various oral hygiene products, as an anti-plaque agent, and in periodontal treatments. Similar activities were exhibited by aleksidine (Figure 6) [31-34].

PHMB is an alkyl biguanide polymer that can be used in a soluble form as chloride. It is an effective alternative to traditional antiseptics due to its low toxicity and superior antibacterial and antifungal activity [35]. It is used for treating swimming pools and fabrics, in cleaning products, and as a disinfectant for contact lenses and mouthwashes [36].

\subsection{The Latest Scientific Discoveries in the QAC Field}

The simplicity of synthesis, vast structural diversity, and high biological activity drive numerous scientific studies on QACs. Over the past 85 years, after the emergence of the class of cationic biocides, the number of publications on the topic has been arising significantly (Figure 7). According to SciFinder, more than 700 articles on QAC properties were published in 2020.

The scientific society proposes various synthetic procedures and applications for QACs, analyzes their structural fragments, and establishes the relations between the efficiency and molecular structure $[37,38]$. The last approach, known since the 19th century [39], is widely used in quantitative studies on various activities of chemical substances (QSAR, quantitative structure-activity relationship) [40]. 


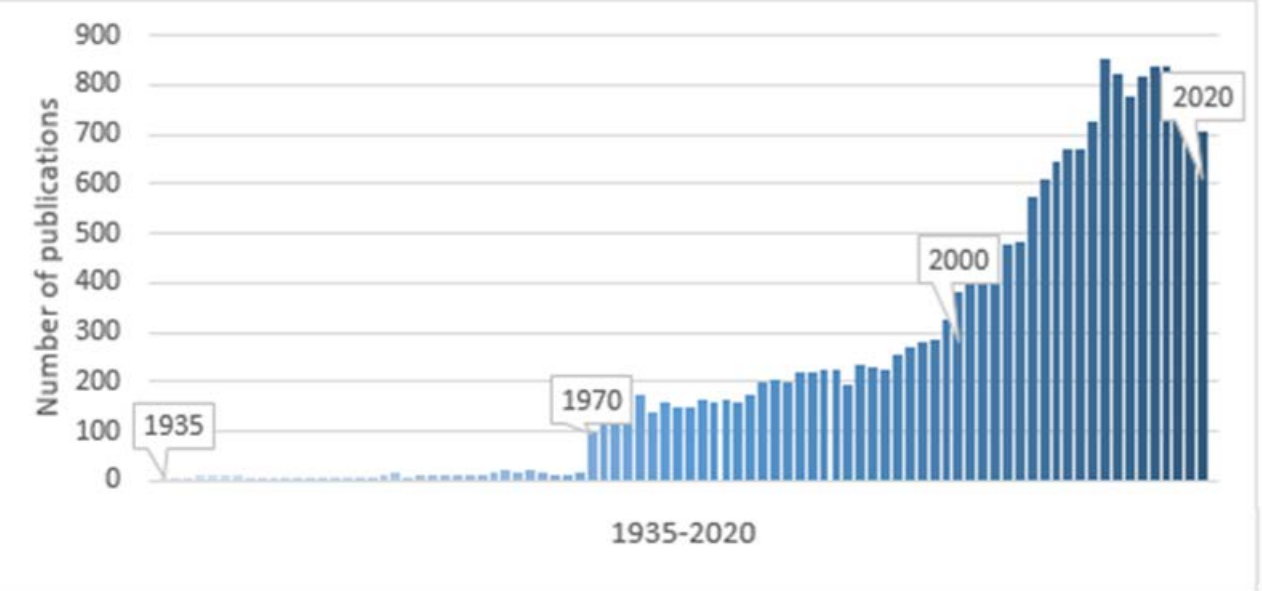

Figure 7. Number of publications involving QACs from 1935 to 2020 (SciFinder, January 2021).

Judging from the basic structure (Figure 1), one can change several parts in a given QAC to determine their impact on its activity:

Head. The number of charged nitrogen atoms (mono-, bis-, multi-QAC), as well as the head structure (non-heterocyclic, heterocyclic, aromatic), can be changed.

Spacer. The structure (aliphatic, aromatic, saturated, unsaturated, mixed, etc.) can be changed.

Tail. The structure (saturated, unsaturated, branched, unbranched) and the length of the aliphatic chain can be changed.

Substituents. A desired group can be introduced into any of the abovementioned fragments of the QAC molecule.

Hereafter, we will focus on representative examples of synthetic biocidal QACs obtained by various scientific groups in recent years. The effect of the structural fragments of the biocides on their biological activity will also be considered. The material is presented sequentially, depending on the QAC charge (mono-QAC, bis-QAC, poly-QAC). Additional information on studies on antimicrobial activity, surfactant properties, usage, and synthesis can be found in recent reviews on the topic [8,41-51].

\subsubsection{Single-Charged QACs (Mono-QACs)}

Thorsteinsson and colleagues developed "softer" analogues of the existing QAC biocides [52]. While "hard drugs" (CPC, BAC) are specified as drugs that are not subject to in vivo changes, "soft drugs" are metabolized to nontoxic compounds (Figure 8) [43].

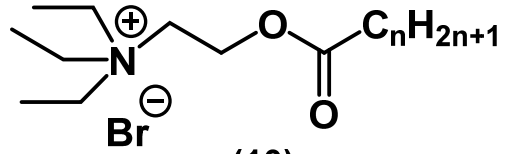

(10)<smiles></smiles>

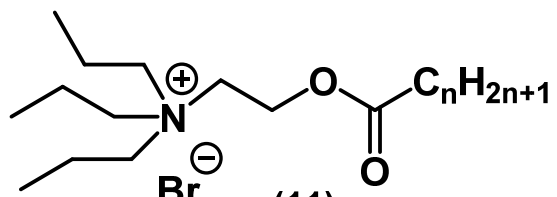

$\mathrm{Br}$ (11)

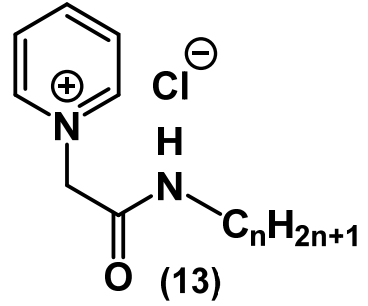

Figure 8. "Soft" mono-QACs.

Due to the introduction of amide and ether groups, the synthesized QAC molecules 10-13 are deactivated and decomposed into amides, fatty acids, and alcohols. Compounds 
without alkyl chains or with short chains $\left(C_{2}, C_{3}\right)$ were found to be inactive. Substances with $\mathrm{C}_{12}-\mathrm{C}_{18}$ alkyl tails exhibited antibacterial activity comparable to a known analog (BAC 1) against E. coli, S. aureus, and P. aeruginosa. Additionally, some compounds from series 11 showed activity against herpes simplex virus (HSV-1).

Miklas and colleagues carried out the synthesis and studied the biological properties of QACs based on camphorsulfonic acid (CSA) 14-16 (Figure 9) [53,54].

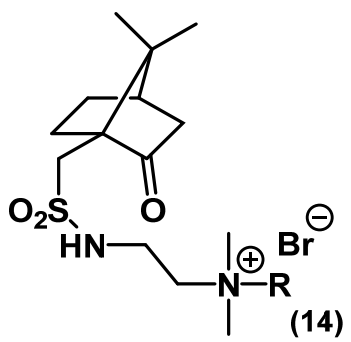

(14)

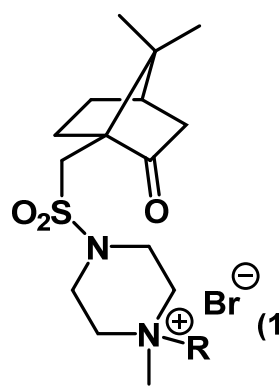

(15)

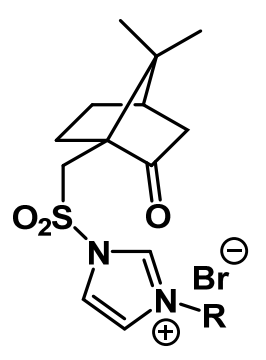

$\mathrm{R}=\mathrm{CH}_{2} \mathrm{COXC}_{n} \mathrm{H}_{2 n+1}, \mathrm{C}_{n} \mathrm{H}_{2 n+1}$

$\mathrm{X}=\mathrm{O}, \mathrm{N}$

(16)

Figure 9. CSA-based mono-QACs.

Upon changing the QAC core from ammonium to a less saturated heterocyclic structure (imidazole), the antimicrobial activity of the compounds gradually decreased. Salts with alkyl tails exhibited better activity than their ester and amide counterparts. The optimal chain length was found to be $\mathrm{C}_{12}-\mathrm{C}_{14}$.

In a recent work, Ali and colleagues developed new pyridine-based QACs from Schiff bases of nicotine hydrazines (Figure 10) [55].<smiles>[R]NNC(=O)c1ccc[n+](Br)c1</smiles><smiles>C/C=C/c1ccccc1OC</smiles><smiles>C/C=C/c1ccccc1</smiles><smiles>COc1ccc(C)cc1O</smiles><smiles>Cc1cccc(O)c1</smiles>

Figure 10. Mono-QACs containing hydrazide bridges.

These substances had good water solubility, most likely due to the presence of hydrazide groups. Despite the shorter alkyl chains (compared to typical QACs), a series of substances 17 showed high activity against colonies and biofilms of E. coli and S. aureus. According to this study, the presence of donor groups in the phenyl ring of the $\mathrm{R}$ substituent increased the bactericidal activity.

In the works of Liu and colleagues, the effect of combining two biocidal fragments (N-chloramines and alkyl QACs) in one molecule 18-19 on bactericidal properties was studied (Figure 11) [56-58].

Chloramines act on bacterial cells through the oxidative transfer of chlorine to biological receptors which leads to cell lysis. The attachment of the QAC molecule with a positive charge allowed anchoring of the $\mathrm{N}$-chloramine moiety on the surface of the bacterial cell, thus enhancing the effect [56]. The introduction of a long alkyl chain into the compound leads to the rupture of the bacterial membrane, penetration of the biocide into the cell, 
and a subsequent enhancement of the bactericidal effect [57,58]. At the same time, Li and colleagues combined a pyridinic QAC with $N$-chloramine 20 (Figure 11). The antibacterial activity of this compound was similar to that presented by Liu [59].

In the works of Wang and Hou, a similar approach to changing the structure of QAC by adding biologically active fragments to the molecule was used (Figure 12) $[60,61]$.<smiles>CC[N+](C)(C)CCn1cc(COC2CC(C)(C)N(Cl)C(C)(C)C2)nn1</smiles>

(18)<smiles>CCCCCCCN1C(=O)N(Cl)C(C)(C)C1=O</smiles>

(19)<smiles>CC(C)([O-])N1C(=O)N(Cl)C(C)(C)C1=O</smiles>

$\mathrm{n}=3,6,8,12$

Figure 11. Mono-QACs containing N-chloramines.<smiles></smiles>

(21)<smiles>CCN(CCO)CCO</smiles>

$m=12,14,16,18$<smiles>[R]c1ccc(-c2nnc(SCCCCC(C)(C)C(C)(C)C(F)(F)F)[nH]2)cc1</smiles>

(23)<smiles>Cc1nnc(SCCCCC(C)(C)C[Hg](C)(Br)CCO)o1</smiles>

$\mathrm{n}=4,6,8,10$

Figure 12. Mono-QACs containing hydroxyl groups.

Initially, guided by the hypothesis that hydroxy groups should stimulate membrane penetration and cell destruction, a series of hydroxy-QACs 22 with different alkyl chain lengths was synthesized. All the resulting compounds exhibited lower antibacterial activity than CHG; they also demonstrated antifungal activity with an optimal tail length of $C_{12}$. It should be noted that the toxicity of the compounds correlated with their activity [60]. Then, a fragment of oxadiazole derivatives $\mathbf{2 3 - 2 4}$, benzothiazole $(X=S) \mathbf{2 1}$, and benzoxazole $(X=O) 21$ was introduced into the QAC molecule, which led to an increase in bactericidal and fungicidal activity and a decrease in toxicity in epithelial cells and erythrocytes [61].

Bogdanov and colleagues explored the microbiological effect of isatin-based QACs (Figure 13) [62].

As seen from the figure, the structures of these ammonium 25 and pyridine $26-27$ salts contain no long alkyl chains. Therefore, the cytotoxicity of these compounds is significantly lower than that of typical QACs. However, the antibacterial activity is markedly reduced in the absence of quaternary nitrogen tails. Thus, none of the compounds from this series showed a biocidal effect against the Gram-negative bacteria E. coli and P. aeruginosa. 
On the other hand, these salts inhibited the growth of Gram-positive bacteria (S. aureus and B. cereus) and fungi (C. albicans) at concentrations comparable to modern antibiotics (chloramphenicol and norfloxacin). Overall, QACs with pyridinium nuclei and donor substituents in the aromatic part of isatin 27 turned out to be more active than the others.

Rusew and colleagues presented a work, in which long lipophilic tails in QACs were replaced by more compact aryl-containing substituents (Figure 14) [63].<smiles>[R]c1cc2c(c([R])c1[R])NC(=O)/C2=N/NC(=O)C[N+](C)(C)C</smiles>

(25)

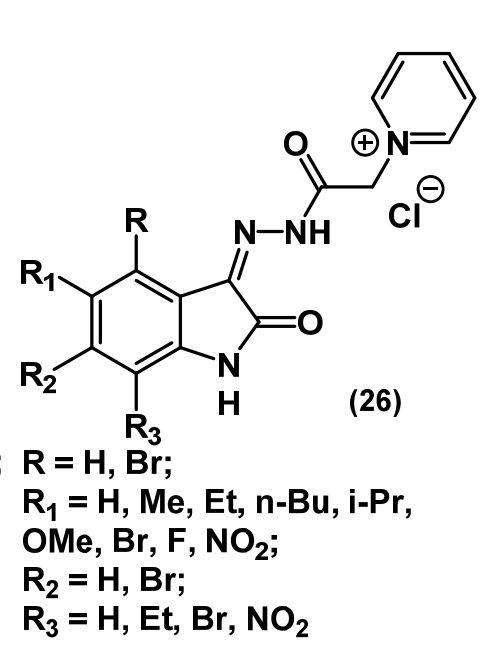

Figure 13. Isatin-based mono-QACs.

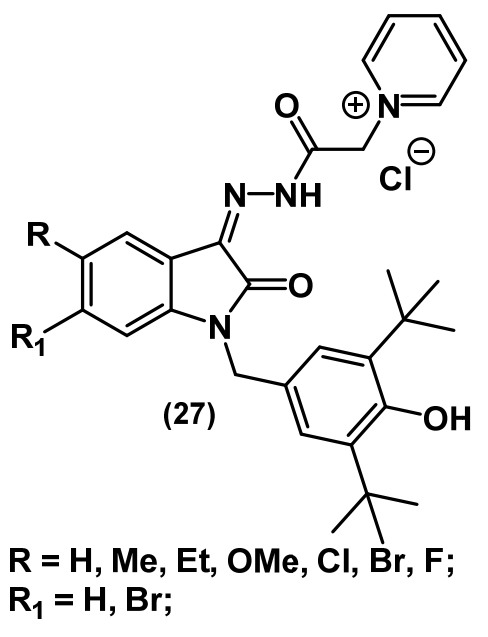

$\mathrm{R}=\mathrm{H}, \mathrm{Me}$,
$\mathrm{R}_{\mathbf{1}}=\mathrm{H}, \mathrm{Br} ;$

$\mathrm{R}=\mathrm{H}, \mathrm{Me}, \mathrm{Et}, \mathrm{n}-\mathrm{Bu}$, i-Pr, OMe, $\mathrm{Cl}, \mathrm{Br} ; \mathrm{R}=\mathrm{H}, \mathrm{Br}$

$\mathrm{R}_{\mathbf{1}}=\mathrm{H}, \mathrm{Br} ; \mathrm{R}_{\mathbf{2}}=\mathrm{H}, \mathrm{Me}, \mathrm{Et}, \mathrm{Br}, \mathrm{NO}_{\mathbf{2}}$<smiles>CCCC[n+]1ccc(N2CCCC2)cc1</smiles>

(28)<smiles>[R]C(=O)C[n+]1ccc(N2CCCC2)cc1</smiles>

(29)<smiles>Cc1ccc(-c2ccccc2)cc1</smiles><smiles>COc1ccc(C)c(OC)c1</smiles><smiles>Cc1ccc(C)cc1</smiles>

Figure 14. Mono-QACs containing aryl substituents.

The results of a broad antibacterial screening appeared to be nontypical for cationic biocides. Compounds with biphenyl and 1,3-dimethoxyphenyl 29 substituents selectively inhibited the growth of E. coli (Gram-negative) and S. aureus (Gram-positive) but no other Gram-positive and Gram-negative bacteria. In a quantitative sense, the inhibiting zones of these substances were similar to kanamycin.

Kuca and Soukup studied the biological activity of picolinic QAC with methyl substituents 30 (Figure 15) [64]. 

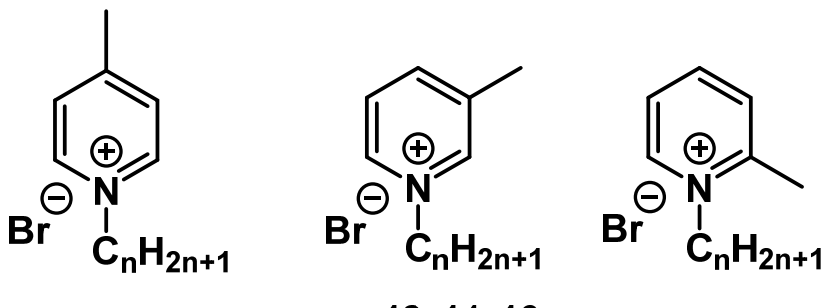

$\mathrm{n}=12,14,16$

(30)

Figure 15. Picolinic mono-QACs.

It was found that the position of the substituent did not significantly affect the biocidal effect of methylpicolinates, possibly due to the small size of the methyl substituent. Overall, picolinates showed a comparable or even superior bacteriostatic effect compared to BAC on a wide range of pathogens. The optimal tail length was $C_{14}-C_{16}$, and higher activity was observed in Gram-positive bacteria than in Gram-negative bacteria, as with most QACs.

Shtyrlin and his colleagues created a pyridoxine-based QAC library, including bisderivatives, which will be discussed in the corresponding part of the review (Figure 16) [65-70].<smiles>CC1(C)OCc2nc(CCl)c3c(c2CO1)COC(C)(C)O3</smiles>

(31)<smiles>CC1(C)OCc2c(CO)c(CO)nc(CCl)c2O1</smiles>

(32)<smiles>[R2]C1([R2])OCc2c(C[N+](C)(C)C)cnc(C)c2O1</smiles>

$\mathrm{R}_{1}=\mathrm{H}, \mathrm{CH}_{3}, \mathrm{C}_{2} \mathrm{H}_{5}$, $\mathrm{C}_{3} \mathrm{H}_{9}, \mathrm{C}_{4} \mathrm{H}_{10}, \mathrm{C}\left(\mathrm{CH}_{3}\right)_{3}$, $\mathrm{C}_{8} \mathrm{H}_{17}, \mathrm{CH}\left(\mathrm{CH}_{3}\right) \mathrm{C}_{9} \mathrm{H}_{19}$; $\mathbf{R}_{\mathbf{2}}=\mathrm{H}, \mathrm{CH}_{\mathbf{3}}$.

(33)<smiles>[R2]C1([R2])Cc2c(C)nc3c(c2CO1)OC([3H])([13CH2][3H])[NH+]([3H])C3</smiles>

$$
\begin{aligned}
& \mathrm{R}_{1}=\mathrm{CH}_{3}, \mathrm{C}_{2} \mathrm{H}_{5}, \\
& \mathrm{C}_{3} \mathrm{H}_{7}, \mathrm{C}_{5} \mathrm{H}_{11} ; \\
& \mathrm{R}_{2}=\mathrm{H}, \mathrm{CH}_{3} .
\end{aligned}
$$<smiles>[R3]C(=O)NC(C)[N+](C)(C)Cc1cnc(C)c2c1COC([R2])([R2])O2</smiles>

$\mathrm{n}=2,3$;

$\mathbf{R}_{1}=\mathrm{CH}_{3}, \mathrm{C}_{3} \mathrm{H}_{7}, \mathrm{C}_{5} \mathrm{H}_{11}$,

$\mathrm{C}_{8} \mathrm{H}_{17}, \mathrm{CH}\left(\mathrm{CH}_{3}\right) \mathrm{C}_{8} \mathrm{H}_{17}$;

$\mathbf{R}_{\mathbf{2}}=\mathbf{H}, \mathbf{C H}_{3}$;

$\mathrm{R}_{3}=\mathrm{C}_{5} \mathrm{H}_{11}, \mathrm{C}_{11} \mathrm{H}_{23}$,

$\mathrm{C}_{13} \mathrm{H}_{27}, \mathrm{C}_{15} \mathrm{H}_{31}, \mathrm{C}_{17} \mathrm{H}_{35}$.

(35)

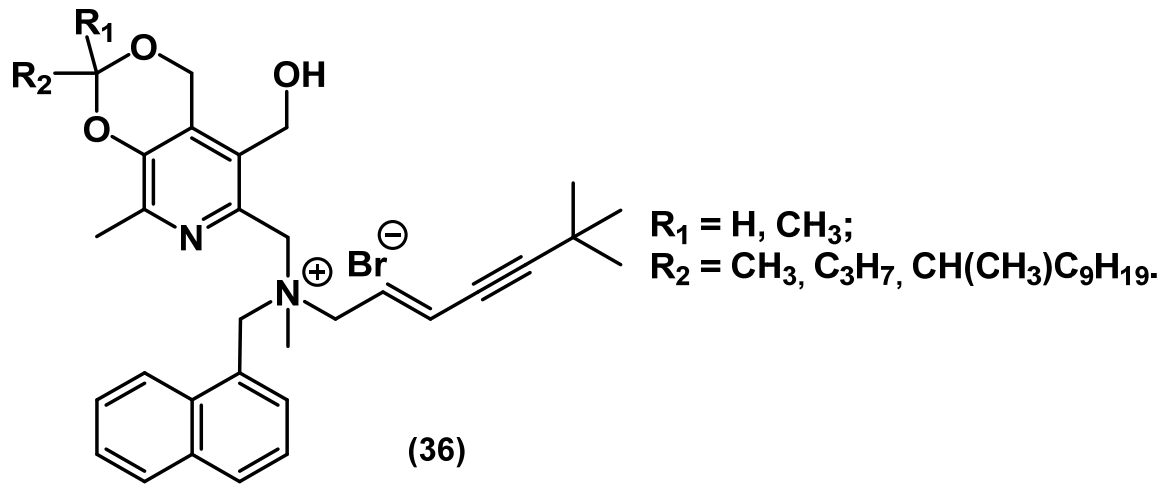

Figure 16. Pyridoxin-based mono-QACs. 
Pyridoxin functional derivatives 31-36 exhibited a broad spectrum of antibacterial and antifungal activity; at that time, they were more active against Gram-positive bacteria than Gram-negative bacteria. It should be mentioned that a combination of the antifungal drug terbinafine with pyridoxin-based QAC 36 was efficient against mixed colonies of pathogenic bacteria and fungi. This example proved the advantage of combining two different biocide fragments in one molecule.

A significant contribution to the development of QACs as a class of cationic biocides was made by the groups of Wuest and Minbiole (Figure 17) [71-76].

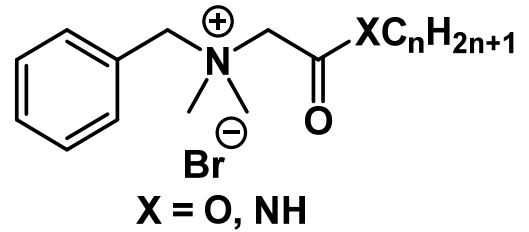

(37)<smiles>CN1CCN(C)C[N+](C)(Br)CC1</smiles>

(40)<smiles>C=CC1C2CCN(C(Br)Br)[C@@H]1C2C(=O)O</smiles>

(38)

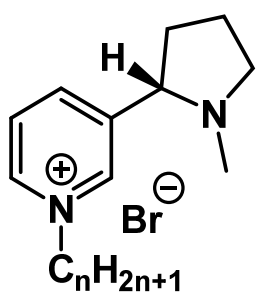

(39)<smiles>CN(C)CC[N+](C)(C)[O-]</smiles>

(41)

Figure 17. Mono-QACs from Wuest's and Minbiole's works.

It was found that close structural analogs of BAC 37 containing amide and ester groups exhibited comparable activity and lower toxicity than BAC [76]. QAC derivatives of natural compounds (quinine 38 and nicotine 39) demonstrated a wide spectrum of antibacterial action, thus justifying the search for other platforms of natural origin to expand the library of active QAC compounds [74].

An overview of the antibacterial activity of mono-QACs, analyzed in the review, is shown in Table 1.

2.2.2. Common Ionic Liquids and Ionic Liquids with Active Pharmaceutical Ingredients (API-ILs)

ILs are organic salts that generally exist in liquid form at a wide range of temperatures. The most common ILs are composed of a bulky organic cation and a more compact anion (Figure 18). Due to its broad applications in chemistry, this class of compounds has been studied thoroughly, and the chemical and physicochemical properties, as well as biodegradation potential, of various ILs have been determined [12,77]. 
Table 1. Antimicrobial activity of mono-QACs *

\begin{tabular}{|c|c|c|c|c|c|c|}
\hline $\begin{array}{c}\text { Series/ } \\
\text { Compound }\end{array}$ & Strain & $\mathrm{MIC}, \mathrm{mg} \cdot \mathrm{L}^{-1}$ & $\mathrm{MBC}, \mathrm{mg} \cdot \mathrm{L}^{-1}$ & Method & Notes & Ref. \\
\hline \multirow{4}{*}{10} & E. faecalis ATCC 29212 & 8 & 16 & \multirow{4}{*}{ Microtiter dilution } & & \multirow{4}{*}{52} \\
\hline & S. aureus ATCC 25923 & 2 & 4 & & & \\
\hline & E. coli ATCC 25922 & 64 & 64 & & & \\
\hline & P. aeruginosa ATCC 27853 & 250 & 250 & & & \\
\hline \multirow{3}{*}{11} & E. faecalis ATCC 29212 & 4 & 8 & \multirow{3}{*}{ Microtiter dilution } & \multirow{3}{*}{$\begin{array}{c}\text { Active towards herpes simplex } \\
\text { virus }\end{array}$} & \multirow{3}{*}[52]{} \\
\hline & E. coli ATCC 25922 & 125 & 250 & & & \\
\hline & P. aeruginosa ATCC 27853 & 250 & 1000 & & & \\
\hline \multirow{4}{*}{12} & E. faecalis ATCC 29212 & 1 & 4 & \multirow{4}{*}{ Microtiter dilution } & & \multirow{4}{*}[52]{} \\
\hline & S. aureus ATCC 25923 & $<0.25$ & 1 & & & \\
\hline & E. coli ATCC 25922 & 250 & 250 & & & \\
\hline & P. aeruginosa ATCC 27853 & 500 & 500 & & & \\
\hline \multirow{3}{*}{13} & S. aureus ATCC 25923 & $<0.25$ & 4 & \multirow{3}{*}{ Microtiter dilution } & & \multirow{3}{*}[52]{} \\
\hline & E. coli ATCC 25922 & 1000 & $>2000$ & & & \\
\hline & P. aeruginosa ATCC 27853 & 1000 & $>2000$ & & & \\
\hline \multirow{3}{*}{14} & S. aureus ATCC 6538 & $1.05 \mu \mathrm{M}$ & & \multirow{3}{*}{ Broth microdilution } & & \multirow{3}{*}[54]{} \\
\hline & E. coli CNCTC $377 / 79$ & $2.2 \mu \mathrm{M}$ & & & & \\
\hline & C. albicans CCM 8186 & $1.05 \mu \mathrm{M}$ & & & & \\
\hline \multirow{3}{*}{15} & S. aureus ATCC 6538 & $5.2 \mu \mathrm{M}$ & & \multirow{3}{*}{ Broth microdilution } & & \multirow{3}{*}[54]{} \\
\hline & E. coli CNCTC $377 / 79$ & $41.2 \mu \mathrm{M}$ & & & & \\
\hline & C. albicans CCM 8186 & $164.9 \mu \mathrm{M}$ & & & & \\
\hline
\end{tabular}


Table 1. Cont.

\begin{tabular}{|c|c|c|c|c|c|c|}
\hline $\begin{array}{l}\text { Series/ } \\
\text { Compound }\end{array}$ & Strain & $\mathrm{MIC}, \mathrm{mg} \cdot \mathrm{L}^{-1}$ & $\mathrm{MBC}, \mathrm{mg} \cdot \mathrm{L}^{-1}$ & Method & Notes & Ref. \\
\hline \multirow{3}{*}{16} & S. aureus ATCC 6538 & $5.4 \mu \mathrm{M}$ & & \multirow{3}{*}{ Broth microdilution } & & \multirow{3}{*}[53]{} \\
\hline & E. coli CNCTC $377 / 79$ & $144.1 \mu \mathrm{M}$ & & & & \\
\hline & C. albicans CCM 8186 & $5.4 \mu \mathrm{M}$ & & & & \\
\hline \multirow{2}{*}{17} & S. aureus ATCC 6538 & $75 \%$ (percent of inhibition, $250 \mathrm{mg} \cdot \mathrm{L}^{-1}$ ) & & \multirow{2}{*}{ Broth microdilution } & \multirow{2}{*}{$\begin{array}{l}\text { Active towards bacterial } \\
\text { biofilms }\end{array}$} & \multirow{2}{*}[55]{} \\
\hline & E. coli CNCTC $377 / 79$ & $80 \%$ (percent of inhibition, $250 \mathrm{mg} \cdot \mathrm{L}^{-1}$ ) & & & & \\
\hline \multirow{3}{*}{18} & E. coli ATCC 25922 & & $3 \min (\mathrm{Tk}) / 141 \mu \mathrm{M}$ & & & \multirow{3}{*}[58]{} \\
\hline & $\begin{array}{c}\text { multidrug-resistant (MDR) } \\
\text { P. aeruginosa } 73104\end{array}$ & & $<1 \min (\mathrm{Tk}) / 141 \mu \mathrm{M}$ & & & \\
\hline & $\begin{array}{l}\text { wild-type P. aeruginosan } \\
\text { PA01 }\end{array}$ & & $3 \min (\mathrm{Tk}) / 141 \mu \mathrm{M}$ & & & \\
\hline \multirow{3}{*}{19} & $\begin{array}{l}\text { methicillin-resistant } S \text {. } \\
\text { aureus (MRSA) } 70065\end{array}$ & & $\begin{array}{l}3 \min (\mathrm{Tk} \text { (time to } \\
\mathrm{kill})) / 141 \mu \mathrm{M}\end{array}$ & & & \multirow{3}{*}[58]{} \\
\hline & $\begin{array}{l}\text { multidrug-resistant (MDR) } \\
\text { P. aeruginosa } 73104\end{array}$ & & $5 \mathrm{~min}(\mathrm{Tk}) / 141 \mu \mathrm{M}$ & & & \\
\hline & $\begin{array}{c}\text { wild-type P. aeruginosan } \\
\text { PA01 }\end{array}$ & & $5 \min (\mathrm{Tk}) / 141 \mu \mathrm{M}$ & & & \\
\hline \multirow{2}{*}{20} & S. aureus & $\begin{array}{l}99 \% \text { (reduction, contact time }-5 \mathrm{~min} \text {, } \\
20 \mathrm{ppm})\end{array}$ & & \multirow{2}{*}{ AATCC test } & & \multirow{2}{*}[59]{} \\
\hline & E. coli & $\begin{array}{l}100 \% \text { (reduction, contact time }-5 \mathrm{~min}, \\
20 \mathrm{ppm} \text { ) }\end{array}$ & & & & \\
\hline
\end{tabular}


Table 1. Cont.

\begin{tabular}{|c|c|c|c|c|c|c|}
\hline $\begin{array}{c}\text { Series/ } \\
\text { Compound }\end{array}$ & Strain & $\mathrm{MIC}, \mathrm{mg} \cdot \mathrm{L}^{-1}$ & $\mathrm{MBC}, \mathrm{mg} \cdot \mathrm{L}^{-1}$ & Method & Notes & Ref. \\
\hline \multirow{10}{*}{21} & S. aureus & 6.25 & 6.25 & \multirow{10}{*}{ Broth tube dilution } & & \multirow{10}{*}{ [61] } \\
\hline & $a-H$-tococcus & 12.5 & 12.5 & & & \\
\hline & $b$-H-tococcus & 1.56 & 3.125 & & & \\
\hline & E. coli & 25 & 25 & & & \\
\hline & P. aeruginosa & 25 & 25 & & & \\
\hline & P. vulgaris & 25 & 25 & & & \\
\hline & C. albicans & 6.25 & 6.25 & & & \\
\hline & C. mandshurica & 1.56 & 6.25 & & & \\
\hline & P. piricola & 3.125 & 3.125 & & & \\
\hline & A. niger & 3.125 & 6.25 & & & \\
\hline \multirow[b]{2}{*}{22} & S. aureus & $22.4 \mathrm{~mm}(\mathrm{IZ}, 500$ ppm) & & \multirow[b]{2}{*}{ Disk diffusion } & & \multirow[b]{2}{*}[60]{} \\
\hline & B. subtilis & $17 \mathrm{~mm}(\mathrm{IZ}, 500 \mathrm{ppm})$ & & & & \\
\hline \multirow{10}{*}{23} & S. aureus & 6.25 & 6.25 & \multirow{10}{*}{ Broth tube dilution } & & \multirow{10}{*}[61]{} \\
\hline & $a$-H-tococcus & 6.25 & 6.25 & & & \\
\hline & $b$-H-tococcus & 1.56 & 1.56 & & & \\
\hline & E. coli & 12.5 & 12.5 & & & \\
\hline & P. aeruginosa & 25 & 25 & & & \\
\hline & P. vulgaris & 12.5 & 12.5 & & & \\
\hline & C. albicans & 6.25 & 6.25 & & & \\
\hline & C. mandshurica & 3.125 & 3.125 & & & \\
\hline & P. piricola & 1.56 & 1.56 & & & \\
\hline & A. niger & 6.25 & 6.25 & & & \\
\hline
\end{tabular}


Table 1. Cont.

\begin{tabular}{|c|c|c|c|c|c|c|}
\hline $\begin{array}{c}\text { Series/ } \\
\text { Compound }\end{array}$ & Strain & $\mathrm{MIC}, \mathrm{mg} \cdot \mathrm{L}^{-1}$ & $\mathrm{MBC}, \mathrm{mg} \cdot \mathrm{L}^{-1}$ & Method & Notes & Ref. \\
\hline \multirow{10}{*}{24} & S. aureus & 12.5 & 25 & \multirow{10}{*}{ Broth tube dilution } & & \multirow{10}{*}[61]{} \\
\hline & a-H-tococcus & 12.5 & 12.5 & & & \\
\hline & $b$-H-tococcus & 6.25 & 6.25 & & & \\
\hline & E. coli & 25 & 25 & & & \\
\hline & P. aeruginosa & 50 & 50 & & & \\
\hline & P. vulgaris & 25 & 25 & & & \\
\hline & C. albicans & 12.5 & 12.5 & & & \\
\hline & C. mandshurica & 12.5 & 12.5 & & & \\
\hline & P. piricola & 6.25 & 6.25 & & & \\
\hline & A. niger & 12.5 & 12.5 & & & \\
\hline \multirow[b]{2}{*}{25} & S. aureus ATCC $209 \mathrm{p}$ & $12.5 \mu \mathrm{M}$ & & \multirow[b]{2}{*}{ Broth microdilution } & & \multirow[b]{2}{*}[62]{} \\
\hline & B. cereus ATCC 8035 & $401 \mu \mathrm{M}$ & & & & \\
\hline \multirow{3}{*}{27} & S. aureus ATCC $209 \mathrm{p}$ & $6.9 \mu \mathrm{M}$ & & \multirow{3}{*}{ Broth microdilution } & & \multirow{3}{*}[62]{} \\
\hline & B. cereus ATCC 8035 & $28.0 \mu \mathrm{M}$ & & & & \\
\hline & C. albicans $855-653$ & $222 \mu \mathrm{M}$ & & & & \\
\hline 29 & S. aureus & $14.3 \mathrm{~mm}(\mathrm{IZ}, 500 \mathrm{ppm})$ & & Disk diffusion & & [63] \\
\hline
\end{tabular}


Table 1. Cont.

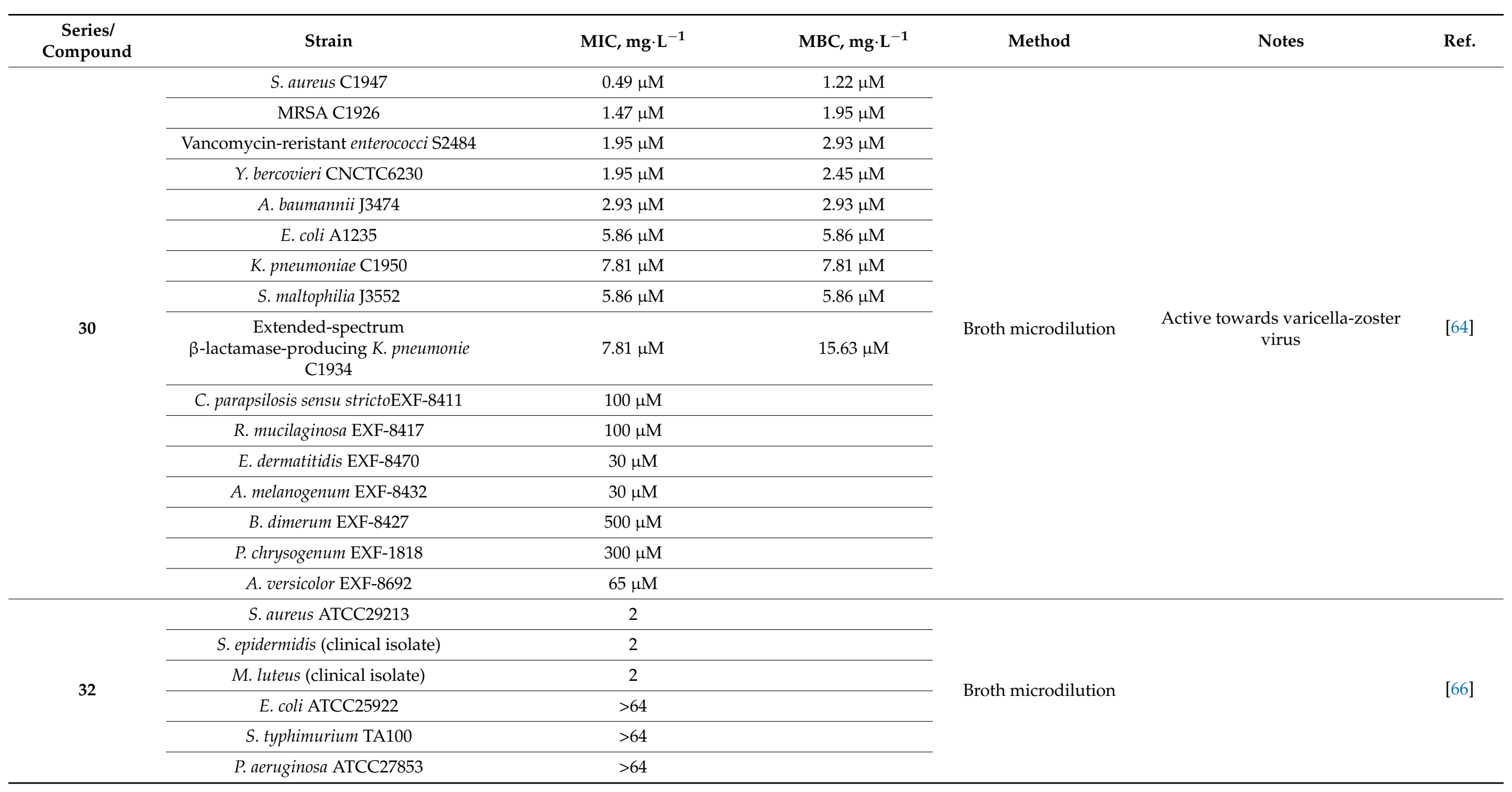


Table 1. Cont.

\begin{tabular}{|c|c|c|c|c|c|c|}
\hline $\begin{array}{c}\text { Series/ } \\
\text { Compound }\end{array}$ & Strain & $\mathrm{MIC}, \mathrm{mg} \cdot \mathrm{L}^{-1}$ & $\mathrm{MBC}, \mathrm{mg} \cdot \mathrm{L}^{-1}$ & Method & Notes & Ref. \\
\hline \multirow{6}{*}{33} & S. aureus ATCC29213 & 4 & & \multirow{6}{*}{ Broth microdilution } & & \multirow{6}{*}[66]{} \\
\hline & S. epidermidis (clinical isolate) & 4 & & & & \\
\hline & M. luteus (clinical isolate) & 2 & & & & \\
\hline & E. coli ATCC25922 & $>64$ & & & & \\
\hline & S. typhimurium TA100 & 4 & & & & \\
\hline & P. aeruginosa ATCC27853 & $>64$ & & & & \\
\hline \multirow{6}{*}{34} & S. aureus ATCC29213 & 0.5 & & \multirow{6}{*}{ Broth microdilution } & & \multirow{6}{*}[66]{} \\
\hline & S. epidermidis (clinical isolate) & 0.5 & & & & \\
\hline & M. luteus (clinical isolate) & 0.5 & & & & \\
\hline & E. coli ATCC25922 & 2 & & & & \\
\hline & S. typhimurium TA100 & 0.5 & & & & \\
\hline & P. aeruginosa ATCC27853 & $>64$ & & & & \\
\hline \multirow{4}{*}{35} & S. epidermidis (clinical isolate) & 2 & & \multirow{4}{*}{ Broth microdilution } & \multirow{4}{*}{$\begin{array}{l}\text { Non-genotoxic and } \\
\text { non-mutagenic }\end{array}$} & \multirow{4}{*}[70]{} \\
\hline & M. luteus (clinical isolate) & 1 & & & & \\
\hline & E. coli ATCC25922 & 8 & & & & \\
\hline & P. aeruginosa ATCC27853 & 8 & & & & \\
\hline \multirow{6}{*}{36} & S. aureus ATCC 29213 & 4 & 8 & \multirow{6}{*}{ Broth microdilution } & \multirow{6}{*}{$\begin{array}{l}\text { Active towards bacterial, fungi } \\
\text { and mixed biofilms }\end{array}$} & \multirow{6}{*}[69]{} \\
\hline & B. subtilis 168 & 4 & 8 & & & \\
\hline & S. epidermidis & 4 & 8 & & & \\
\hline & E. coli MG1655 & 16 & 16 & & & \\
\hline & K. pneumoniae & $>64$ & $>64$ & & & \\
\hline & P. aeruginosa ATCC 27853 & 64 & 64 & & & \\
\hline
\end{tabular}


Table 1. Cont.

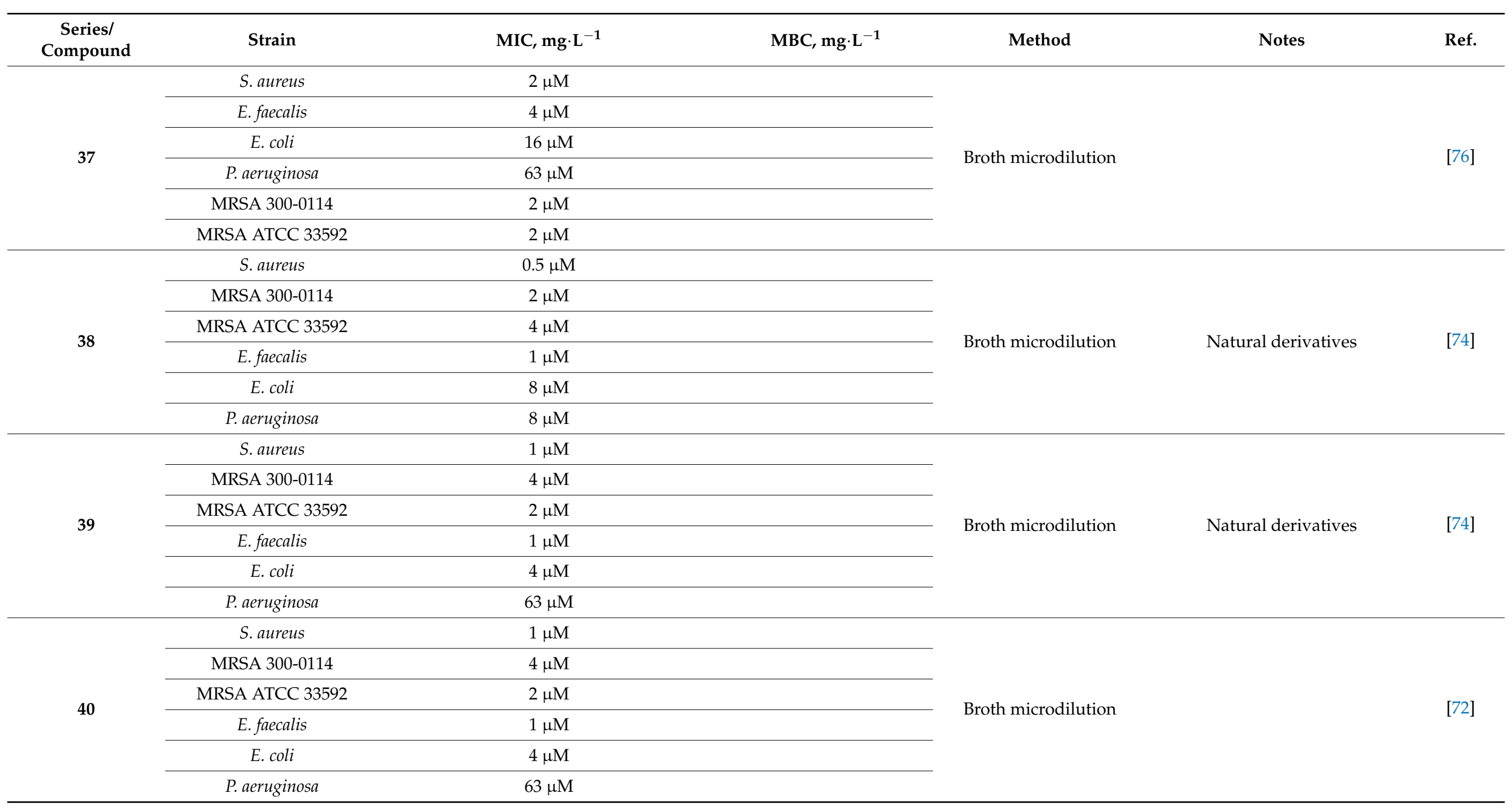


Table 1. Cont.

\begin{tabular}{|c|c|c|c|c|c|c|}
\hline $\begin{array}{c}\text { Series/ } \\
\text { Compound }\end{array}$ & Strain & $\mathrm{MIC}, \mathrm{mg} \cdot \mathrm{L}^{-1}$ & $\mathrm{MBC}, \mathrm{mg} \cdot \mathrm{L}^{-1}$ & Method & Notes & Ref. \\
\hline \multirow{4}{*}{41} & S. aureus SH1000 & $1 \mu \mathrm{M}$ & & \multirow{4}{*}{ Broth microdilution } & & \multirow{4}{*}[75]{} \\
\hline & E. faecalis OG1RF & $16 \mu \mathrm{M}$ & & & & \\
\hline & E. coli MC4100 & $16 \mu \mathrm{M}$ & & & & \\
\hline & P. aeruginosa PAO1-WT & $16 \mu \mathrm{M}$ & & & & \\
\hline
\end{tabular}

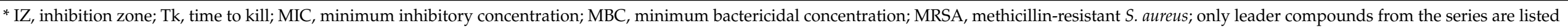
in the table. 


\section{Cations}

Imidazolium<smiles></smiles>

$\left[\mathrm{C}_{\mathrm{n}+1} \operatorname{Mim}\right]$

$\mathrm{n}=1,3,5,7,9,11,13,15$

Pyridinium<smiles>CC(C)(C)[n+]1ccccc1</smiles>

$\left[\mathrm{C}_{\mathrm{n}+1} \mathrm{Py}\right]$ $n=3,5,7,9,11,13,15$<smiles>Cn1cc[n+](CCO)c1</smiles>

[HOC 2 Mim]<smiles></smiles>

$n=5$

\section{Piperidinium}

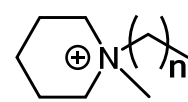

[C ${ }_{12} \mathrm{C}_{1}$ Pip]

$\mathrm{n}=11$

\section{Morpholinium}

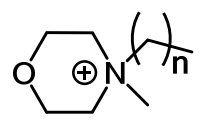

[C ${ }_{12} \mathrm{C}_{1}$ Mor]

$\mathrm{n}=11$

\section{Quaternary ammonium}

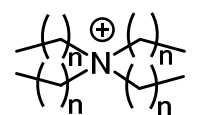

$\left[\mathrm{C}_{\mathrm{n}+1, \mathrm{n}+1, \mathrm{n}+1, \mathrm{n}+1} \mathrm{~N}\right]$ $\mathrm{n}=0,1,7,9,11$, $13,15,17$<smiles>CC1(CO)CCCC[N+]1(C)C</smiles>

$\left[\mathrm{HOC}_{2} \mathrm{C}_{1,1, \mathrm{n}+1} \mathrm{~N}\right]$<smiles>CN1CCC2(CO)CCCC1N2CCO</smiles>

$\left[\left(\mathrm{HOC}_{2}\right)_{2} \mathrm{C}_{\mathrm{n}+1,1} \mathrm{~N}\right]$

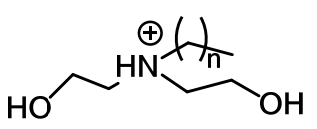

$\left[\left(\mathrm{HOC}_{2}\right)_{2} \mathrm{C}_{14} \mathrm{NH}\right]$

\section{Quaternary phosphonium}

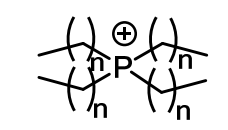

$\left[C_{n+1, n+1, n+1, n+1} P\right]$

$\mathrm{n}=0,5,7,13$

Anions

\section{Hexafluorophosphate}<smiles>FP(F)(F)(F)P(F)(F)(F)F</smiles>

$\left[\mathrm{PF}_{6}\right]$

\section{Tetrafluoroborate}

$\mathrm{F}^{-\mathrm{B}^{-}} \mathrm{F}_{\mathrm{F}}$

$\left[\mathrm{BF}_{4}\right]$

\section{Dicyanamide}<smiles></smiles>

$\left[\mathrm{N}(\mathrm{CN})_{2}\right]$
Thiocyanate

$\mathrm{s} \stackrel{\ominus}{=} \mathrm{N}$

[SCN]
Bis(trifluoromethanesulfonyl)imide<smiles>O=S(=O)(NS(=O)(=O)C(F)(F)F)NS(=O)(=O)C(F)(F)F</smiles>

Hydrogen sulfate<smiles>O=S(=O)([O-])O</smiles>

$\left[\mathrm{HSO}_{4}\right]$<smiles>COS(=O)(=O)[O-]</smiles>

$\left[\mathrm{C}_{1} \mathrm{OSO}_{3}\right]$

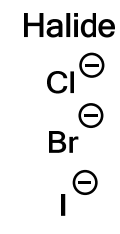

Figure 18. Cations and anions commonly used in ILs with known antimicrobial activity.

Initially, ILs were considered green solvents that could replace traditional toxic organic solvents in various chemical processes [78]. However, when evidence of the high biological activity of various classes of ILs has emerged, these substances have quickly become candidates for new drugs and drug-like molecules. In particular, the antimicrobial 
activity of ILs has attracted much attention, and their possible medical and environmental applications have been proposed $[12,13,79,80]$.

A subclass of ILs with quaternary ammonium cations (which includes several of the above-discussed QACs) has promptly been established as a promising alternative to traditional antimicrobial substances [80]. ILs with other cations have also demonstrated prominent bactericidal and fungicidal activities [12,79]. Some of these ILs (e.g., $\mathrm{N}$-hexadecylpyridinium chloride, or cetylpyridinium chloride, CPC, which is also classified as a QAC) have been extensively used as antiseptics for a long time [81,82]. The first successful results of studies on the antimicrobial activities of various ILs have led to the rapid development of API-ILs (active pharmaceutical ingredient-ionic liquid), that is, known commercial drugs in an ionic liquid form [12,83,84].

An overview of the antimicrobial activities of various members of common IL classes is provided in Table 2 and Table S1. In most cases, there is a direct relation between the length of the alkyl side chain in the cation and the IL antimicrobial activity. ILs with relatively short side chains (ethyl, butyl, hexyl) usually demonstrate weak activity (see Table S1), whereas those with long side chains (dodecyl, tetradecyl, hexadecyl) can be strong inhibitors of some bacterial and fungal species, including biofilmforming and drug-resistant species (see, e.g., entries for $\left[\mathrm{C}_{\mathrm{n}} \mathrm{Mim}\right][\mathrm{A}], \mathrm{n}=12-16$, and [ $\left.\mathrm{C}_{\mathrm{n}} \mathrm{Py}\right], \mathrm{n}=12-16$, in Table 2) [81,85-89]. For instance, 1-dodecyl-3-methylimidazolium bromide ([C $\left.\left.\mathrm{C}_{12} \mathrm{Mim}\right][\mathrm{Br}]\right), \mathrm{N}$-dodecyl- $\mathrm{N}$-methylpyrrolidinium bromide $\left(\left[\mathrm{C}_{12} \mathrm{C}_{1} \mathrm{Pyr}\right][\mathrm{Br}]\right)$, and $\mathrm{N}$-dodecyl- $\mathrm{N}$-methylpiperidinium bromide $\left(\left[\mathrm{C}_{12} \mathrm{C}_{1} \mathrm{Pip}\right][\mathrm{Br}]\right)$ demonstrated both high antimicrobial and low hemolytic activity, thus allowing their successful application in medicinal practice [90,91]. Cholinium-based ILs with long alkyl chains, in particular, $\mathrm{N}$-(2-hydroxyethyl)- $\mathrm{N}, \mathrm{N}$-dimethyl- $\mathrm{N}$-tetradecylammonium bromide, $\mathrm{N}$-(2-hydroxyethyl)$\mathrm{N}, \mathrm{N}$-dimethyl- $\mathrm{N}$-hexadecylammonium bromide, and $\mathrm{N}$-(2-hydroxyethyl)- $\mathrm{N}, \mathrm{N}$-dimethyl$\mathrm{N}$-octadecylammonium bromide, efficiently inhibited the growth of various bacterial strains, including antibiotic-resistant strains (see entries for $\left[\mathrm{HOC}_{2} \mathrm{C}_{1,1, \mathrm{n}} \mathrm{N}\right][\mathrm{Br}], \mathrm{n}=14-18$, in Table 2) [92]. Surface-active cholinium ILs with the dodecylbenzenesulfonate anion demonstrated significant activity against Gram-negative and Gram-positive bacteria, fungi, and single-cell algae; these ILs were proposed to be used as coatings for the prevention of biofilm formation on stone surfaces [93].

It should be noted that the anion can also have a significant impact on the antimicrobial activity. Thus, the antibacterial activity of 1-butyl-3-methylimidazolium ILs with different anions against pathogenic and semipathogenic Gram-negative and Gram-positive bacteria varied significantly depending on the anionic nature [94]. In particular, 1-butyl-3methylimidazolium bis(trifluoromethanesulfonyl)imide $\left(\left[\mathrm{C}_{4} \mathrm{Mim}\right]\left[\mathrm{NTf}_{2}\right]\right)$ demonstrated the highest activity against E. coli (see entries for $\left[\mathrm{C}_{4} \mathrm{Mim}\right][\mathrm{A}]$ in Table 2 and Table S1); however, its anti-adhesive activity was significantly lower than that of several other ILs tested. A different picture was observed in the case of 1-hexyl-3-methylimidazolium $\mathrm{IL}$, among which 1-hexyl-3-methylimidazolium nitrate $\left(\left[\mathrm{C}_{6} \mathrm{Mim}\right]\left[\mathrm{NO}_{3}\right]\right)$ demonstrated the highest activity against $E$. coli and several other microorganisms tested (see entries for [ $\left.\mathrm{C}_{6} \mathrm{Mim}\right][\mathrm{A}]$ in Table S1) [95]. Interestingly, it was demonstrated that for ILs with tris(pentafluoroethyl)trifluorophosphate anions, the antimicrobial activity decreased upon increasing the alkyl side chain length [96].

Of special interest are ILs containing antimicrobial moieties in their anions or cations. The API-IL concept allows simultaneously solving two common issues of traditional drugs: low solubility in aqueous media and tendency to form polymorphs [12]. Examples of bactericidal API-ILs are given in Figure 19, Table 3, and Table S2. Thus, API-ILs bearing ampicillin as their anion in combination with cetylpyridinium or 1-hexadecyl-2,3dimethylimidazolium as their cation demonstrated improved activity against several Gramnegative and Gram-positive bacterial strains, including ampicillin-resistant E. coli strains, compared to the ampicillin sodium salt (see the corresponding entries in Table 3) [82,97]. 


\section{Cations}<smiles>CCCCCC[n+]1cc(C)n(C)c1</smiles><smiles>C[N+](C)(C)Cc1ccccc1</smiles>

[BA]<smiles></smiles>

$\left[\mathrm{C}_{16} \mathrm{Py}\right]$

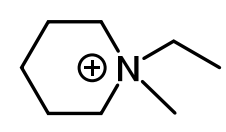

$\left[\mathrm{C}_{2} \mathrm{C}_{1}\right.$ Pip $]$

$n=11,13$<smiles>CCCCCCCCCCCCCCC[N+](C)(CCCCCCCC)CCCCC[N+](C)(CCCCCCCCC)CCCCCCCCC</smiles>
$\left[\mathrm{C}_{1,1,1,16} \mathrm{~N}\right]$<smiles>CCCC[N+](CCCC)(CCCC)CCCC</smiles>
$\left[\mathrm{C}_{8,8,1,1} \mathrm{~N}\right]$ $\left[\mathrm{C}_{8,8,8,1} \mathrm{~N}\right]$<smiles>CCCCCCCCCCC[N+](C)(C)CCCCCCCCCC</smiles><smiles>C[N+](C)(C)CCO</smiles>
[Chol]

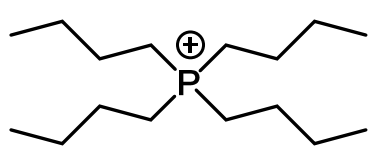

$\left[\mathrm{C}_{4,4,4,4} \mathrm{P}\right]$

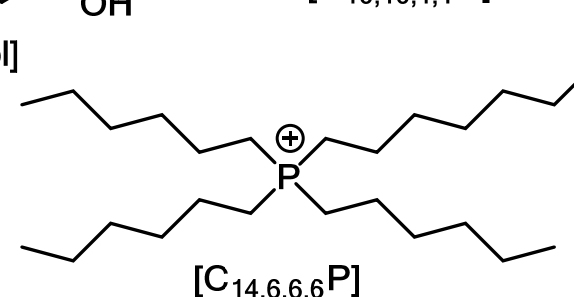

$\left[\mathrm{C}_{14,6,6,6} \mathrm{P}\right]$

\section{Anions}<smiles>CC1(C)S[C@H]2[C@H](NC(=O)[C@@H](N)c3ccccc3)C(=O)N2[C@H]1C(=O)[O-]</smiles><smiles>CCN1C=C(C(=O)[O-])C(=O)C2C=CC(C)=NC21</smiles><smiles>O=C1N[NH+]([O-])S(=O)(=O)c2ccccc21</smiles><smiles>CC1=CC(=O)NS(=O)(=O)O1</smiles>

Figure 19. Cations and anions used in antimicrobial API-ILs. 
Table 2. Antimicrobial activity of common ILs *.

\begin{tabular}{|c|c|c|c|c|c|c|c|}
\hline IL & Acronym & Species & $\mathrm{MIC}, \mu \mathrm{g} \mathrm{mL}^{-1}$ & $\mathrm{MBC}, \mu \mathrm{g} \mathrm{mL}{ }^{-1}$ & Method & Notes & Ref. \\
\hline \multirow{8}{*}{$\begin{array}{l}\text { 1-Ethyl-3- } \\
\text { methylimidazolium } \\
\text { bromide }\end{array}$} & \multirow{8}{*}[\mathrm{C}_{2}\mathrm{Mim}]{$[\mathrm{Br}]$} & E. coli ATCC 25922 & $>5000 \mu \mathrm{M}$ & & \multirow{8}{*}{ Broth microdilution } & \multirow{8}{*}{$\begin{array}{l}\text { E. coli TEM CTX M9, CTX M2, } \\
\text { and AmpC MOX2 are } \\
\text { ampicillin-resistant strains. }\end{array}$} & \multirow{8}{*}{ [82] } \\
\hline & & E. coli TEM CTX M9 & $5000 \mu \mathrm{M}$ & & & & \\
\hline & & E. coli CTX M2 & $>5000 \mu \mathrm{M}$ & & & & \\
\hline & & E. coli AmpC MOX2 & $>5000 \mu \mathrm{M}$ & & & & \\
\hline & & K. pneumoniae (clinical isolate) & $>5000 \mu \mathrm{M}$ & & & & \\
\hline & & S. aureus ATCC 25293 & $50 \mu \mathrm{M}$ & & & & \\
\hline & & S. epidermidis (clinical isolate) & $5000 \mu \mathrm{M}$ & & & & \\
\hline & & E. faecalis (clinical isolate) & $>5000 \mu \mathrm{M}$ & & & & \\
\hline \multirow{7}{*}{$\begin{array}{l}\text { 1-Butyl-3- } \\
\text { methylimidazolium } \\
\text { bis(trifluoromethan- } \\
\text { esulfonyl)imide }\end{array}$} & \multirow{7}{*}[\mathrm{C}_{4}\mathrm{Mim}]{$\left[\mathrm{NTf}_{2}\right]$} & P. aeruginosa PTCC 1310 & 3120 & 3120 & \multirow{7}{*}{$\begin{array}{c}\text { Agar disk } \\
\text { diffusion/agar well } \\
\text { diffusion }\end{array}$} & \multirow{7}{*}{ Anti-adhesive activity ${ }^{a}$} & \multirow{7}{*}{ [94] } \\
\hline & & S. aureus PTCC 1112 & 3120 & 3120 & & & \\
\hline & & E. coli PTCC 1338 & $<40$ & 48 & & & \\
\hline & & B. cereus PTCC 1015 & 3120 & 3120 & & & \\
\hline & & S. typhimurium (wild type) & 390 & 390 & & & \\
\hline & & K. pneumonia PTCC 1290 & 3120 & 3120 & & & \\
\hline & & B. subtilis PTCC 1715 & 3120 & 3120 & & & \\
\hline \multirow{14}{*}{$\begin{array}{l}\text { 1-Octyl-3- } \\
\text { methylimidazolium } \\
\text { bromide }\end{array}$} & \multirow{14}{*}[\mathrm{C}_{8}\mathrm{Mim}]{$[\mathrm{Br}]$} & S. epidermidis ATCC155-1 & $930 \mu \mathrm{M}$ & & \multirow{14}{*}{ Broth microdilution } & \multirow{14}{*}{$\begin{array}{l}\mathrm{R} \text {, resistant at the highest } \\
\text { concentration } \\
\text { tested }(256 \mu \mathrm{g} \mathrm{mL}-1) \text {. }\end{array}$} & \multirow{14}{*}[81,87]{} \\
\hline & & S. aureus ATCC 25178 & $\mathrm{R}$ & & & & \\
\hline & & S. aureus 209 KCTC1916 & 64 & & & & \\
\hline & & S. aureus R209 KCTC1928 & 250 & & & & \\
\hline & & E. coli ATCC 27325 & $\mathrm{R}$ & & & & \\
\hline & & E. coli KCTC1924 & 64 & & & & \\
\hline & & K. pneumonia АТСС 9721 & $\mathrm{R}$ & & & & \\
\hline & & P. aeruginosa ATCC 9721 & $\mathrm{R}$ & & & & \\
\hline & & C. albicans ATCC10231 & $\mathrm{R}$ & & & & \\
\hline & & C. albicans KCTC19401 & 250 & & & & \\
\hline & & B. subtilis ATCC663 & $\mathrm{R}$ & & & & \\
\hline & & B. subtilis KCTC1914 & 500 & & & & \\
\hline & & S. typhimurium KCTC1926 & 500 & & & & \\
\hline & & C. regularis & 500 & & & & \\
\hline
\end{tabular}


Table 2. Cont.

\begin{tabular}{|c|c|c|c|c|c|c|c|}
\hline IL & Acronym & Species & $\mathrm{MIC}, \mu \mathrm{g} \mathrm{mL}^{-1}$ & $\mathrm{MBC}, \mu \mathrm{g} \mathrm{mL^{-1 }}$ & Method & Notes & Ref. \\
\hline \multirow{7}{*}{$\begin{array}{l}\text { 1-Octyl-3- } \\
\text { methylimidazolium } \\
\text { nitrate }\end{array}$} & \multirow{7}{*}[\mathrm{C}_{8}\mathrm{Mim}]{$\left[\mathrm{NO}_{3}\right]$} & S. aureus & 97 & 97 & \multirow{7}{*}{$\begin{array}{c}\text { Agar disk } \\
\text { diffusion/agar well } \\
\text { diffusion }\end{array}$} & \multirow{7}{*}{ Anti-adhesive activity ${ }^{a}$} & \multirow{7}{*}{ [95] } \\
\hline & & K. pneumoniae & 780 & 780 & & & \\
\hline & & S. typhimurium & 780 & 780 & & & \\
\hline & & P. aeruginosa & 1560 & 1560 & & & \\
\hline & & E. coli & 39 & 39 & & & \\
\hline & & B. tequilensis & 19 & 19 & & & \\
\hline & & B. subtilis & 19 & 19 & & & \\
\hline \multirow{21}{*}{$\begin{array}{l}\text { 1-Decyl-3- } \\
\text { methylimidazolium } \\
\text { chloride }\end{array}$} & \multirow{21}{*}[\mathrm{C}_{10}\mathrm{Mim}]{$[\mathrm{Cl}]$} & S. aureus ATCC 29213 & $40 \mu \mathrm{M}(\mathrm{MBEC} 2415 \mu \mathrm{M})$ & $643 \mu \mathrm{M}$ & \multirow{21}{*}{$\begin{array}{l}\text { Broth microdilution, } \\
\text { MBEC assay }\end{array}$} & \multirow{21}{*}{$\begin{array}{l}\text { Deletions } \Delta \mathrm{rfaC}, \Delta \mathrm{rfaL} \text {, and } \\
\Delta \mathrm{rfaG} \text { affect the cell surface } \\
\text { hydrophobicity and } \\
\text { membrane permeability. }\end{array}$} & \multirow{21}{*}[81,85,86]{} \\
\hline & & E-MRSA 15 & $40 \mu \mathrm{M}(\mathrm{MBEC} 1207 \mu \mathrm{M})$ & $321 \mu \mathrm{M}$ & & & \\
\hline & & MRSA (clinical strain 201) & $160 \mu \mathrm{M}(\mathrm{MBEC} 4829 \mu \mathrm{M})$ & $643 \mu \mathrm{M}$ & & & \\
\hline & & S. aureus 209 KCTC1916 & 16 & & & & \\
\hline & & S. aureus R209 KCTC1928 & 32 & & & & \\
\hline & & S. epidermidis ATCC 12228 & $40 \mu \mathrm{M}$ & $644 \mu \mathrm{M}$ & & & \\
\hline & & S. epidermidis ATCC 35984 & $40 \mu \mathrm{M}(\mathrm{MBEC} 4829 \mu \mathrm{M})$ & $160 \mu \mathrm{M}$ & & & \\
\hline & & E. coli NCTC 8196 & $321 \mu \mathrm{M}(\mathrm{MBEC} 9659 \mu \mathrm{M})$ & $1287 \mu \mathrm{M}$ & & & \\
\hline & & E. coli BW25113 (wild-type) & 188.9 & & & & \\
\hline & & E. coli JW3596 $(\Delta \mathrm{rfaC})$ & 100 & & & & \\
\hline & & E. coli JW3597 ( $\Delta \mathrm{rfaL})$ & 155 & & & & \\
\hline & & E. coli JW3606 $(\Delta \mathrm{rfaG})$ & 67.5 & & & & \\
\hline & & P. aeruginosa PA01 & $\begin{array}{c}>1287 \mu \mathrm{M}(\mathrm{MBEC} 2415 \\
\mu \mathrm{M})\end{array}$ & $>1287 \mu \mathrm{M}$ & & & \\
\hline & & K. aerogenes NCTC 7427 & $643 \mu \mathrm{M}(\mathrm{MBEC} 19318 \mu \mathrm{M})$ & $1287 \mu \mathrm{M}$ & & & \\
\hline & & B. cenocepacia $\mathrm{J} 2315$ & $1287 \mu \mathrm{M}(\underset{\mu \mathrm{MBEC}}{\mathrm{M})} 19318$ & $1287 \mu \mathrm{M}$ & & & \\
\hline & & P. mirabilis NCTC 12442 & $1287 \mu \mathrm{M}(\mathrm{MBEC} 9659 \mu \mathrm{M})$ & $1287 \mu \mathrm{M}$ & & & \\
\hline & & C. tropicalis NCTC 7393 & $321 \mu \mathrm{M}(\mathrm{MBEC} 19318 \mu \mathrm{M})$ & $321 \mu \mathrm{M}$ & & & \\
\hline & & B. subtilis KCTC1914 & 125 & & & & \\
\hline & & S. typhimurium КCTC1926 & 125 & & & & \\
\hline & & C. albicans KCTC19401 & 250 & & & & \\
\hline & & C. regularis & 250 & & & & \\
\hline
\end{tabular}


Table 2. Cont.

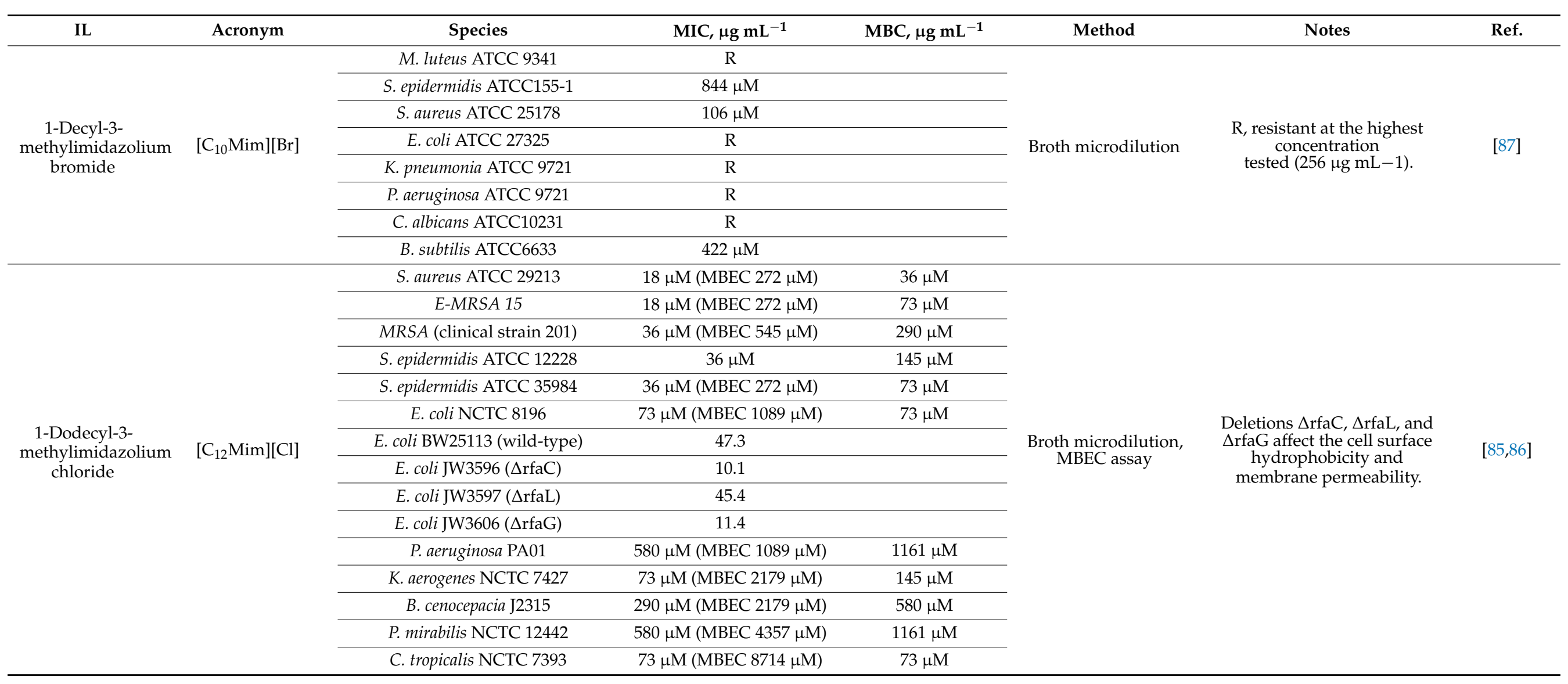


Table 2. Cont.

\begin{tabular}{|c|c|c|c|c|c|c|c|}
\hline IL & Acronym & Species & $\mathrm{MIC}, \mu \mathrm{g} \mathrm{mL}^{-1}$ & $\mathrm{MBC}, \mu \mathrm{g} \mathrm{mL}^{-1}$ & Method & Notes & Ref. \\
\hline \multirow{21}{*}{$\begin{array}{l}\text { 1-Dodecyl-3- } \\
\text { methylimidazolium } \\
\text { bromide }\end{array}$} & \multirow{21}{*}[\mathrm{C}_{12}\mathrm{Mim}]{$[\mathrm{Br}]$} & M. luteus ATCC 9341 & $\mathrm{R}$ & & \multirow{21}{*}{ Broth microdilution } & \multirow{21}{*}{$\begin{array}{l}\mathrm{R} \text {, resistant at the highest } \\
\text { concentration } \\
\text { tested }(256 \mu \mathrm{g} \mathrm{mL}-1) \text {. }\end{array}$} & \multirow{21}{*}[81,87,90,91]{} \\
\hline & & S. epidermidis ATCC155-1 & $193 \mu \mathrm{M}$ & & & & \\
\hline & & S. epidermidis ATCC 35984 & 2.5 & & & & \\
\hline & & S. aureus ATCC 25178 & $97 \mu \mathrm{M}$ & & & & \\
\hline & & S. aureus ATCC 6538 & 2.5 & 40 & & & \\
\hline & & S. aureus 209 KCTC1916 & 4 & & & & \\
\hline & & S. aureus R209 KCTC1928 & 8 & & & & \\
\hline & & E. coli ATCC 27325 & $386 \mu \mathrm{M}$ & & & & \\
\hline & & E. coli ATCC 25922 & 20 & 10 & & & \\
\hline & & E. coli KCTC1924 & 8 & & & & \\
\hline & & K. pneumonia ATCC 9721 & $773 \mu \mathrm{M}$ & & & & \\
\hline & & K. pneumonia АТСС BAA-1705 & 80 & & & & \\
\hline & & P. aeruginosa ATCC 9721 & $\mathrm{R}$ & & & & \\
\hline & & P. aeruginosa ATCC 27853 & 160 & 20 & & & \\
\hline & & C. albicans ATCC10231 & $\mathrm{R}$ & & & & \\
\hline & & B. subtilis KCTC1914 & 8 & & & & \\
\hline & & S. typhimurium KCTC1926 & 32 & & & & \\
\hline & & A. baumannii $\mathrm{AB} 01$ & 80 & & & & \\
\hline & & E. faecalis ATCC 29212 & 5 & 40 & & & \\
\hline & & C. albicans KCTC19401 & 32 & & & & \\
\hline & & C. regularis & 16 & & & & \\
\hline \multirow{2}{*}{$\begin{array}{l}\text { 1-Dodecyl-3- } \\
\text { methylimidazolium } \\
\text { iodide }\end{array}$} & \multirow{2}{*}[\mathrm{C}_{12}\mathrm{Mim}]{$[\mathrm{I}]$} & S. aureus V329 & $0.31 \mu \mathrm{M}$ & $5 \mu \mathrm{M}$ & \multirow{2}{*}{ Broth microdilution } & \multirow{2}{*}{$\begin{array}{l}\text { Potent anti-biofilm activity } \\
\text { (higher against } S \text {. aureus) }\end{array}$} & \multirow{2}{*}{ [98] } \\
\hline & & P. aeruginosa $\mathrm{PAO} 1$ & $125 \mu \mathrm{M}$ & $250 \mu \mathrm{M}$ & & & \\
\hline
\end{tabular}


Table 2. Cont.

\begin{tabular}{|c|c|c|c|c|c|c|c|}
\hline IL & Acronym & Species & $\mathrm{MIC}, \mu \mathrm{g} \mathrm{mL}^{-1}$ & $\mathrm{MBC}, \mu \mathrm{g} \mathrm{mL} \mathrm{L}^{-1}$ & Method & Notes & Ref. \\
\hline \multirow{21}{*}{$\begin{array}{l}\text { 1-Tetradecyl-3- } \\
\text { methylimidazolim } \\
\text { chloride }\end{array}$} & \multirow{21}{*}[\mathrm{C}_{14}\mathrm{Mim}]{$[\mathrm{Cl}]$} & S. aureus ATCC 29213 & $16 \mu \mathrm{M}(\mathrm{MBEC} 124 \mu \mathrm{M})$ & $66 \mu \mathrm{M}$ & \multirow{21}{*}{$\begin{array}{l}\text { Broth microdilution, } \\
\text { MBEC assay }\end{array}$} & \multirow{21}{*}{$\begin{array}{c}\text { Deletions } \Delta \mathrm{rfaC}, \Delta \mathrm{rfaL} \text {, and } \\
\Delta \mathrm{rfaG} \text { affect the cell surface } \\
\text { hydrophobicity and } \\
\text { membrane permeability. }\end{array}$} & \multirow{21}{*}{$81,85,86$} \\
\hline & & E-MRSA 15 & $16 \mu \mathrm{M}(\mathrm{MBEC} 248 \mu \mathrm{M})$ & $66 \mu \mathrm{M}$ & & & \\
\hline & & MRSA (clinical strain 201) & $16 \mu \mathrm{M}(\mathrm{MBEC} 124 \mu \mathrm{M})$ & $66 \mu \mathrm{M}$ & & & \\
\hline & & S. aureus 209 KCTC1916 & 4 & & & & \\
\hline & & S. aureus R209 KCTC1928 & 4 & & & & \\
\hline & & S. epidermidis ATCC 12228 & $7.75 \mu \mathrm{M}$ & $33 \mu \mathrm{M}$ & & & \\
\hline & & S. epidermidis ATCC 35984 & $7.75 \mu \mathrm{M}(\mathrm{MBEC} 124 \mu \mathrm{M})$ & $33 \mu \mathrm{M}$ & & & \\
\hline & & E. coli NCTC 8196 & $33 \mu \mathrm{M}(\mathrm{MBEC} 124 \mu \mathrm{M})$ & $33 \mu \mathrm{M}$ & & & \\
\hline & & E. coli KCTC1924 & 4 & & & & \\
\hline & & E. coli BW25113 (wild-type) & 14.9 & & & & \\
\hline & & E. coli JW3596 $(\Delta \mathrm{rfaC})$ & 2.2 & & & & \\
\hline & & E. coli JW3597 ( $\Delta \mathrm{rfaL})$ & 15.5 & & & & \\
\hline & & E. coli JW3606 ( $\Delta \mathrm{rfaG})$ & 3.3 & & & & \\
\hline & & P. aeruginosa PA01 & $264 \mu \mathrm{M}(\mathrm{MBEC} 496 \mu \mathrm{M})$ & $264 \mu \mathrm{M}$ & & & \\
\hline & & K. aerogenes NCTC 7427 & $33 \mu \mathrm{M}(\mathrm{MBEC} 248 \mu \mathrm{M})$ & $66 \mu \mathrm{M}$ & & & \\
\hline & & P. mirabilis NCTC 12442 & $264 \mu \mathrm{M}(\mathrm{MBEC} 1984 \mu \mathrm{M})$ & $530 \mu \mathrm{M}$ & & & \\
\hline & & C. tropicalis NCTC 7393 & $66 \mu \mathrm{M}(\mathrm{MBEC} 248 \mu \mathrm{M})$ & $132 \mu \mathrm{M}$ & & & \\
\hline & & B. subtilis KCTC1914 & 4 & & & & \\
\hline & & S. typhimurium KCTC1926 & 8 & & & & \\
\hline & & C. albicans KCTC19401 & 8 & & & & \\
\hline & & C. regularis & 8 & & & & \\
\hline
\end{tabular}


Table 2. Cont.

\begin{tabular}{|c|c|c|c|c|c|c|c|}
\hline IL & Acronym & Species & $\mathrm{MIC}, \mu \mathrm{g} \mathrm{mL}^{-1}$ & $\mathrm{MBC}, \mu \mathrm{g} \mathrm{mL} \mathrm{L}^{-1}$ & Method & Notes & Ref. \\
\hline \multirow{15}{*}{$\begin{array}{l}\text { 1-Tetradecyl-3- } \\
\text { methylimidazolim } \\
\text { bromide }\end{array}$} & \multirow{15}{*}[\mathrm{C}_{14}\mathrm{Mim}]{$[\mathrm{Br}]$} & M. luteus ATCC 9341 & $178 \mu \mathrm{M}$ & & \multirow{15}{*}{ Broth microdilution } & & \multirow{15}{*}[81,87]{} \\
\hline & & S. epidermidis ATCC155-1 & $6 \mu \mathrm{M}$ & & & & \\
\hline & & S. aureus ATCC 25178 & $45 \mu \mathrm{M}$ & & & & \\
\hline & & S. aureus 209 KCTC1916 & 4 & & & & \\
\hline & & S. aureus R209 KCTC1928 & 4 & & & & \\
\hline & & E. coli ATCC 27325 & $356 \mu \mathrm{M}$ & & & & \\
\hline & & E. coli KCTC1924 & 4 & & & & \\
\hline & & K. pneumonia ATCC 9721 & $356 \mu \mathrm{M}$ & & & & \\
\hline & & P. aeruginosa ATCC 9721 & $356 \mu \mathrm{M}$ & & & & \\
\hline & & C. albicans ATCC10231 & $178 \mu \mathrm{M}$ & & & & \\
\hline & & B. subtilis ATCC6633 & $6 \mu \mathrm{M}$ & & & & \\
\hline & & B. subtilis КСТC1914 & 4 & & & & \\
\hline & & S. typhimurium KCTC1926 & 8 & & & & \\
\hline & & C. albicans KCTC19401 & 8 & & & & \\
\hline & & C. regularis & 16 & & & & \\
\hline \multirow{9}{*}{$\begin{array}{l}\text { 1-Hexadecyl-3- } \\
\text { methylimidazolim } \\
\text { chloride }\end{array}$} & \multirow{9}{*}[\mathrm{C}_{16}\mathrm{Mim}]{$[\mathrm{Cl}]$} & E. coli JW3596 $(\Delta \mathrm{rfaC})$ & 3.5 & & \multirow{9}{*}{ Broth microdilution } & \multirow{9}{*}{$\begin{array}{l}\text { The clinical isolates } 72 \mathrm{~A}, 72 \mathrm{P}, \\
\text { and } 94 \mathrm{P} \text { are resistant to } \\
\text { fluconazole, amphotericin } \mathrm{B}, \\
\text { voriconazole and } \\
\text { anidulafungin. } \\
\text { Deletions } \Delta \mathrm{rfaC}, \Delta \mathrm{rfaL} \text {, and } \\
\Delta \mathrm{rfaG} \text { affect the cell surface } \\
\text { hydrophobicity and } \\
\text { membrane permeability. }\end{array}$} & \multirow{9}{*}[86,88]{} \\
\hline & & E. coli JW3597 $(\Delta \mathrm{rfaL})$ & 8.2 & & & & \\
\hline & & E. coli JW3606 ( $\Delta \mathrm{rfaG})$ & 3 & & & & \\
\hline & & C. tropicalis 17A & 0.014 (MBEC 0.028) & & & & \\
\hline & & C. tropicalis 57A & 0.014 (MBEC 0.056) & & & & \\
\hline & & C. tropicalis $72 \mathrm{~A}$ & 0.014 (MBEC 0.056) & & & & \\
\hline & & C. tropicalis $72 \mathrm{P}$ & 0.014 (MBEC 0.056) & & & & \\
\hline & & C. tropicalis $94 \mathrm{P}$ & 0.014 (MBEC 0.225) & & & & \\
\hline & & C. tropicalis $102 \mathrm{~A}$ & 0.014 (MBEC 0.056) & & & & \\
\hline
\end{tabular}


Table 2. Cont.

\begin{tabular}{|c|c|c|c|c|c|c|c|}
\hline IL & Acronym & Species & $\mathrm{MIC}, \mu \mathrm{g} \mathrm{mL}^{-1}$ & $\mathrm{MBC}, \mu \mathrm{g} \mathrm{mL} \mathrm{L}^{-1}$ & Method & Notes & Ref. \\
\hline \multirow{10}{*}{$\begin{array}{l}\text { 1-Hexadecyl-3- } \\
\text { methylimidazolim } \\
\text { bromide }\end{array}$} & \multirow{10}{*}[\mathrm{C}_{16}\mathrm{Mim}]{$[\mathrm{Br}]$} & S. aureus 209 KCTC1916 & 8 & & \multirow{10}{*}{ Broth microdilution } & & \multirow{10}{*}[81,97]{} \\
\hline & & S. aureus R209 KCTC1928 & 4 & & & & \\
\hline & & S. aureus ATCC 6538 & $15 \mu \mathrm{M}$ & & & & \\
\hline & & E. coli KCTC1924 & 8 & & & & \\
\hline & & E. coli O157:H7 ATCC 43895 & $10 \mu \mathrm{M}$ & & & & \\
\hline & & B. subtilis KCTC1914 & 4 & & & & \\
\hline & & S. typhimurium КСТС1926 & 4 & & & & \\
\hline & & K. pneumonia ATCC 4352 & $15 \mu \mathrm{M}$ & & & & \\
\hline & & C. albicans KCTC19401 & 8 & & & & \\
\hline & & C. regularis & 8 & & & & \\
\hline \multirow{4}{*}{$\begin{array}{c}\text { 1-Hexyl-2,3- } \\
\text { dimethylimidazolium } \\
\text { bromide }\end{array}$} & \multirow{4}{*}[\mathrm{C}_{6}\mathrm{MMim}]{$[\mathrm{Br}]$} & S. aureus ATCC 6538 & $23 \mu \mathrm{M}$ & & \multirow{4}{*}{ Broth microdilution } & & \multirow{4}{*}[97]{} \\
\hline & & E. coli O157:H7 ATCC 43895 & $12 \mu \mathrm{M}$ & & & & \\
\hline & & E. faecium ATCC 49474 & $9 \mu \mathrm{M}$ & & & & \\
\hline & & K. pneumonia ATCC 4352 & $15 \mu \mathrm{M}$ & & & & \\
\hline \multirow{6}{*}{$\begin{array}{l}N \text {-Dodecy- } \\
\text { lpyridinium } \\
\text { bromide }\end{array}$} & \multirow{6}{*}[\mathrm{C}_{12}\mathrm{Py}]{$[\mathrm{Br}]$} & S. aureus ATCC 25178 & $195 \mu \mathrm{M}$ & & \multirow{6}{*}{ Broth microdilution } & \multirow{6}{*}{$\begin{array}{l}\mathrm{R} \text {, resistant at the highest } \\
\text { concentration } \\
\text { tested }(256 \mu \mathrm{g} \mathrm{mL}-1) \text {. }\end{array}$} & \multirow{6}{*}{ [87] } \\
\hline & & E. coli ATCC 27325 & $97 \mu \mathrm{M}$ & & & & \\
\hline & & K. pneumonia ATCC 9721 & $780 \mu \mathrm{M}$ & & & & \\
\hline & & P. aeruginosa ATCC 9721 & $780 \mu \mathrm{M}$ & & & & \\
\hline & & C. albicans ATCC10231 & $\mathrm{R}$ & & & & \\
\hline & & B. subtilis ATCC6633 & $24 \mu \mathrm{M}$ & & & & \\
\hline \multirow{8}{*}{$\begin{array}{l}N \text {-Tetradecy- } \\
\text { lpyridinium } \\
\text { bromide }\end{array}$} & \multirow{8}{*}[\mathrm{C}_{14}\mathrm{Py}]{$[\mathrm{Br}]$} & M. luteus ATCC 9341 & $90 \mu \mathrm{M}$ & & \multirow{8}{*}{ Broth microdilution } & & \multirow{8}{*}{ [87] } \\
\hline & & S. epidermidis ATCC155-1 & $6 \mu \mathrm{M}$ & & & & \\
\hline & & S. aureus ATCC 25178 & $22 \mu \mathrm{M}$ & & & & \\
\hline & & E. coli ATCC 27325 & $45 \mu \mathrm{M}$ & & & & \\
\hline & & K. pneumonia ATCC 9721 & $359 \mu \mathrm{M}$ & & & & \\
\hline & & P. aeruginosa ATCC 9721 & $359 \mu \mathrm{M}$ & & & & \\
\hline & & C. albicans ATCC10231 & $359 \mu \mathrm{M}$ & & & & \\
\hline & & B. subtilis ATCC6633 & $6 \mu \mathrm{M}$ & & & & \\
\hline
\end{tabular}


Table 2. Cont

\begin{tabular}{|c|c|c|c|c|c|c|c|}
\hline IL & Acronym & Species & MIC, $\mu \mathrm{g} \mathrm{mL}^{-1}$ & $\mathrm{MBC}, \mu \mathrm{g} \mathrm{mL} \mathrm{L}^{-1}$ & Method & Notes & Ref. \\
\hline \multirow{10}{*}{$\begin{array}{l}N \text {-Hexadecy- } \\
\text { lpyridinium } \\
\text { chloride }\end{array}$} & \multirow{10}{*}[\mathrm{C}_{16}\mathrm{Py}]{$[\mathrm{Cl}]$} & E. coli ATCC 25922 & $500 \mu \mathrm{M}$ & & \multirow{10}{*}{ Broth microdilution } & \multirow{10}{*}{$\begin{array}{l}\text { E. coli TEM CTX M9, CTX M2, } \\
\text { and AmpC MOX2 are } \\
\text { ampicillin-resistant strains. }\end{array}$} & \multirow{10}{*}[81,82]{} \\
\hline & & E. coli TEM CTX M9 & $500 \mu \mathrm{M}$ & & & & \\
\hline & & E. coli CTX M2 & $>5000 \mu \mathrm{M}$ & & & & \\
\hline & & E. coli AmpC MOX2 & $>5000 \mu \mathrm{M}$ & & & & \\
\hline & & K. pneumoniae (clinical isolate) & $2500 \mu \mathrm{M}$ & & & & \\
\hline & & S. aureus ATCC 25293 & $500 \mu \mathrm{M}$ & & & & \\
\hline & & S. aureus 209 KCTC1916 & 8 & & & & \\
\hline & & S. epidermidis (clinical isolate) & $2500 \mu \mathrm{M}$ & & & & \\
\hline & & E. faecalis (clinical isolate) & $500 \mu \mathrm{M}$ & & & & \\
\hline & & B. subtilis KCTC1914 & 8 & & & & \\
\hline \multirow{4}{*}{$\begin{array}{l}N \text {-Hexadecy- } \\
\text { lpyridinium } \\
\text { bromide }\end{array}$} & \multirow{4}{*}[\mathrm{C}_{16}\mathrm{Py}]{$[\mathrm{Br}]$} & S. aureus ATCC 6538 & $15 \mu \mathrm{M}$ & & \multirow{4}{*}{ Broth microdilution } & & \multirow{4}{*}{ [97] } \\
\hline & & E. coli O157:H7 ATCC 43895 & $13 \mu \mathrm{M}$ & & & & \\
\hline & & E. faecium ATCC 49474 & $2 \mu \mathrm{M}$ & & & & \\
\hline & & K. pneumonia ATCC 4352 & $13 \mu \mathrm{M}$ & & & & \\
\hline \multirow{8}{*}{$\begin{array}{l}N \text {-Dodecyl- } N \text { - } \\
\text { methylpyrrolidinium } \\
\text { bromide }\end{array}$} & \multirow{8}{*}[\mathrm{C}_{12}\mathrm{C}_{1}\mathrm{Pyr}]{$[\mathrm{Br}]$} & S. aureus & $15 \mu \mathrm{M}$ & & \multirow{8}{*}{ Broth microdilution } & & \multirow{8}{*}{ [89-91] } \\
\hline & & S. aureus ATCC 6538 & 10 & 80 & & & \\
\hline & & E. coli & $20 \mu \mathrm{M}$ & & & & \\
\hline & & E. coli ATCC 25922 & 80 & 20 & & & \\
\hline & & P. aeruginosa ATCC 27853 & 320 & 80 & & & \\
\hline & & K. pneumonia ATCC BAA-1705 & 160 & & & & \\
\hline & & A. baumannii $\mathrm{AB} 01$ & 80 & & & & \\
\hline & & E. faecalis ATCC 29212 & 20 & 40 & & & \\
\hline \multirow{4}{*}{$\begin{array}{l}N \text {-Dodecyl- } N \text { - } \\
\text { hydroxyethy- } \\
\text { lpyrrolidinium } \\
\text { chloride }\end{array}$} & \multirow{4}{*}[\mathrm{C}_{12}\mathrm{HOC}_{2}\mathrm{Pyr}]{$[\mathrm{Cl}]$} & E. coli KCTC1924 & 8 & & \multirow{4}{*}{ Broth microdilution } & & \multirow{4}{*}{ [81] } \\
\hline & & S. typhimurium KCTC1926 & 16 & & & & \\
\hline & & B. subtilis KCTC1914 & 4 & & & & \\
\hline & & C. regularis & 8 & & & & \\
\hline
\end{tabular}


Table 2. Cont.

\begin{tabular}{|c|c|c|c|c|c|c|c|}
\hline IL & Acronym & Species & $\mathrm{MIC}, \mu \mathrm{g} \mathrm{mL} \mathrm{L}^{-1}$ & $\mathrm{MBC}, \mu \mathrm{g} \mathrm{mL}^{-1}$ & Method & Notes & Ref. \\
\hline \multirow{7}{*}{$\begin{array}{c}N \text {-Dodecyl- } N- \\
\text { methylpiperidinium } \\
\text { bromide }\end{array}$} & \multirow{7}{*}[\mathrm{C}_{12}\mathrm{C}_{1}\mathrm{Pip}]{$[\mathrm{Br}]$} & S. epidermidis ATCC 35984 & 5 & & \multirow{7}{*}{ Broth microdilution } & & \multirow{7}{*}{90,91} \\
\hline & & S. aureus ATCC 6538 & 5 & 80 & & & \\
\hline & & E. coli ATCC 25922 & 40 & 20 & & & \\
\hline & & P. aeruginosa ATCC 27853 & 320 & 80 & & & \\
\hline & & K. pneumonia ATCC BAA-1705 & 160 & & & & \\
\hline & & A. baumannii AB01 & 320 & & & & \\
\hline & & E. faecalis ATCC 29212 & 10 & 40 & & & \\
\hline \multirow{4}{*}{$\begin{array}{l}N \text {-Dodecyl- } N \text { - } \\
\text { methylmorpholinium } \\
\text { bromide }\end{array}$} & \multirow{4}{*}[\mathrm{C}_{12}\mathrm{C}_{1}\mathrm{Mor}]{$[\mathrm{Br}]$} & S. aureus ATCC 6538 & 20 & & \multirow{4}{*}{ Broth microdilution } & & \multirow{4}{*}[90]{} \\
\hline & & E. coli ATCC 25922 & 156.2 & & & & \\
\hline & & P. aeruginosa ATCC 27853 & 312.5 & & & & \\
\hline & & E. faecalis ATCC 29212 & 40 & & & & \\
\hline \multirow{3}{*}{$\begin{array}{l}\text { Dioctyldimethylamm- } \\
\text { onium } \\
\text { chloride }\end{array}$} & \multirow{3}{*}[\mathrm{C}_{8,8,1,1}\mathrm{N}]{$[\mathrm{Cl}]$} & E. coli BW25113 (wild-type) & 104.2 & & \multirow{3}{*}{ Broth microdilution } & \multirow{3}{*}{$\begin{array}{c}\text { Deletions } \Delta \mathrm{rfaC}, \Delta \mathrm{rfaL}, \text { and } \\
\Delta \mathrm{rfaG} \text { affect the cell surface } \\
\text { hydrophobicity and membrane } \\
\text { permeability. }\end{array}$} & \multirow{3}{*}{ [86] } \\
\hline & & E. coli JW3596 $(\Delta \mathrm{rfaC})$ & 20.8 & & & & \\
\hline & & E. coli JW3597 $(\Delta \mathrm{rfaL})$ & 91.7 & & & & \\
\hline \multirow{4}{*}{$\begin{array}{l}\text { Trioctylmethylamm- } \\
\text { onium chloride }\end{array}$} & \multirow{4}{*}[\mathrm{C}_{8,8,8,1}\mathrm{N}]{$[\mathrm{Cl}]$} & E. coli BW25113 (wild-type) & 6.8 & & \multirow{4}{*}{ Broth microdilution } & \multirow{4}{*}{$\begin{array}{l}\text { Deletions } \Delta \mathrm{rfaC}, \Delta \mathrm{rfaL} \text {, and } \\
\Delta \mathrm{rfaG} \text { affect the cell surface } \\
\text { hydrophobicity and membrane } \\
\text { permeability. }\end{array}$} & \multirow{4}{*}{ [86] } \\
\hline & & E. coli JW3596 $(\Delta \mathrm{rfaC})$ & 1.7 & & & & \\
\hline & & E. coli JW3597 ( $\Delta \mathrm{rfaL})$ & 6.9 & & & & \\
\hline & & E. coli JW3606 ( $\Delta \mathrm{rfaG})$ & 2.5 & & & & \\
\hline \multirow{4}{*}{$\begin{array}{l}\text { Trimethyldecylamm- } \\
\text { onium chloride }\end{array}$} & \multirow{4}{*}[\mathrm{C}_{1,1,1,10}\mathrm{N}]{$[\mathrm{Cl}]$} & E. coli BW25113 (wild-type) & 119.4 & & \multirow{4}{*}{ Broth microdilution } & \multirow{4}{*}{$\begin{array}{l}\text { Deletions } \Delta \mathrm{rfaC}, \Delta \mathrm{rfaL} \text {, and } \\
\Delta \mathrm{rfaG} \text { affect the cell surface } \\
\text { hydrophobicity and membrane } \\
\text { permeability. }\end{array}$} & \multirow{4}{*}{ [86] } \\
\hline & & E. coli JW3596 $(\Delta \mathrm{rfaC})$ & 83 & & & & \\
\hline & & E. coli JW3597 ( $\Delta \mathrm{rfaL})$ & 130 & & & & \\
\hline & & E. coli JW3606 $(\Delta \mathrm{rfaG})$ & 80 & & & & \\
\hline \multirow{4}{*}{$\begin{array}{l}\text { Trimethylhexadecylamm- } \\
\text { onium chloride }\end{array}$} & \multirow{4}{*}[\mathrm{C}_{1,1,1,16}\mathrm{N}]{$[\mathrm{Cl}]$} & E. coli BW25113 (wild-type) & 13.1 & & \multirow{4}{*}{ Broth microdilution } & \multirow{4}{*}{$\begin{array}{c}\text { Deletions } \Delta \mathrm{rfaC}, \Delta \mathrm{rfaL} \text {, and } \\
\Delta \mathrm{rfaG} \text { affect the cell surface } \\
\text { hydrophobicity and membrane } \\
\text { permeability. }\end{array}$} & \multirow{4}{*}{ [86] } \\
\hline & & E. coli JW3596 ( $\triangle \mathrm{rfaC})$ & 2.8 & & & & \\
\hline & & E. coli JW3597 $(\Delta \mathrm{rfaL})$ & 13 & & & & \\
\hline & & E. coli JW3606 ( $\Delta \mathrm{rfaG})$ & 3.3 & & & & \\
\hline
\end{tabular}


Table 2. Cont.

\begin{tabular}{|c|c|c|c|c|c|c|c|}
\hline IL & Acronym & Species & $\mathrm{MIC}, \mu \mathrm{g} \mathrm{mL}{ }^{-1}$ & $\mathrm{MBC}, \mu \mathrm{g} \mathrm{mL}^{-1}$ & Method & Notes & Ref. \\
\hline \multirow{2}{*}{$\begin{array}{l}\text { Trimethylhexadecy- } \\
\text { lammonium bromide } \\
\text { (cetyltrimethylammo- } \\
\text { nium bromide) }\end{array}$} & \multirow[b]{2}{*}[\mathrm{C}_{1,1,1,16}\mathrm{N}]{$[\mathrm{Br}](\mathrm{CTAB})$} & S. aureus V329 & $0.31 \mu \mathrm{M}$ & $5 \mu \mathrm{M}$ & \multirow[b]{2}{*}{ Broth microdilution } & \multirow[b]{2}{*}{$\begin{array}{l}\text { Potent anti-biofilm activity } \\
\text { against S. aureus }\end{array}$} & \multirow[b]{2}{*}{ [98] } \\
\hline & & P. aeruginosa PAO1 & $125 \mu \mathrm{M}$ & $250 \mu \mathrm{M}$ & & & \\
\hline \multirow{10}{*}{$\begin{array}{l}\text { Dimethyldodecyl(2- } \\
\text { hydroxyethyl)ammon- } \\
\text { ium bromide }\end{array}$} & \multirow{10}{*}[\mathrm{HOC}_{2}\mathrm{C}_{1,1,12}\mathrm{N}]{$[\mathrm{Br}]$} & B. subtilis ATCC 6633 & 15.62 & & \multirow{10}{*}{ Broth microdilution } & & \multirow{10}{*}{ [92] } \\
\hline & & M. smegmatis ATCC 607 & 15.62 & & & & \\
\hline & & K. pneumonia ATCC 9997 & N.T. & & & & \\
\hline & & E. faecalis ATCC 29212 & N.T. & & & & \\
\hline & & S. aureus & 31.25 & & & & \\
\hline & & MRSA CIP 106760 & 62.5 & & & & \\
\hline & & E. coli ATCC 25922 & 62.5 & & & & \\
\hline & & P. aeruginosa ATCC 27853 & 250 & & & & \\
\hline & & C. albicans ATCC 10231 & 62.5 & & & & \\
\hline & & S. cerevisiae ATCC 2601 & 7.81 & & & & \\
\hline \multirow{10}{*}{$\begin{array}{l}\text { Dimethyltetradecyl(2- } \\
\text { hydroxyethyl)ammon- } \\
\text { ium bromide }\end{array}$} & \multirow{10}{*}[\mathrm{HOC}_{2}\mathrm{C}_{1,1,14}\mathrm{N}]{$[\mathrm{Br}]$} & B. subtilis ATCC 6633 & 0.98 & & \multirow{10}{*}{ Broth microdilution } & & \multirow{10}{*}{ [92] } \\
\hline & & M. smegmatis ATCC 607 & 1.95 & & & & \\
\hline & & E. faecalis ATCC 29212 & 1.95 & & & & \\
\hline & & VRE ATCC 51299 & 1.95 & & & & \\
\hline & & S. aureus & 7.81 & & & & \\
\hline & & MRSA CIP 106760 & 15.62 & & & & \\
\hline & & E. coli ATCC 25922 & 15.62 & & & & \\
\hline & & P. aeruginosa ATCC 27853 & 125 & & & & \\
\hline & & C. albicans ATCC 10231 & 31.25 & & & & \\
\hline & & S. cerevisiae ATCC 2601 & 1.95 & & & & \\
\hline
\end{tabular}


Table 2. Cont.

\begin{tabular}{|c|c|c|c|c|c|c|c|}
\hline IL & Acronym & Species & $\mathrm{MIC}, \mu \mathrm{g} \mathrm{mL} \mathrm{L}^{-1}$ & $\mathrm{MBC}, \mu \mathrm{g} \mathrm{mL} \mathrm{L}^{-1}$ & Method & Notes & Ref. \\
\hline \multirow{10}{*}{$\begin{array}{l}\text { Dimethylhexadecyl(2- } \\
\text { hydroxyethyl)ammonium } \\
\text { bromide }\end{array}$} & \multirow{10}{*}[\mathrm{HOC}_{2}\mathrm{C}_{1,1,16}\mathrm{N}]{$[\mathrm{Br}]$} & B. subtilis ATCC 6633 & $<0.49$ & & \multirow{10}{*}{ Broth microdilution } & & \multirow{10}{*}{ [92] } \\
\hline & & M. smegmatis ATCC 607 & 3.91 & & & & \\
\hline & & K. pneumonia АТСС 9997 & 0.98 & & & & \\
\hline & & E. faecalis ATCC 29212 & 0.98 & & & & \\
\hline & & VRE ATCC 51299 & 0.98 & & & & \\
\hline & & S. aureus & 1.95 & & & & \\
\hline & & MRSA CIP 106760 & 3.91 & & & & \\
\hline & & P. aeruginosa ATCC 27853 & 250 & & & & \\
\hline & & C. albicans ATCC 10231 & 3.91 & & & & \\
\hline & & S. cerevisiae ATCC 2601 & 1.95 & & & & \\
\hline \multirow{9}{*}{$\begin{array}{l}\text { Dimethyloctadecyl(2- } \\
\text { hydroxyethyl)ammonium } \\
\text { bromide }\end{array}$} & \multirow{9}{*}[\mathrm{HOC}_{2}\mathrm{C}_{1,1,18}\mathrm{N}]{$[\mathrm{Br}]$} & B. subtilis ATCC 6633 & 1.95 & & \multirow{9}{*}{ Broth microdilution } & & \multirow{9}{*}{ [92] } \\
\hline & & M. smegmatis ATCC 607 & 3.91 & & & & \\
\hline & & K. pneumonia АТСС 9997 & 1.95 & & & & \\
\hline & & E. faecalis ATCC 29212 & 1.95 & & & & \\
\hline & & MRSA CIP 106760 & 0.98 & & & & \\
\hline & & E. coli ATCC 25922 & 31.25 & & & & \\
\hline & & P. aeruginosa ATCC 27853 & 125 & & & & \\
\hline & & C. albicans ATCC 10231 & $<0.48$ & & & & \\
\hline & & S. cerevisiae ATCC 2601 & $<0.48$ & & & & \\
\hline
\end{tabular}


Table 2. Cont.

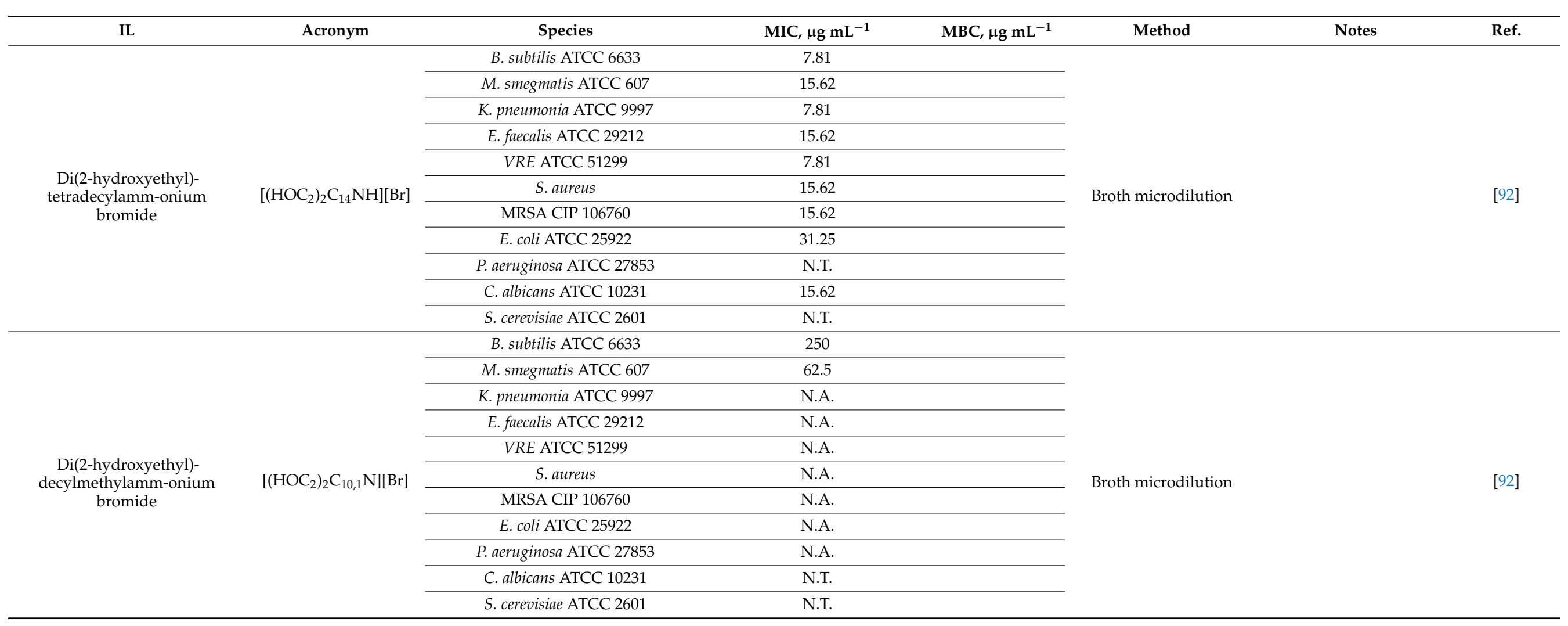


Table 2. Cont.

\begin{tabular}{|c|c|c|c|c|c|c|c|}
\hline IL & Acronym & Species & $\mathrm{MIC}, \mu \mathrm{g} \mathrm{mL}^{-1}$ & $\mathrm{MBC}, \mu \mathrm{g} \mathrm{mL} \mathrm{L}^{-1}$ & Method & Notes & Ref. \\
\hline \multirow{10}{*}{$\begin{array}{l}\text { Di(2-hydroxyethyl)- } \\
\text { dodecylmethylamm- } \\
\text { onium bromide }\end{array}$} & \multirow{10}{*}[(\mathrm{HOC}_{2})_{2}\mathrm{C}_{12,1}\mathrm{N}]{$[\mathrm{Br}]$} & B. subtilis ATCC 6633 & 31.25 & & \multirow{10}{*}{ Broth microdilution } & & \multirow{10}{*}{ [92] } \\
\hline & & M. smegmatis ATCC 607 & $<7.82$ & & & & \\
\hline & & K. pneumonia ATCC 9997 & 62.5 & & & & \\
\hline & & E. faecalis ATCC 29212 & 62.25 & & & & \\
\hline & & VRE ATCC 51299 & 62.5 & & & & \\
\hline & & S. aureus & 31.25 & & & & \\
\hline & & MRSA CIP 106760 & 62.5 & & & & \\
\hline & & P. aeruginosa ATCC 27853 & 250 & & & & \\
\hline & & C. albicans ATCC 10231 & 250 & & & & \\
\hline & & S. cerevisiae ATCC 2601 & 31.25 & & & & \\
\hline \multirow{9}{*}{$\begin{array}{l}\text { Di(2-hydroxyethyl)- } \\
\text { tetradecylmethylamm- } \\
\text { onium bromide }\end{array}$} & \multirow{9}{*}[(\mathrm{HOC}_{2})_{2}\mathrm{C}_{14,1}\mathrm{N}]{$[\mathrm{Br}]$} & B. subtilis ATCC 6633 & 1.95 & & \multirow{9}{*}{ Broth microdilution } & & \multirow{9}{*}{ [92] } \\
\hline & & M. smegmatis ATCC 607 & 1.95 & & & & \\
\hline & & K. pneumonia ATCC 9997 & 7.82 & & & & \\
\hline & & E. faecalis ATCC 29212 & N.T. & & & & \\
\hline & & MRSA CIP 106760 & 1.95 & & & & \\
\hline & & E. coli ATCC 25922 & 15.62 & & & & \\
\hline & & P. aeruginosa ATCC 27853 & 62.5 & & & & \\
\hline & & C. albicans ATCC 10231 & 31.25 & & & & \\
\hline & & S. cerevisiae ATCC 2601 & 1.95 & & & & \\
\hline \multirow{4}{*}{$\begin{array}{l}\text { Trioctylmethylphos- } \\
\text { phonium chloride }\end{array}$} & \multirow{4}{*}[\mathrm{C}_{8,8,8,8,1}\mathrm{P}]{$[\mathrm{Cl}]$} & E. coli BW25113 (wild-type) & 6.8 & & \multirow{4}{*}{ Broth microdilution } & \multirow{4}{*}{$\begin{array}{c}\text { Deletions } \Delta \mathrm{rfaC}, \Delta \mathrm{rfaL} \text {, and } \\
\Delta \mathrm{rfaG} \text { affect the cell surface } \\
\text { hydrophobicity and membrane } \\
\text { permeability. }\end{array}$} & \multirow{4}{*}{ [86] } \\
\hline & & E. coli JW3596 $(\Delta \mathrm{rfaC})$ & 2.2 & & & & \\
\hline & & E. coli JW3597 ( $(\mathrm{rfaL})$ & 5.6 & & & & \\
\hline & & E. coli JW3606 $(\Delta \mathrm{rfaG})$ & 2.8 & & & & \\
\hline
\end{tabular}


Table 2. Cont.

\begin{tabular}{|c|c|c|c|c|c|c|c|}
\hline IL & Acronym & Species & $\mathrm{MIC}, \mu \mathrm{g} \mathrm{mL}^{-1}$ & $\mathrm{MBC}, \mu \mathrm{g} \mathrm{mL}^{-1}$ & Method & Notes & Ref. \\
\hline \multirow{8}{*}{$\begin{array}{l}\text { Trihexyltetradecylphos- } \\
\text { phonium chloride }\end{array}$} & \multirow{8}{*}[\mathrm{C}_{6,6,6,14}\mathrm{P}]{$[\mathrm{Cl}]$} & L. monocytogenes ATCC13932 & 5.7 & & \multirow{8}{*}{ Broth microdilution } & & \multirow{8}{*}{ [96] } \\
\hline & & B. cereus ATCC 11778 & 9.77 & & & & \\
\hline & & S. aureus ATCC 6538 & 8.14 & & & & \\
\hline & & E. faecalis ATCC 19433 & 11.39 & & & & \\
\hline & & L. sakei ATCC 15521 & 8.14 & & & & \\
\hline & & L. lactis ATCC 19435 & 8.14 & & & & \\
\hline & & S. typhimurium ATCC 14028 & 625 & & & & \\
\hline & & C. freundii ATCC 27853 & 5000 & & & & \\
\hline \multirow{5}{*}{ Gentamycin } & & S. typhimurium ATCC 14028 & 0.25 & & \multirow{5}{*}{ Broth microdilution } & & \multirow{5}{*}{ [81] } \\
\hline & & E. coli ATCC 25922 & 0.25 & & & & \\
\hline & & C. freundii ATCC 27853 & 1 & & & & \\
\hline & & B. subtilis КCТC1914 & 1 & & & & \\
\hline & & S. typhimurium KCTC1926 & 0.5 & & & & \\
\hline \multirow{3}{*}{ Kanamycin } & & S. aureus 209 KCTC1916 & 2 & & \multirow{3}{*}{ Broth microdilution } & & \multirow{3}{*}{ [81] } \\
\hline & & B. subtilis КСТC1914 & 2 & & & & \\
\hline & & S. typhimurium КСТС1926 & 1 & & & & \\
\hline \multirow{6}{*}{ Fuconazole } & & C. tropicalis 17A & 0.125 (MBEC 4) & & \multirow{6}{*}{ Broth microdilution } & \multirow{6}{*}{$\begin{array}{l}\text { The clinical isolates } 72 \mathrm{~A}, 72 \mathrm{P}, \\
\text { and } 94 \mathrm{P} \text { are resistant to } \\
\text { fluconazole, amphotericin } \mathrm{B}, \\
\text { voriconazole and anidulafungin. }\end{array}$} & \multirow{6}{*}{ [88] } \\
\hline & & C. tropicalis 57A & 0.125 (MBEC 64) & & & & \\
\hline & & C. tropicalis $72 \mathrm{~A}$ & 128 (MBEC 8) & & & & \\
\hline & & C. tropicalis $72 \mathrm{P}$ & 128 (MBEC 128) & & & & \\
\hline & & C. tropicalis $94 \mathrm{P}$ & 64 (MBEC 32) & & & & \\
\hline & & C. tropicalis $102 \mathrm{~A}$ & 0.125 (MBEC 128) & & & & \\
\hline \multirow{4}{*}{ Colistin } & & E. coli ATCC 25922 & 2 & & \multirow{4}{*}{ Broth microdilution } & & \multirow{4}{*}{ [91] } \\
\hline & & P. aeruginosa ATCC 27853 & 1 & & & & \\
\hline & & K. pneumonia ATCC BAA-1705 & 2 & & & & \\
\hline & & A. baumannii $\mathrm{AB} 01$ & 4 & & & & \\
\hline
\end{tabular}


Table 2. Cont

\begin{tabular}{|c|c|c|c|c|c|c|c|}
\hline IL & Acronym & Species & $\mathrm{MIC}, \mu \mathrm{g} \mathrm{mL}^{-1}$ & $\mathrm{MBC}, \mu \mathrm{g} \mathrm{mL}^{-1}$ & Method & Notes & Ref. \\
\hline \multirow{6}{*}{ Vancomycin } & & B. subtilis ATCC 6633 & $<0.48$ & & \multirow{6}{*}{ Broth microdilution } & & \multirow{6}{*}{ [92] } \\
\hline & & K. pneumonia АТСС 9997 & 15.62 & & & & \\
\hline & & E. faecalis ATCC 29212 & 1.95 & & & & \\
\hline & & VRE ATCC 51299 & 3.91 & & & & \\
\hline & & S. aureus & 7.82 & & & & \\
\hline & & MRSA CIP 106760 & 3.91 & & & & \\
\hline \multirow{2}{*}{\multicolumn{2}{|c|}{ Rifampicin }} & M. smegmatis ATCC 607 & $<0.48$ & & \multirow{2}{*}{ Broth microdilution } & & \multirow{2}{*}{ [92] } \\
\hline & & E. coli ATCC 25922 & 0.98 & & & & \\
\hline Norfloxacin & & P. aeruginosa ATCC 27853 & $<0.48$ & & Broth microdilution & & [92] \\
\hline \multirow{2}{*}{ Amphotericin B } & & C. albicans ATCC 10231 & $<0.48$ & & \multirow[b]{2}{*}{ Broth microdilution } & & \multirow[b]{2}{*}[92]{} \\
\hline & & S. cerevisiae ATCC 2601 & $<0.48$ & & & & \\
\hline
\end{tabular}

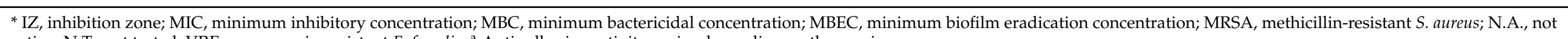
active; N.T., not tested; VRE, vancomycin-resistant E. faecalis. ${ }^{\text {a }}$ Anti-adhesive activity varies depending on the species. 
Table 3. Antimicrobial activity of API-ILs *.

\begin{tabular}{|c|c|c|c|c|c|c|c|c|}
\hline IL & Acronym & Species & $\mathrm{IZ}, \mathrm{mm}$ & $\mathrm{MIC} \mu \mathrm{g} \mathrm{mL}^{-1}$ & $\mathrm{MBC}_{,} \mu \mathrm{g} \mathrm{mL}^{-1}$ & Method & Notes & Ref. \\
\hline \multirow{4}{*}{$\begin{array}{c}\text { 1-Ethyl-3- } \\
\text { methylimidazolium } \\
\text { nalidixate }\end{array}$} & \multirow{4}{*}[\mathrm{C}_{2}\mathrm{Mim}]{$[\mathrm{Nal}]$} & E. coli BW25113 (wild-type) & 11 & & & \multirow{4}{*}{$\begin{array}{l}\text { Disk diffusion test, } \\
10 \mu \mathrm{g} \text { per disk }\end{array}$} & \multirow{4}{*}{$\begin{array}{l}\text { Deletions } \Delta \mathrm{rfaC}, \Delta \mathrm{rfaL}, \\
\text { and } \Delta \mathrm{rfaG} \text { affect the cell } \\
\text { surface hydrophobicity } \\
\text { and membrane } \\
\text { permeability. }\end{array}$} & \multirow{4}{*}{ [86] } \\
\hline & & E. coli JW3596 $(\Delta \mathrm{rfaC})$ & 20 & & & & & \\
\hline & & E. coli JW3597 $(\Delta \mathrm{rfaL})$ & 11 & & & & & \\
\hline & & E. coli JW3606 ( $\Delta \mathrm{rfaG})$ & 18 & & & & & \\
\hline \multirow{4}{*}{$\begin{array}{l}\text { 1-Hexadecyl-3- } \\
\text { methylimidazolium } \\
\text { ampicillinate }\end{array}$} & \multirow{4}{*}[\mathrm{C}_{16}\mathrm{Mim}]{$[\mathrm{Amp}]$} & S. aureus ATCC 6538 & & $30 \mu \mathrm{M}$ & & \multirow{4}{*}{ Broth microdilution } & & \multirow{4}{*}{ [97] } \\
\hline & & E. coli O157:H7 ATCC 43895 & & $9 \mu \mathrm{M}$ & & & & \\
\hline & & E. faecium ATCC 49474 & & $13 \mu \mathrm{M}$ & & & & \\
\hline & & K. pneumonia ATCC 4352 & & $15 \mu \mathrm{M}$ & & & & \\
\hline \multirow{4}{*}{$\begin{array}{l}\text { 1-Hexadecyl-2,3- } \\
\text { dimethylimidazolium } \\
\text { ampicillinate }\end{array}$} & \multirow{4}{*}{ [C $\left.{ }_{16} \mathrm{MMim}\right][\mathrm{Amp}]$} & S. aureus ATCC 6538 & & $14 \mu \mathrm{M}$ & & \multirow{4}{*}{ Broth microdilution } & & \multirow{4}{*}{ [97] } \\
\hline & & E. coli O157:H7 ATCC 43895 & & $9 \mu \mathrm{M}$ & & & & \\
\hline & & E. faecium ATCC 49474 & & $0.4 \mu \mathrm{M}$ & & & & \\
\hline & & K. pneumonia ATCC 4352 & & $15 \mu \mathrm{M}$ & & & & \\
\hline \multirow{11}{*}{$\begin{array}{l}\text { 1-Hexadecylpyridi- } \\
\text { nium ampicillinate }\end{array}$} & \multirow{11}{*}[\mathrm{C}_{16}\mathrm{Py}]{$[\mathrm{Amp}]$} & S. aureus ATCC 6538 & & $8 \mu \mathrm{M}$ & & \multirow{11}{*}{ Broth microdilution } & \multirow{11}{*}{$\begin{array}{l}\text { E. coli TEM CTX M9, CTX } \\
\text { M2, and AmpC MOX2 } \\
\text { are ampicillin-resistant } \\
\text { strains. }\end{array}$} & \multirow{11}{*}[82,97]{} \\
\hline & & S. aureus ATCC 25293 & & $5 \mu \mathrm{M}$ & & & & \\
\hline & & S. epidermidis (clinical isolate) & & $5 \mu \mathrm{M}$ & & & & \\
\hline & & E. coli ATCC 25922 & & $500 \mu \mathrm{M}$ & & & & \\
\hline & & E. coli TEM CTX M9 & & $5 \mu \mathrm{M}$ & & & & \\
\hline & & E. coli CTX M2 & & $50 \mu \mathrm{M}$ & & & & \\
\hline & & E. coli AmpC MOX2 & & $>5000 \mu \mathrm{M}$ & & & & \\
\hline & & E. faecium ATCC 49474 & & $0.4 \mu \mathrm{M}$ & & & & \\
\hline & & E. faecalis (clinical isolate) & & $5 \mu \mathrm{M}$ & & & & \\
\hline & & K. pneumonia ATCC 4352 & & $9 \mu \mathrm{M}$ & & & & \\
\hline & & K. pneumoniae (clinical isolate) & & $50 \mu \mathrm{M}$ & & & & \\
\hline \multirow{4}{*}{$\begin{array}{l}N \text {-Ethyl- } N- \\
\text { methylpiperidinium } \\
\text { nalidixate }\end{array}$} & \multirow{4}{*}[\mathrm{C}_{2}\mathrm{C}_{1}\mathrm{Pip}]{$[\mathrm{Nal}]$} & E. coli BW25113 (wild-type) & 12.9 & & & \multirow{4}{*}{$\begin{array}{l}\text { Disk diffusion test, } \\
10 \mu \mathrm{g} \text { per disk }\end{array}$} & \multirow{4}{*}{$\begin{array}{l}\text { Deletions } \Delta \mathrm{rfaC}, \Delta \mathrm{rfaL}, \\
\text { and } \Delta \mathrm{rfaG} \text { affect the cell } \\
\text { surface hydrophobicity } \\
\text { and membrane } \\
\text { permeability. }\end{array}$} & \multirow{4}{*}{ [86] } \\
\hline & & E. coli JW3596 $(\Delta \mathrm{rfaC})$ & 22.9 & & & & & \\
\hline & & E. coli JW3597 ( $(\mathrm{rfaL})$ & 12.8 & & & & & \\
\hline & & E. coli JW3606 ( $(\mathrm{rfaG})$ & 21 & & & & & \\
\hline
\end{tabular}


Table 3. Cont.

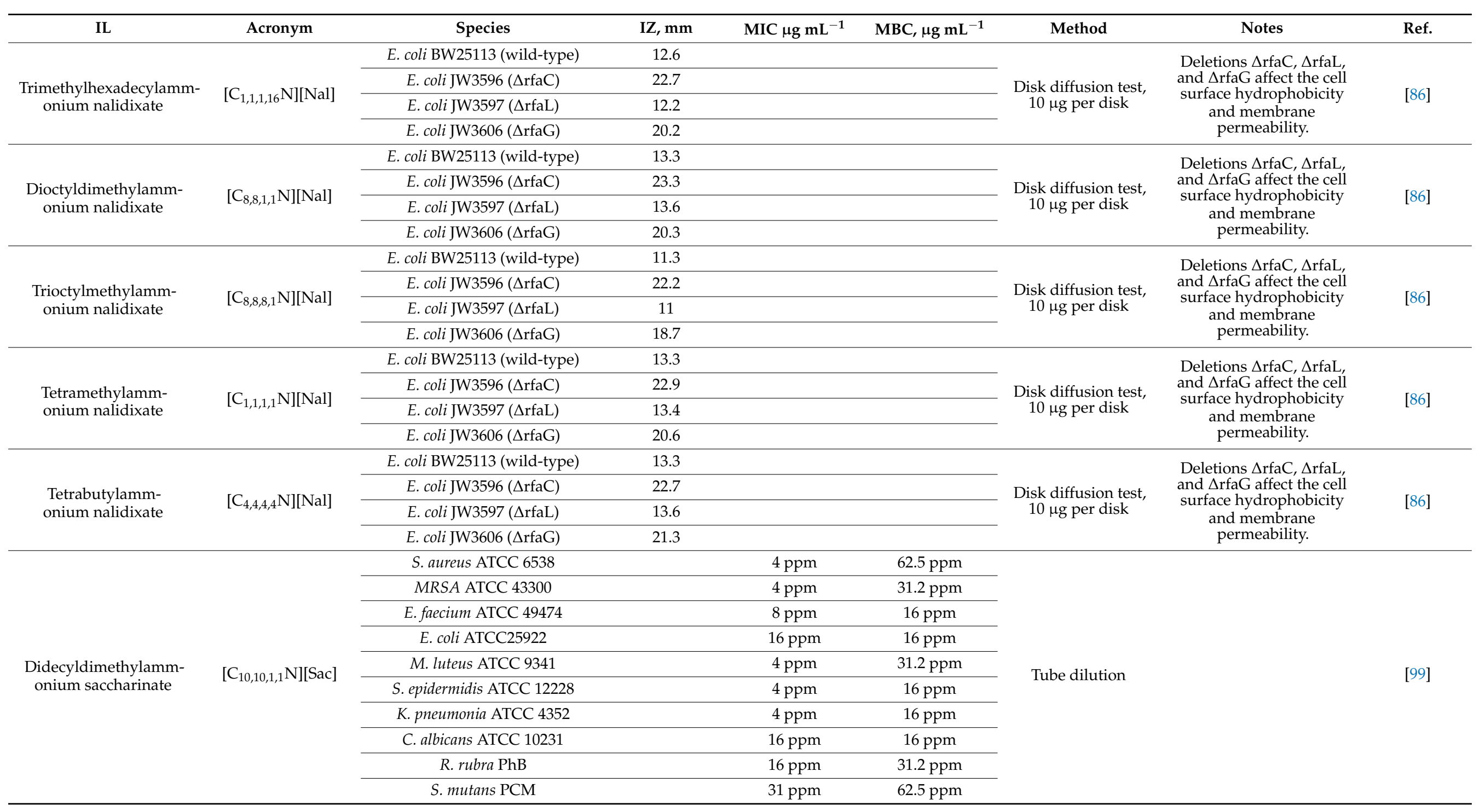


Table 3. Cont.

\begin{tabular}{|c|c|c|c|c|c|c|c|c|}
\hline IL & Acronym & Species & $\mathrm{IZ}, \mathrm{mm}$ & MIC $\mu \mathrm{g} \mathrm{mL}^{-1}$ & $\mathrm{MBC}, \mu \mathrm{g} \mathrm{mL} \mathrm{L}^{-1}$ & Method & Notes & Ref. \\
\hline \multirow{9}{*}{$\begin{array}{l}\text { Didecyldimethylamm- } \\
\text { onium acesulfamate }\end{array}$} & \multirow{9}{*}[\mathrm{C}_{10,10,1,1}\mathrm{N}]{$[$ Ace $]$} & S. aureus ATCC 6538 & & $8 \mathrm{ppm}$ & $16 \mathrm{ppm}$ & \multirow{9}{*}{ Tube dilution } & & \multirow{9}{*}{ [99] } \\
\hline & & MRSA ATCC 43300 & & $4 \mathrm{ppm}$ & $31.2 \mathrm{ppm}$ & & & \\
\hline & & E. faecium ATCC 49474 & & $8 \mathrm{ppm}$ & $31.2 \mathrm{ppm}$ & & & \\
\hline & & E. coli ATCC25922 & & $16 \mathrm{ppm}$ & $62.5 \mathrm{ppm}$ & & & \\
\hline & & M. luteus ATCC 9341 & & $8 \mathrm{ppm}$ & $62.5 \mathrm{ppm}$ & & & \\
\hline & & S. epidermidis ATCC 12228 & & $4 \mathrm{ppm}$ & $31.2 \mathrm{ppm}$ & & & \\
\hline & & K. pneumonia ATCC 4352 & & $4 \mathrm{ppm}$ & $31.2 \mathrm{ppm}$ & & & \\
\hline & & R. rubra $\mathrm{PhB}$ & & $16 \mathrm{ppm}$ & $62.5 \mathrm{ppm}$ & & & \\
\hline & & S. mutans PCM & & $16 \mathrm{ppm}$ & $125 \mathrm{ppm}$ & & & \\
\hline \multirow{4}{*}{$\begin{array}{l}\text { Tetrabutylphosphonium } \\
\text { nalidixate }\end{array}$} & \multirow{4}{*}[\mathrm{C}_{4,4,4,4}\mathrm{P}]{$[\mathrm{Nal}]$} & E. coli BW25113 (wild-type) & 13.3 & & & \multirow{4}{*}{$\begin{array}{l}\text { Disk diffusion test, } \\
10 \mu \mathrm{g} \text { per disk }\end{array}$} & \multirow{4}{*}{$\begin{array}{l}\text { Deletions } \Delta \mathrm{rfaC}, \Delta \mathrm{rfaL}, \\
\text { and } \Delta \mathrm{rfaG} \text { affect the cell } \\
\text { surface hydrophobicity } \\
\text { and membrane } \\
\text { permeability. }\end{array}$} & \multirow{4}{*}{ [86] } \\
\hline & & E. coli JW3596 $(\Delta \mathrm{rfaC})$ & 22.6 & & & & & \\
\hline & & E. coli JW3597 ( $\Delta \mathrm{rfaL})$ & 12.9 & & & & & \\
\hline & & E. coli JW3606 $(\Delta \mathrm{rfaG})$ & 20.4 & & & & & \\
\hline \multirow{7}{*}{$\begin{array}{l}\text { Trihexyltetradecylphos- } \\
\text { phonium } \\
\text { ampicillinate }\end{array}$} & \multirow{7}{*}[\mathrm{C}_{6,6,6,6,14}\mathrm{P}]{$[\mathrm{Amp}]$} & E. coli ATCC 25922 & & $2500 \mu \mathrm{M}$ & & \multirow{7}{*}{ Broth microdilution } & \multirow{7}{*}{$\begin{array}{l}\text { E. coli TEM CTX M9, CTX } \\
\text { M2, and AmpC MOX2 are } \\
\text { ampicillin-resistant strains. }\end{array}$} & \multirow{7}{*}{ [82] } \\
\hline & & E. coli TEM CTX M9 & & $500 \mu \mathrm{M}$ & & & & \\
\hline & & E. coli AmpC MOX2 & & $>5000 \mu \mathrm{M}$ & & & & \\
\hline & & K. pneumoniae (clinical isolate) & & $5000 \mu \mathrm{M}$ & & & & \\
\hline & & S. aureus ATCC 25293 & & $50 \mu \mathrm{M}$ & & & & \\
\hline & & S. epidermidis (clinical isolate) & & $50 \mu \mathrm{M}$ & & & & \\
\hline & & E. faecalis (clinical isolate) & & $50 \mu \mathrm{M}$ & & & & \\
\hline \multirow{10}{*}{$\begin{array}{l}\text { Benzalkonium } \\
\text { saccharinate }\end{array}$} & \multirow{10}{*}[\mathrm{BA}]{$[\mathrm{Sac}]$} & S. aureus ATCC 6538 & & $4 \mathrm{ppm}$ & $31.2 \mathrm{ppm}$ & \multirow{10}{*}{ Tube dilution } & & \multirow{10}{*}{ [99] } \\
\hline & & MRSA ATCC 43300 & & $4 \mathrm{ppm}$ & $31.2 \mathrm{ppm}$ & & & \\
\hline & & E. faecium ATCC 49474 & & $8 \mathrm{ppm}$ & $16 \mathrm{ppm}$ & & & \\
\hline & & E. coli ATCC25922 & & $16 \mathrm{ppm}$ & $62.5 \mathrm{ppm}$ & & & \\
\hline & & M. luteus ATCC 9341 & & $8 \mathrm{ppm}$ & $62.5 \mathrm{ppm}$ & & & \\
\hline & & S. epidermidis ATCC 12228 & & $4 \mathrm{ppm}$ & $31.2 \mathrm{ppm}$ & & & \\
\hline & & K. pneumonia ATCC 4352 & & $4 \mathrm{ppm}$ & $62.5 \mathrm{ppm}$ & & & \\
\hline & & C. albicans ATCC 10231 & & $16 \mathrm{ppm}$ & $31.2 \mathrm{ppm}$ & & & \\
\hline & & R. rubra $\mathrm{PhB}$ & & $16 \mathrm{ppm}$ & $62.5 \mathrm{ppm}$ & & & \\
\hline & & S. mutans PCM & & $0.1 \mathrm{ppm}$ & $0.5 \mathrm{ppm}$ & & & \\
\hline
\end{tabular}


Table 3. Cont.

\begin{tabular}{|c|c|c|c|c|c|c|c|c|}
\hline IL & Acronym & Species & $\mathrm{IZ}, \mathrm{mm}$ & MIC $\mu \mathrm{g} \mathrm{mL}^{-1}$ & $\mathrm{MBC}, \mu \mathrm{g} \mathrm{mL} \mathrm{L}^{-1}$ & Method & Notes & Ref. \\
\hline \multirow{10}{*}{$\begin{array}{l}\text { Benzalkonium } \\
\text { acesulfamate }\end{array}$} & \multirow{10}{*}{ [BA][Ace] } & S. aureus ATCC 6538 & & $4 \mathrm{ppm}$ & $31.2 \mathrm{ppm}$ & \multirow{10}{*}{ Tube dilution } & & \multirow{10}{*}{ [99] } \\
\hline & & MRSA ATCC 43300 & & $4 \mathrm{ppm}$ & $31.2 \mathrm{ppm}$ & & & \\
\hline & & E. faecium ATCC 49474 & & $8 \mathrm{ppm}$ & $31.2 \mathrm{ppm}$ & & & \\
\hline & & E. coli ATCC25922 & & $31 \mathrm{ppm}$ & $125 \mathrm{ppm}$ & & & \\
\hline & & M. luteus ATCC 9341 & & $8 \mathrm{ppm}$ & $62.5 \mathrm{ppm}$ & & & \\
\hline & & S. epidermidis ATCC 12228 & & $4 \mathrm{ppm}$ & $62.5 \mathrm{ppm}$ & & & \\
\hline & & K. pneumonia АТCC 4352 & & $8 \mathrm{ppm}$ & $31.2 \mathrm{ppm}$ & & & \\
\hline & & C. albicans ATCC 10231 & & $16 \mathrm{ppm}$ & $31.2 \mathrm{ppm}$ & & & \\
\hline & & R. rubra $\mathrm{PhB}$ & & $16 \mathrm{ppm}$ & $62.5 \mathrm{ppm}$ & & & \\
\hline & & S. mutans PCM & & $1 \mathrm{ppm}$ & $16 \mathrm{ppm}$ & & & \\
\hline \multirow{4}{*}{ Nalidixic acid } & & E. coli BW25113 (wild-type) & 11 & & & \multirow{4}{*}{$\begin{array}{l}\text { Disk diffusion test, } \\
10 \mu \mathrm{g} \text { per disk }\end{array}$} & \multirow{4}{*}{$\begin{array}{l}\text { Deletions } \Delta \mathrm{rfaC}, \Delta \mathrm{rfaL}, \\
\text { and } \Delta \mathrm{rfaG} \text { affect the cell } \\
\text { surface hydrophobicity } \\
\text { and membrane } \\
\text { permeability. }\end{array}$} & \multirow{4}{*}{ [86] } \\
\hline & & E. coli JW3596 $(\Delta \mathrm{rfaC})$ & 20 & & & & & \\
\hline & & E. coli JW3597 ( $\Delta \mathrm{rfaL})$ & 11 & & & & & \\
\hline & & E. coli JW3606 $(\Delta \mathrm{rfaG})$ & 18 & & & & & \\
\hline \multirow{11}{*}{$\begin{array}{l}\text { Ampicillin sodium } \\
\text { salt }\end{array}$} & & S. aureus ATCC 6538 & & $27 \mu \mathrm{M}$ & & \multirow{11}{*}{ Broth microdilution } & \multirow{11}{*}{$\begin{array}{l}\text { E. coli TEM CTX M9, CTX } \\
\text { M2, and AmpC MOX2 } \\
\text { are ampicillin-resistant } \\
\text { strains. }\end{array}$} & \multirow{11}{*}[82,97]{} \\
\hline & & S. epidermidis (clinical isolate) & & $50 \mu \mathrm{M}$ & & & & \\
\hline & & E. coli O157:H7 ATCC 43895 & & $12 \mu \mathrm{M}$ & & & & \\
\hline & & E. coli ATCC 25922 & & $50 \mu \mathrm{M}$ & & & & \\
\hline & & E. coli TEM CTX M9 & & $>5000 \mu \mathrm{M}$ & & & & \\
\hline & & E. coli CTX M2 & & $>5000 \mu \mathrm{M}$ & & & & \\
\hline & & E. coli AmpC MOX2 & & $>5000 \mu \mathrm{M}$ & & & & \\
\hline & & E. faecium ATCC 49474 & & $17 \mu \mathrm{M}$ & & & & \\
\hline & & E. faecalis (clinical isolate) & & $50 \mu \mathrm{M}$ & & & & \\
\hline & & K. pneumonia ATCC 4352 & & $20 \mu \mathrm{M}$ & & & & \\
\hline & & K. pneumoniae (clinical isolate) & & $2500 \mu \mathrm{M}$ & & & & \\
\hline
\end{tabular}


Table 3. Cont.

\begin{tabular}{|c|c|c|c|c|c|c|c|c|}
\hline IL & Acronym & Species & $\mathrm{IZ}, \mathrm{mm}$ & MIC $\mu \mathrm{g} \mathrm{mL}^{-1}$ & $\mathrm{MBC}, \mu \mathrm{g} \mathrm{mL}^{-1}$ & Method & Notes & Ref. \\
\hline \multirow{13}{*}{$\begin{array}{l}\text { Benzalkonium } \\
\text { chloride }\end{array}$} & & S. aureus ATCC 6538 & & $2 \mathrm{ppm}$ & $62.5 \mathrm{ppm}$ & \multirow{13}{*}{$\begin{array}{l}\text { Tube dilution, broth } \\
\text { microdilution }\end{array}$} & & \multirow{13}{*}[81,99]{} \\
\hline & & MRSA ATCC 43300 & & $2 \mathrm{ppm}$ & $31.2 \mathrm{ppm}$ & & & \\
\hline & & S. aureus 209 КСТC1916 & & 8 & & & & \\
\hline & & S. aureus R209 KCTC1928 & & 8 & & & & \\
\hline & & E. faecium ATCC 49474 & & $4 \mathrm{ppm}$ & $31.2 \mathrm{ppm}$ & & & \\
\hline & & E. coli ATCC25922 & & $8 \mathrm{ppm}$ & $62.5 \mathrm{ppm}$ & & & \\
\hline & & M. luteus ATCC 9341 & & $4 \mathrm{ppm}$ & $31.2 \mathrm{ppm}$ & & & \\
\hline & & S. epidermidis ATCC 12228 & & $2 \mathrm{ppm}$ & $16 \mathrm{ppm}$ & & & \\
\hline & & K. pneumonia ATCC 4352 & & $4 \mathrm{ppm}$ & $31.2 \mathrm{ppm}$ & & & \\
\hline & & B. subtilis KCTC1914 & & 8 & & & & \\
\hline & & C. albicans ATCC 10231 & & $8 \mathrm{ppm}$ & $16 \mathrm{ppm}$ & & & \\
\hline & & R. rubra $\mathrm{PhB}$ & & $8 \mathrm{ppm}$ & $31.2 \mathrm{ppm}$ & & & \\
\hline & & S. mutans PCM & & $2 \mathrm{ppm}$ & 16 ppm & & & \\
\hline \multirow{9}{*}{$\begin{array}{l}\text { Didecyldimethylamm- } \\
\text { onium chloride }\end{array}$} & & S. aureus ATCC 6538 & & $2 \mathrm{ppm}$ & $31.2 \mathrm{ppm}$ & \multirow{9}{*}{ Tube dilution } & & \multirow{9}{*}{ [99] } \\
\hline & & MRSA ATCC 43300 & & $2 \mathrm{ppm}$ & $31.2 \mathrm{ppm}$ & & & \\
\hline & & E. coli ATCC25922 & & $8 \mathrm{ppm}$ & $31.2 \mathrm{ppm}$ & & & \\
\hline & & M. luteus ATCC 9341 & & $2 \mathrm{ppm}$ & $31.2 \mathrm{ppm}$ & & & \\
\hline & & S. epidermidis ATCC 12228 & & $2 \mathrm{ppm}$ & $31.2 \mathrm{ppm}$ & & & \\
\hline & & K. pneumonia ATCC 4352 & & $4 \mathrm{ppm}$ & $16 \mathrm{ppm}$ & & & \\
\hline & & C. albicans ATCC 10231 & & $8 \mathrm{ppm}$ & 16 ppm & & & \\
\hline & & R. rubra $\mathrm{PhB}$ & & $4 \mathrm{ppm}$ & $31.2 \mathrm{ppm}$ & & & \\
\hline & & S. mutans PCM & & $2 \mathrm{ppm}$ & $16 \mathrm{ppm}$ & & & \\
\hline
\end{tabular}




\subsubsection{Double-Charged QACs (Bis-QACs)}

Bis-QAC (or so-called "twin surfactants") is a subclass of synthetic amphiphiles that contain two cationic nitrogen atoms, a spacer linking them, and two lipophilic alkyl substituents [100]. These are common characteristics of typical bis-QAC, the exact structure of which can vary greatly. The intense development of bis-QACs began later than that of mono-QACs in the 1980s with the discovery of octenidine (see the Commercial QACs section). Nonetheless, there are many publications on the synthesis and biocide properties of bis-QACs.

A significant number of alkyl bis-QACs were synthesized to test the effect of the total charge of the molecule on the activity (Figure 20).

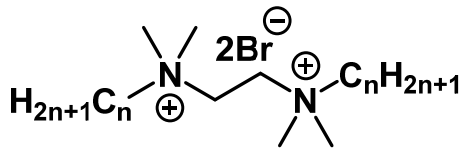

(42)<smiles>CCCCCCCCC[N+](C)(C)CC[N+](C)(C)C</smiles>

(43)

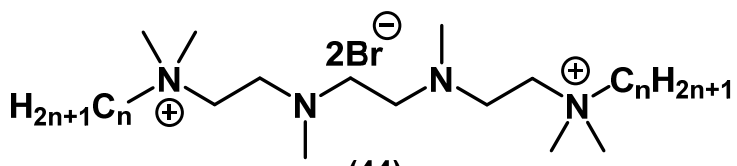

(44)

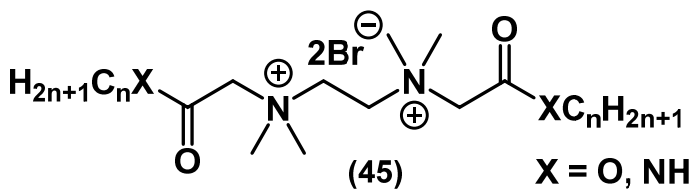<smiles>CCCN(C)CCOCC(=O)OCCN(C)C</smiles>

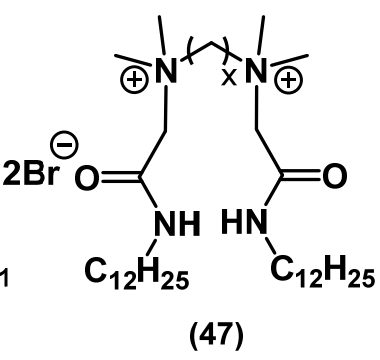

Figure 20. Alkyl bis-QACs.

Bis-QACs with ester spacer $\mathbf{4 6}$ showed better activity than their mono analogues, both against Gram-positive and Gram-negative bacteria and fungi [101]. It is worth noting that the activity against $E$. coli was nonlinear and plummeted upon increasing the alkyl chain length from $C_{12}$ to $C_{14}$. This relationship, which is known for the biocidal action of amphiphils on Gram-negative bacteria, is called the "cut-off" effect. It was described by Devinsky and colleagues as a consequence of membrane penetration [102]. The addition of a second charged nitrogen atom increased the activity 3-fold in S. aureus and 4-fold in E. coli in the work of Hodye (substance 47). The activity also correlated with the distance between the heads, with the optimal spacer length being $C_{6}$ [103]. Wuest and Minbiole and colleagues studied the biocidal action of QACs based on polyamines 43-44 [71,104]. Tetramethylethylenediamine derivatives (TMEDAs) 42 turned out to be an extremely promising class of biocides because of their simple synthesis, cheap starting materials, and high activity [75]. In all the above-mentioned studies, the biological effect on pathogenic bacteria increased 3-4 times, especially for Gram-negative strains, compared to mono-QACs.

Changing the spacer in the bis-QAC structure is one of the key factors in the design of target molecules. Thus, the aforementioned alkyl bis-QACs can contain aromatic spacers (Figure 21). 


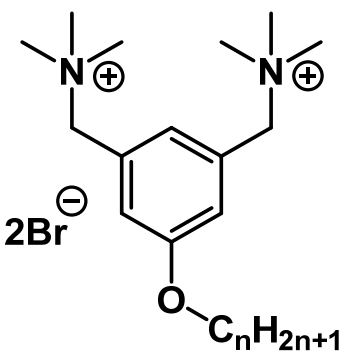

$\mathrm{n}=14,16$

(48)

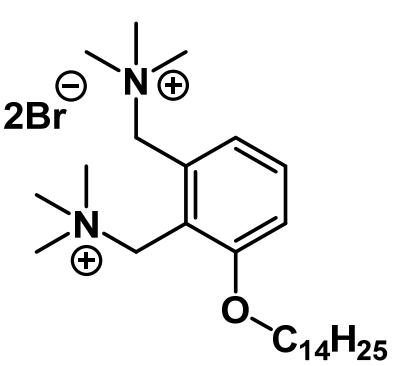

(51)

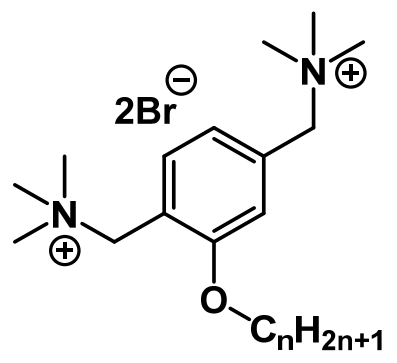

$n=10,12,14,16,18$

(49)<smiles>CCCCCCCCCCCCCCCCCCCN(C)C</smiles>

(52)<smiles>CCCCCCCCCc1cccc(C[N+](C)(C)C)c1OCCCC</smiles>

(50)<smiles>[R]C1([R2])OCc2c(C([O-])Cl)cnc(C[N+]([R3])([R3])C)c2O1</smiles>

(54)

$\mathrm{R}_{1}=\mathrm{H}, \mathrm{CH}_{3}$;

$\mathrm{R}_{2}=\mathrm{H}, \mathrm{CH}_{3}, \mathrm{C}_{2} \mathrm{H}_{5}, \mathrm{C}_{3} \mathrm{H}_{7}, \mathrm{CH}\left(\mathrm{CH}_{3}\right)_{2}$,

$\mathrm{C}_{4} \mathrm{H}_{9}, \mathrm{C}\left(\mathrm{CH}_{3}\right)_{3}, \mathrm{CH}\left(\mathrm{CH}_{3}\right) \mathrm{C}_{2} \mathrm{H}_{5}, \mathrm{C}_{5} \mathrm{H}_{11}$, $\mathrm{CH}\left(\mathrm{CH}_{3}\right) \mathrm{C}_{3} \mathrm{H}_{7}, \mathrm{CH}\left(\mathrm{C}_{2} \mathrm{H}_{5}\right) \mathrm{C}_{2} \mathrm{H}_{5}$, $\mathrm{C}_{6} \mathrm{H}_{13}, \mathrm{C}_{7} \mathrm{H}_{15}, \mathrm{CH}\left(\mathrm{C}_{2} \mathrm{H}_{5}\right) \mathrm{C}_{4} \mathrm{H}_{9}, \mathrm{C}_{8} \mathrm{H}_{17}$ $\mathrm{CH}\left(\mathrm{CH}_{3}\right) \mathrm{C}_{9} \mathrm{H}_{19}$;

$\mathrm{R}_{3}=\mathrm{C}_{8} \mathrm{H}_{17}, \mathrm{C}_{10} \mathrm{H}_{21}, \mathrm{C}_{12} \mathrm{H}_{25}, \mathrm{C}_{14} \mathrm{H}_{29}$, $\mathrm{C}_{16} \mathrm{H}_{33}, \mathrm{C}_{18} \mathrm{H}_{37}$.

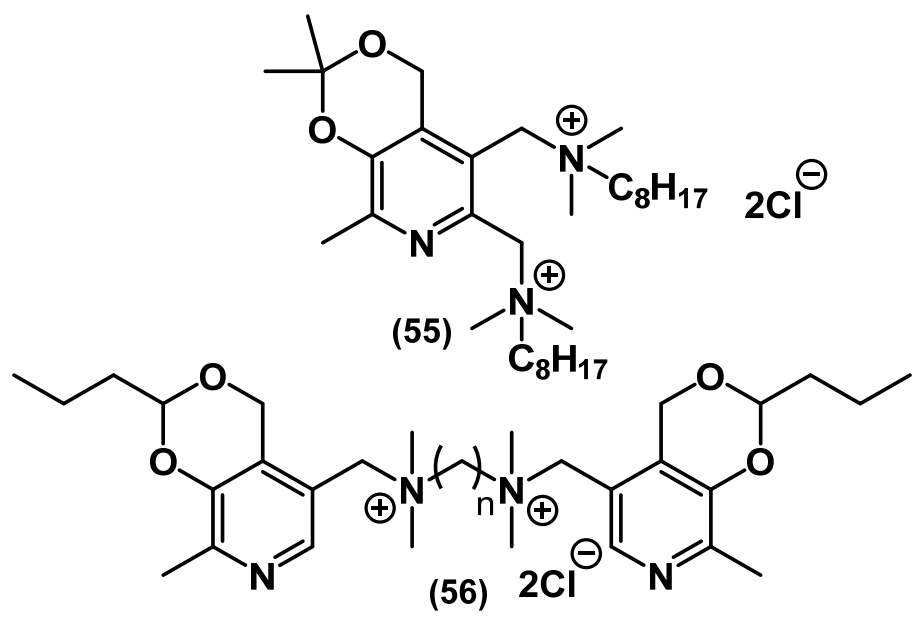

Figure 21. Alkyl bis-QACs containing aromatic spacers.

A study by LaDow and colleagues showed that bis-QACs 48-52 inhibited the growth of Gram-positive bacteria at approximately the same concentration as their mono analogs. However, bis-QACs had a much stronger effect on Gram-negative bacteria, which was confirmed by other studies [105]. In continuation of their work on the study of pyridoxine QAC derivatives, Shtyrlun and colleagues noted a clear dependence of the activity of compounds $\mathbf{5 4}$ on their lipophilicity. Thus, the values of the lipophilicity coefficient for the most active compounds $\left(\mathrm{C}_{10}, \mathrm{C}_{12}\right)$ were in the range of 1 to 3 ; at values higher than 6 or lower than 0 , the activity decreased sharply [106]. Forman and colleagues studied QAC derivatives of malachite green 53, comparing its mono- and bis-QACs. Analogs with two long alkyl chains were generally comparable to mono-QACs but were more efficient against resistant bacteria [107].

Similar to mono-QACs, the head of bis-QACs can have a saturated heterocyclic structure (Figure 22). 


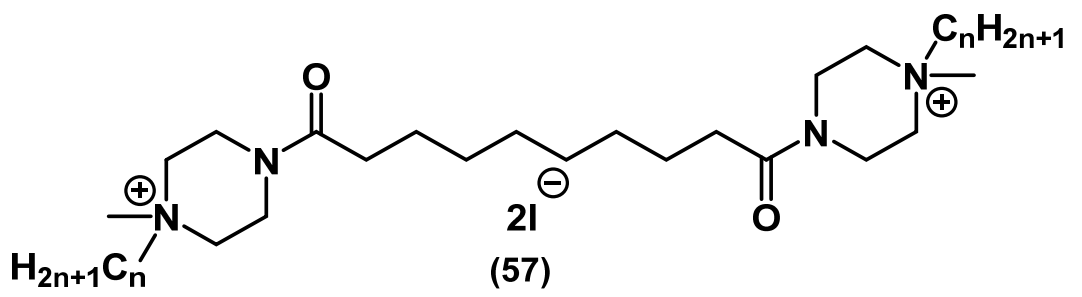

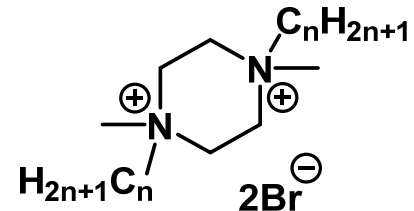

(58)

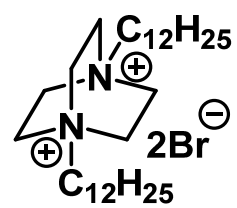

(59)

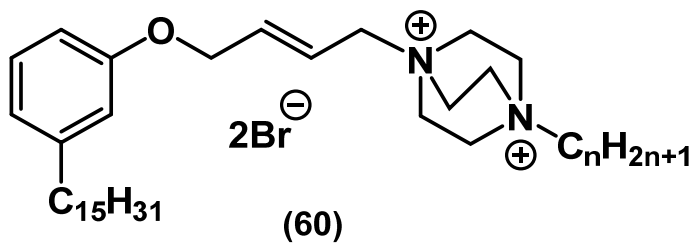

Figure 22. Bis-QACs containing saturated heterocycles.

Kourai and colleagues, in their study of bis-QAC derivatives of piperazine 57, found that compounds with different spacer structures but the same lipophilicity exhibited different activities. This fact suggested that the dependence of the biocidal action on lipophilicity was valid only for the series of QACs differing in the length of the tail [108]. Kontos and colleagues tested the dependence of the activity of 58-59 on the rigidity of the structure. The initial assumption that a more flexible structure would provide easier passage through the bacterial membrane and accelerate cell lysis turned out to be erroneous. Thus, derivatives of the more rigid amine structure $\mathbf{5 9}$ of diazobicyclooctane (DABCO) were most active in the series [109]. A series of heterocyclic QACs based on cardanol 60 was developed by Ma and colleagues [110]. Along with moderate antibacterial activity, the compounds appeared to be good surfactants.

There are several examples of mixed bis-QACs carrying two different heterocycles or heterocyclic and alkyl parts (Figure 23).

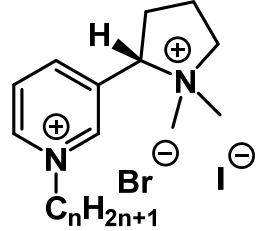

(61)<smiles></smiles>

(62)<smiles></smiles>

(63)

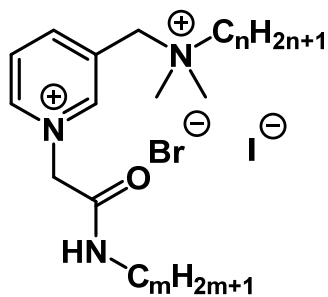

(64)<smiles>CCC[N+]1(C)CCN(CC[N+](C)(C)CC)CC1</smiles>

(65)

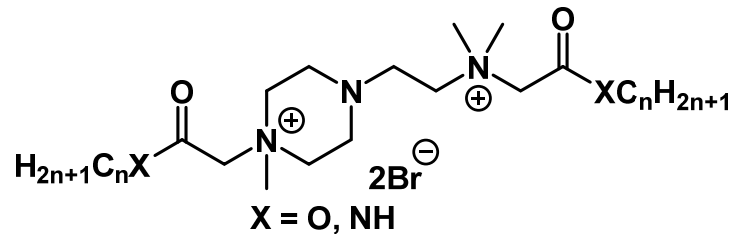

(66)

Figure 23. Mixed bis-QACs. 
In the continuation of the work on preparation of the above-mentioned QAC derivatives of quinine and nicotine, the usual "activation" of the second nitrogen charged center did not lead to a significant increase in the activity of 61-62. Presumably, the total charge of the molecule does not affect the activity as strongly as the addition of the second alkyl chain [74]. In the work of Schallenhammer and colleagues, hybrid bis-QACs 63-64 combining CPC 5 and BAC 1 showed higher activity against Gram-negative bacteria than each of the commercial "source drugs" applied separately. At the same time, hybrid monoderivatives did not show such a result [111]. Piperazine bis-QAC derivatives 65 and their "soft" analogs 66 showed similar relationships with the previous bis-QACs [72,112].

Additionally, there is a range of interesting works concerning QACs with polynuclear heterocycles with several heteroatoms (Figure 24).

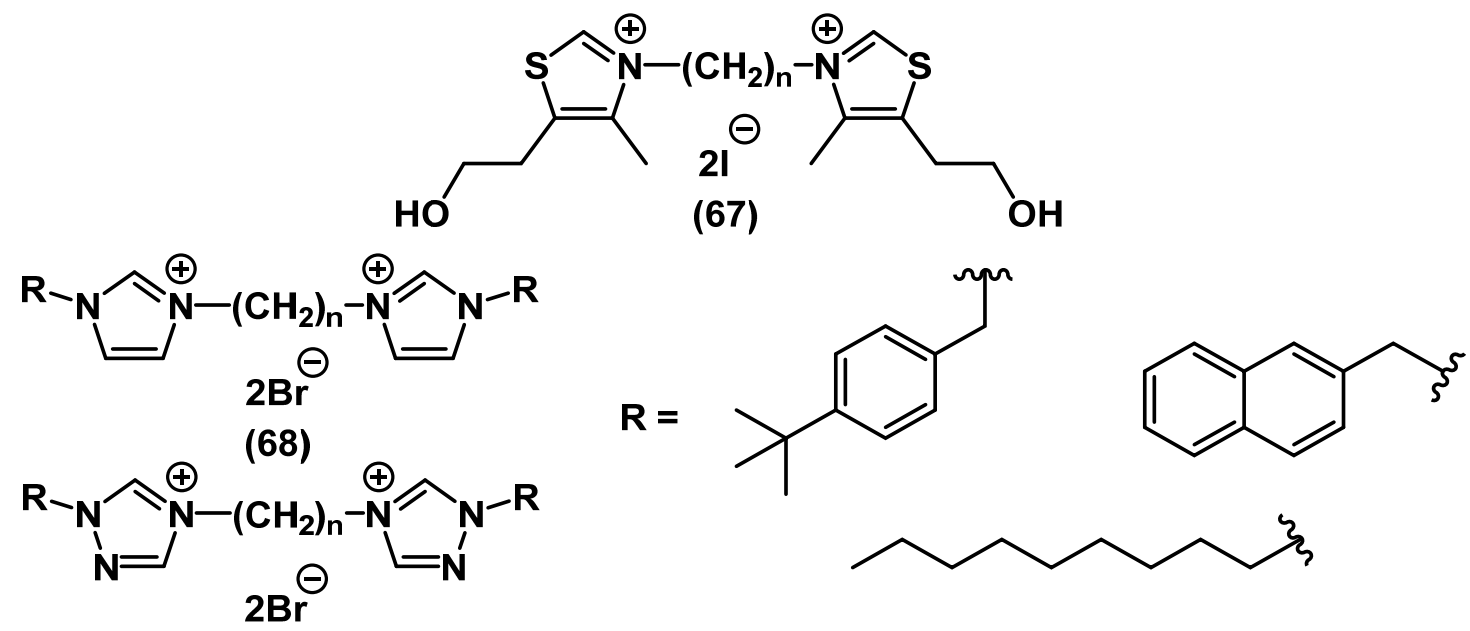

(69)

Figure 24. Bis-QACs containing saturated heterocycles.

Thomas and colleagues synthesized QACs based on bis-thiazole 67 , bis-imidazole 68 and bis-triazole 69 . While thiazole derivatives with an alkyl spacer and without lipophilic tails 67 did not show high activity, bis-QACs with nitrogen heterocycles 68-69 demonstrated MIC values lower than that of CHG [113].

In contrast, in the work of Shirai and colleagues, thiazole bis-QACs with alkyl tails 71 (Figure 25) exhibited a wide spectrum of antibacterial and antifungal effects [114]. This is additional evidence that the tails in the QAC structure are strong inducer of the biological effect against pathogens. Shrestha and colleagues studied the antibacterial and antifungal activity of bis-triazole QAC based on benzoquinone 72 (Figure 25) [115].

Inspired by the success of octenidine on the market of cationic biocides, scientists have begun to actively develop a class of bispyridinium salts with various types of spacers (Figure 26).

In the work of Minbiole and colleagues, bispiridinium QAC derivatives of paraquats 73-75 and bis-QACs without a spacer between pyridinium heads were studied. The activity of meta-75 and parameta-analogs 74 was more pronounced. Cyclovoltamperometric analysis showed the predisposition of paraquats 73 to reversible oxidation-reduction processes and the formation of "superoxide". This presumably increases the toxicity, while metaquats 75 and parametaquats 74 are not subject to this possibility and thus can be less toxic. In addition, given the high activity of parameta-derivatives 74 , this indicates the incoherence between the increase in the biocidal action of QACs and their redox capacity $[116,117]$. A study on the dependence of the activity on the rigidity of the structure for bispyridinium-QACs with alkyl spacers with different saturations 76-78 showed ambiguous results. While this dependence was not observed for QACs with alkyl chains as tails, and the MIC values remained approximately at the same level, in the case 
of bis-QACs with amide bridges in the tails, a sharp decrease in the activity was observed upon increasing the structural rigidity. The authors showed that in such rigid structures, the bis-QAC activity decreased as the charged heads moved away from each other [118].<smiles></smiles>

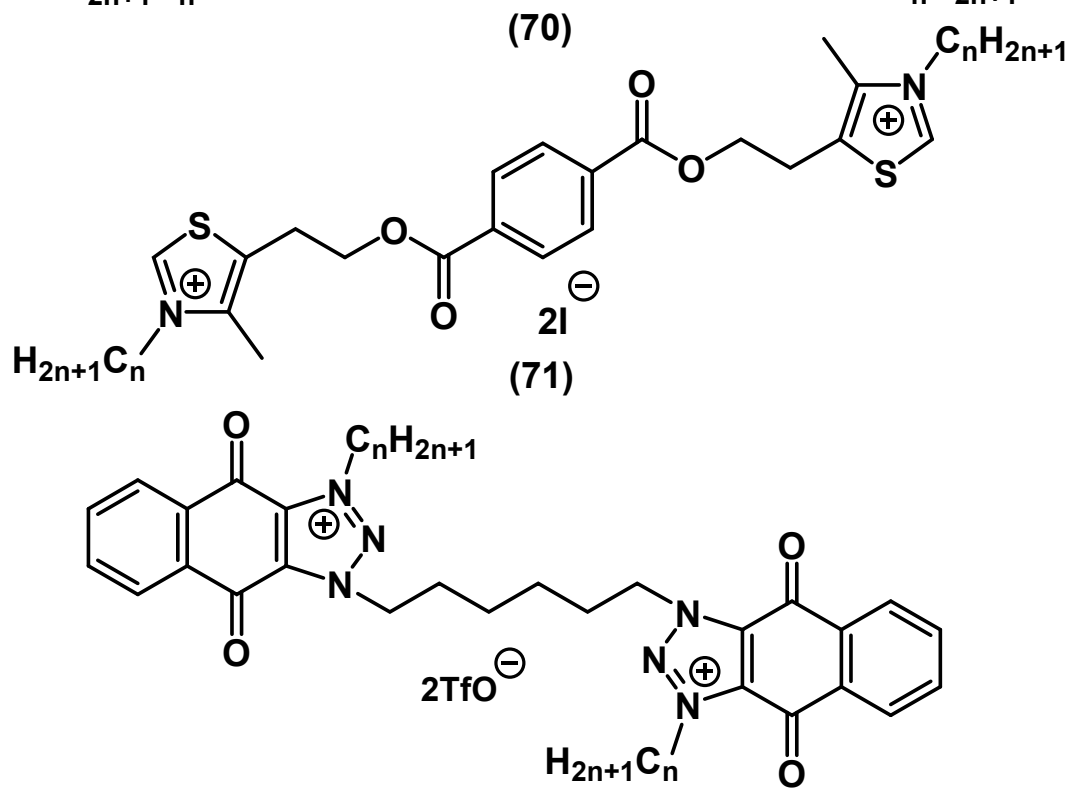

(72)

Figure 25. Bis-QACs containing unsaturated heterocycles.
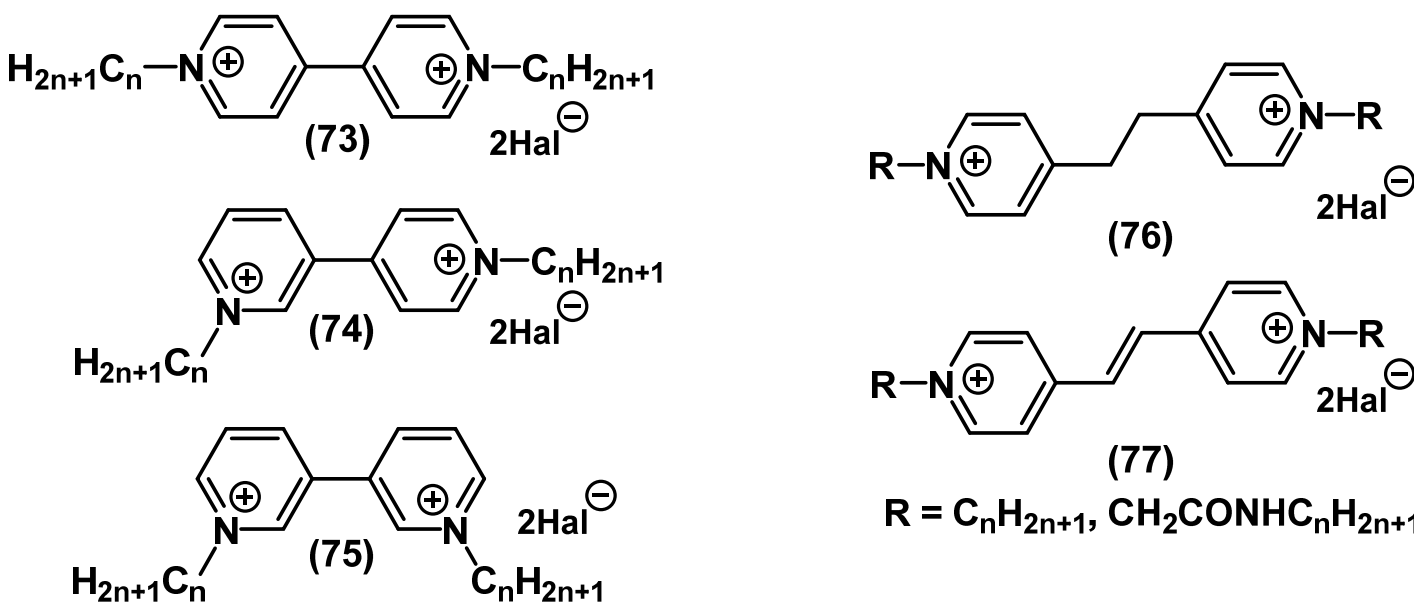

(76)

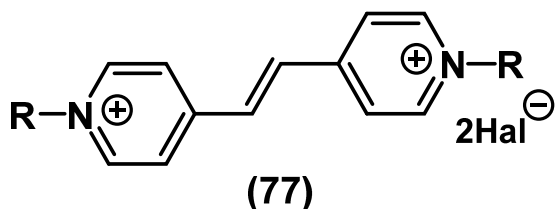

$\mathrm{R}=\mathrm{C}_{\mathrm{n}} \mathrm{H}_{2 \mathrm{n}+1}, \mathrm{CH}_{2} \mathrm{CONHC}_{n} \mathrm{H}_{2 n+1}$

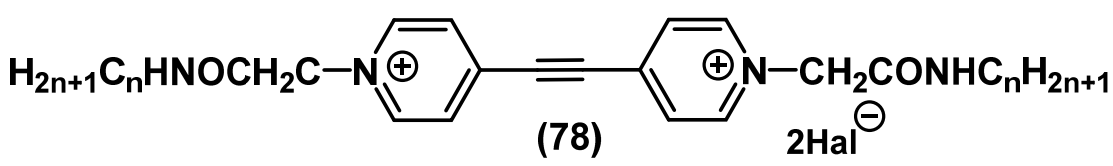

Figure 26. Pyridine-based bis-QACs without spacers and with alkyl spacers.

In the last few years, new biocidal pyridine-based bis-QACs containing an aromatic fragment in a spacer have been synthesized (Figure 27). Thus, bis-QACs with 
1,4-dioxophenyl as spacer $\mathbf{7 9}$ were significantly more active than commercial QACs (BAC 1, CHG 7) [119-121]. Vereshchagin's group studied the dependence of the activity of biocides on the size of the aromatic spacer of salts, as well as the location of the spacer relative to the charged pyridinium nitrogen 79-83 [122-126]. It was discovered that the QAC activity increased upon increasing the length of the aromatic spacer. The activity increased in the following order: mono- $\mathbf{7 9}<$ bi- $\mathbf{8 0}<$ terphenyl $\mathbf{8 2}$ [122,124]. It can be assumed that in such structures, the activity increases with an increase in the distance between the nitrogen atoms. It is worth noting that the optimal length of the alkyl tails also varied in this series: $C_{12}$ for phenyl $\mathbf{7 9}, C_{10}$ for biphenyl $\mathbf{8 0}$, and $C_{8}$ for terphenyl $\mathbf{8 2}$. The influence of the position of substitution in pyridine turned out to be ambiguous. In the case of biphenyl 80, the meta-salts turned out to be slightly more active than the para-derivatives, while the opposite was observed for the more mobile biphenyl ether $\mathbf{8 1}[123,126]$. The ortho-salts showed strikingly lower activity. However, this was not the case for QACs of 2,7-dihydroxynaphthalene derivatives 83 , and the biocidal effect of the orthosalts was extremely high [125]. From the viewpoint of their activity, the leading compounds from the series of bis-QACs with aromatic spacers were superior to the widely used QACs, such as CHG 7, CPC 5, BAC 1, and miramistin 4, and were comparable to OCT 6 (Figure 27).

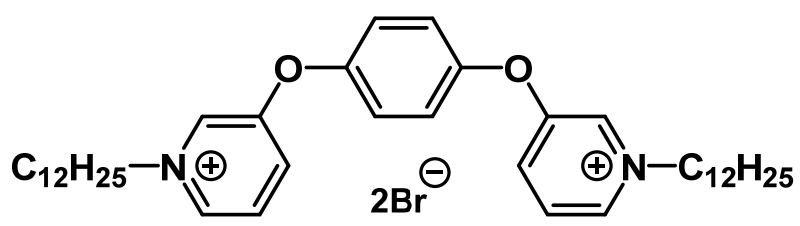

(79)

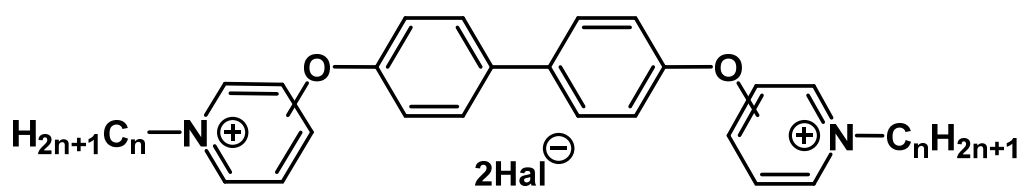

(80)

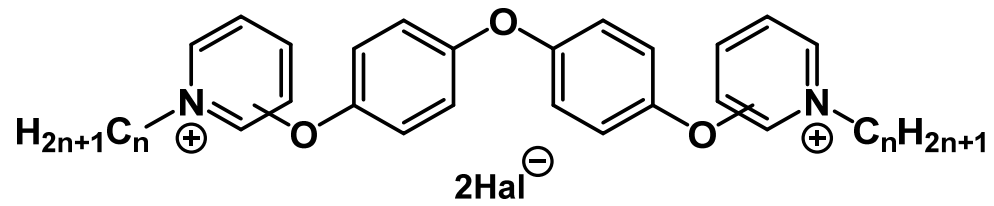

(81)

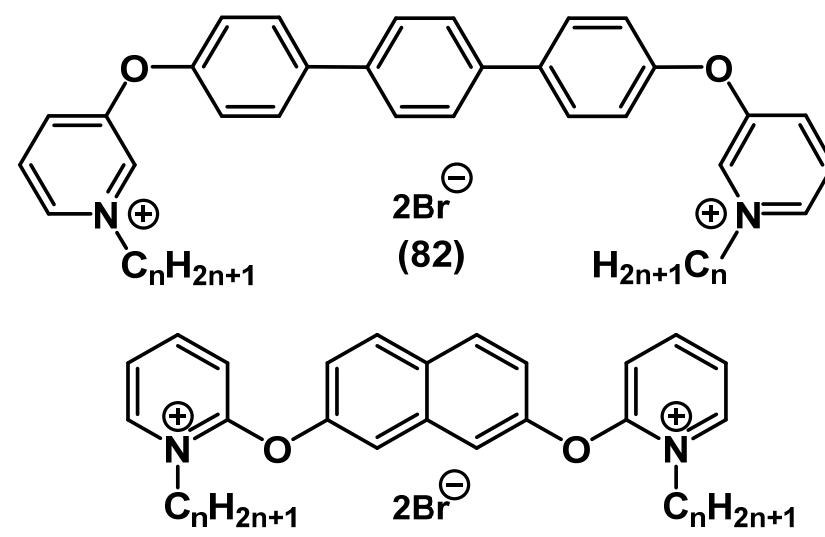

(83)

Figure 27. Pyridine-based bis-QACs containing aromatic spacers.

There is a broad variety of structures of bispyridinium salts containing mixed spacers (Figure 28). 


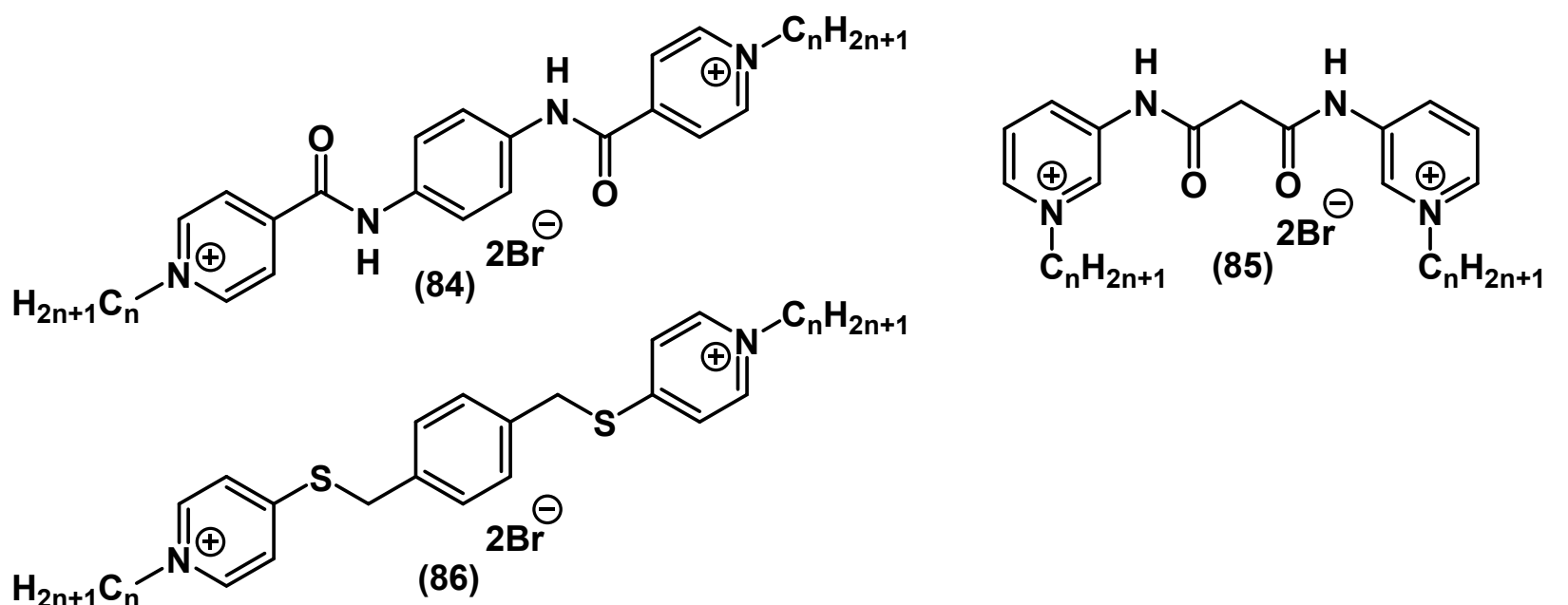

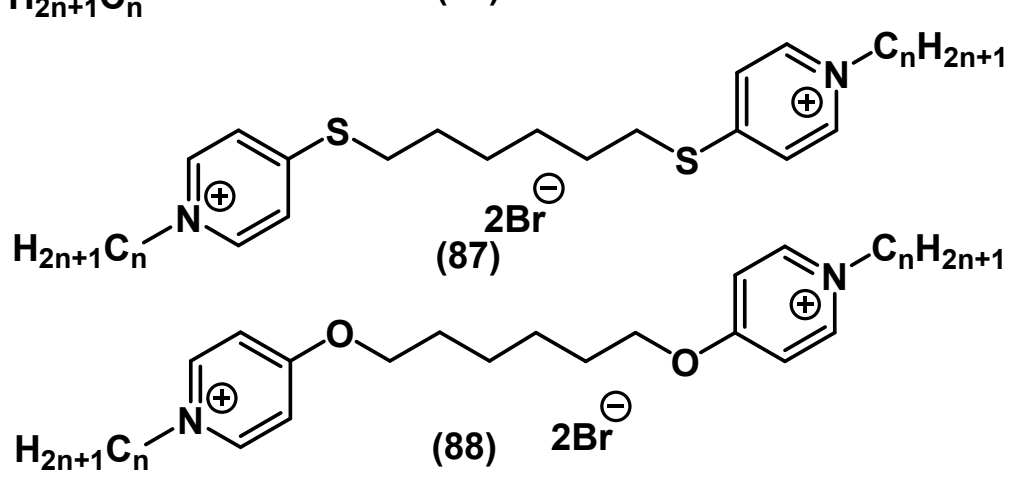

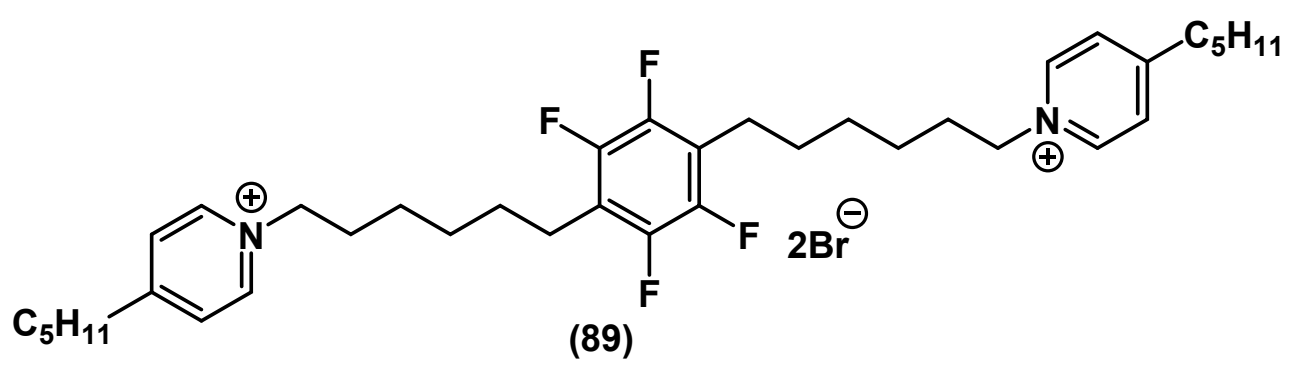

Figure 28. Pyridine-based bis-QACs containing mixed spacers.

Kourai and colleagues initiated studies on bis-pyridine salts 84, 86-88 [127-132]. Later, Obando and colleagues proposed the synthesis of biologically active bis-QACs containing mixed alkyl-aromatic spacers 89 [133]. In their recent investigation, Hao and colleagues performed a comprehensive physical-chemical and biological analysis of bis-QACs with amide bridges 85 [134].

Pentaerythritol-based bis-QACs 90-91 (Figure 29) were developed by Yamamoto and colleagues. These substances revealed a broad scope of antibacterial and antifungal activities [120]. At that time, the substances with condensed hydroxy groups 90 had higher activity than those with free hydroxy groups 91. The biocompatibility of the series leaders was similar to or higher than that of the common antiseptics (BAC, CPC, OCT, PHMB). Furthermore, Vereshchagin presented a synthetic route and microbiological study of pentaerythritol bis-QACs as OCT analogues 92 [135]. The salts were active towards MRSA and E. coli (Figure 29). 

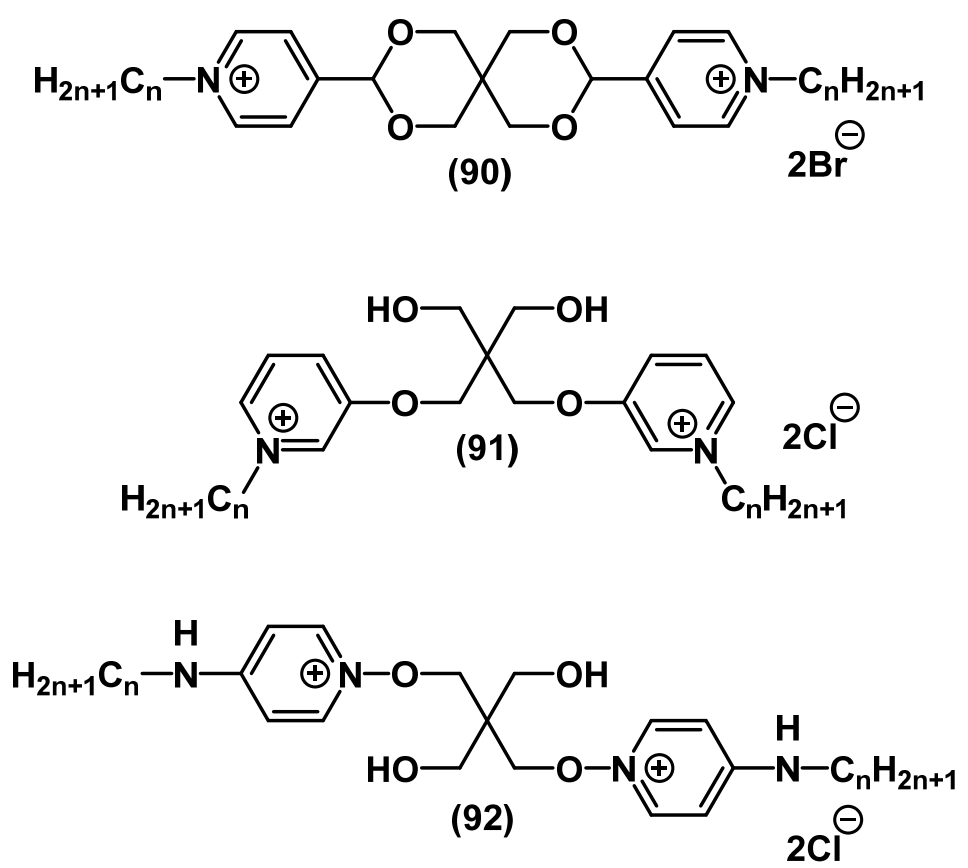

Figure 29. Pyridine-based bis-QACs containing pentaerythritol.

An overview of the antibacterial activity of bis-QACs, analyzed in the review, is shown in Table 4.

Table 4. Antimicrobial activity of Bis-QACs *

\begin{tabular}{|c|c|c|c|c|c|c|}
\hline $\begin{array}{l}\text { Series/ } \\
\text { Compound }\end{array}$ & Strain & $\begin{array}{c}\mathrm{MIC}, \\
\mathrm{mg} \cdot \mathrm{L}^{-1}\end{array}$ & $\begin{array}{c}\mathrm{MBC} \\
\mathrm{mg} \cdot \mathrm{L}^{-1}\end{array}$ & Method & Notes & Ref. \\
\hline \multirow{4}{*}{42} & S. aureus SH1000 & $1 \mu \mathrm{M}$ & & \multirow{4}{*}{ Broth microdilution } & & \multirow{4}{*}{ [75] } \\
\hline & E. faecalis OG1RF & $1 \mu \mathrm{M}$ & & & & \\
\hline & E. coli MC4100 & $2 \mu \mathrm{M}$ & & & & \\
\hline & P. aeruginosa PAO1-WT & $4 \mu \mathrm{M}$ & & & & \\
\hline \multirow{4}{*}{43} & S. aureus SH1000 & $1 \mu \mathrm{M}$ & & \multirow{4}{*}{ Broth microdilution } & & \multirow{4}{*}{ [71] } \\
\hline & E. faecalis OG1RF & $1 \mu \mathrm{M}$ & & & & \\
\hline & E. coli MC4100 & $2 \mu \mathrm{M}$ & & & & \\
\hline & P. aeruginosa PAO1-WT & $4 \mu \mathrm{M}$ & & & & \\
\hline \multirow{4}{*}{44} & S. aureus $\mathrm{SH} 1000$ я & $1 \mu \mathrm{M}$ & & \multirow{4}{*}{ Broth microdilution } & & \multirow{4}{*}{ [71] } \\
\hline & E. faecalis OG1RF & $1 \mu \mathrm{M}$ & & & & \\
\hline & E. coli MC4100 & $1 \mu \mathrm{M}$ & & & & \\
\hline & P. aeruginosa PAO1-WT & $4 \mu \mathrm{M}$ & & & & \\
\hline \multirow{3}{*}{46} & S. aureus Mau 29/58 & $0.4 \mu \mathrm{M}$ & & \multirow{3}{*}{$\begin{array}{l}\text { Suspension } \\
\text { micromethod }\end{array}$} & & \multirow{3}{*}{ [101] } \\
\hline & E. coli $377 / 79$ & $3.1 \mu \mathrm{M}$ & & & & \\
\hline & C. albicans $45 / 54$ & $1.5 \mu \mathrm{M}$ & & & & \\
\hline \multirow{2}{*}{47} & S. aureus & $13 \mu \mathrm{M}$ & & \multirow{2}{*}{ Broth microdilution } & & \multirow{2}{*}{ [103] } \\
\hline & E. coli & $10 \mu \mathrm{M}$ & & & & \\
\hline \multirow{4}{*}{48} & S. aureus SH1000 & 2 & 2 & \multirow{4}{*}{ Broth microdilution } & & \multirow{4}{*}[105]{} \\
\hline & E. faecalis OG1RF & 18 & 18 & & & \\
\hline & E. coli MC4100 & 18 & 18 & & & \\
\hline & P. aeruginosa PAO1-WT & 37 & 37 & & & \\
\hline
\end{tabular}


Table 4. Cont.

\begin{tabular}{|c|c|c|c|c|c|c|}
\hline $\begin{array}{c}\text { Series/ } \\
\text { Compound }\end{array}$ & Strain & $\begin{array}{c}\mathrm{MIC} \\
\mathrm{mg} \cdot \mathrm{L}^{-1}\end{array}$ & $\begin{array}{c}\mathrm{MBC} \\
\mathrm{mg} \cdot \mathrm{L}^{-1}\end{array}$ & Method & Notes & Ref. \\
\hline \multirow{4}{*}{49} & S. aureus SH1000 & 10 & 10 & \multirow{4}{*}{ Broth microdilution } & & \multirow{4}{*}{ [105] } \\
\hline & E. faecalis OG1RF & 18 & 18 & & & \\
\hline & E. coli MC4100 & 37 & 37 & & & \\
\hline & P. aeruginosa PAO1-WT & 149 & 149 & & & \\
\hline \multirow{4}{*}{50} & S. aureus SH1000 & 10 & 10 & \multirow{4}{*}{ Broth microdilution } & & \multirow{4}{*}{ [105] } \\
\hline & E. faecalis OG1RF & 30 & 30 & & & \\
\hline & E. coli MC4100 & 74 & 74 & & & \\
\hline & P. aeruginosa PAO1-WT & 297 & 297 & & & \\
\hline \multirow{4}{*}{51} & S. aureus SH1000 & 4 & 4 & \multirow{4}{*}{ Broth microdilution } & & \multirow{4}{*}{ [105] } \\
\hline & E. faecalis OG1RF & 18 & 18 & & & \\
\hline & E. coli MC4100 & 37 & 37 & & & \\
\hline & P. aeruginosa PAO1-WT & 74 & 74 & & & \\
\hline \multirow{4}{*}{52} & S. aureus SH1000 & 4 & 4 & \multirow{4}{*}{ Broth microdilution } & & \multirow{4}{*}{ [105] } \\
\hline & E. faecalis OG1RF & 10 & 10 & & & \\
\hline & E. coli MC4100 & 18 & 18 & & & \\
\hline & P. aeruginosa PAO1-WT & 74 & 74 & & & \\
\hline \multirow{6}{*}{53} & S. aureus SH1000 & $0.5 \mu \mathrm{M}$ & & \multirow{6}{*}{ Broth microdilution } & & \multirow{6}{*}{ [107] } \\
\hline & MRSA 300-0114 & $1 \mu \mathrm{M}$ & & & & \\
\hline & MRSA ATCC 33592 & $0.25 \mu \mathrm{M}$ & & & & \\
\hline & E. faecalis OG1RF & $0.25 \mu \mathrm{M}$ & & & & \\
\hline & E. coli MC4100 & $1 \mu \mathrm{M}$ & & & & \\
\hline & P. aeruginosa PAO1-WT & $2 \mu \mathrm{M}$ & & & & \\
\hline \multirow{10}{*}{54} & S. aureus ATCC 29213 & 0.5 & & \multirow{10}{*}{ Broth microdilution } & \multirow{10}{*}{$\begin{array}{c}\text { Tested in vivo } \\
\text { with proved } \\
\text { efficiency }\end{array}$} & \multirow{10}{*}{ [106] } \\
\hline & S. epidermidis (clinical) & 2 & & & & \\
\hline & B. subtilis 168 & 1 & & & & \\
\hline & E. coli ATCC 25922 & 0.5 & & & & \\
\hline & K. pneumoniae 1813 & 4 & & & & \\
\hline & P. aeruginosa ATCC 27853 & 0.5 & & & & \\
\hline & T. rubrum 1336 (clinical) & 32 & & & & \\
\hline & A. niger F-1119 & 16 & & & & \\
\hline & C. albicans NCTC- 885-653 & 16 & & & & \\
\hline & F. oxysporum KM-19 (clinical) & 32 & & & & \\
\hline 55 & S. aureus ATCC 29213 & 4 & & Broth microdilution & & [65] \\
\hline \multirow{7}{*}{57} & P. aeruginosa ATCC 27583 & $6.3 \mu \mathrm{M}$ & & \multirow{7}{*}{ Broth microdilution } & & \multirow{7}{*}{ [108] } \\
\hline & P. aeruginosa ATCC 10145 & $5.2 \mu \mathrm{M}$ & & & & \\
\hline & P. aeruginosa ATCC 3080 & $1.6 \mu \mathrm{M}$ & & & & \\
\hline & K. pneumoniae ATCC 4352 & $0.4 \mu \mathrm{M}$ & & & & \\
\hline & K. pneumoniae ATCC 13883 & $0.8 \mu \mathrm{M}$ & & & & \\
\hline & P. vulgaris ATCC 13315 & $0.4 \mu \mathrm{M}$ & & & & \\
\hline & P. mirabilis NBRC 3849 & $6.3 \mu \mathrm{M}$ & & & & \\
\hline
\end{tabular}


Table 4. Cont.

\begin{tabular}{|c|c|c|c|c|c|c|}
\hline $\begin{array}{c}\text { Series/ } \\
\text { Compound }\end{array}$ & Strain & $\begin{array}{c}\mathrm{MIC}^{\prime} \\
\mathrm{mg} \cdot \mathrm{L}^{-1}\end{array}$ & $\begin{array}{c}\mathrm{MBC}, \\
\mathrm{mg} \cdot \mathrm{L}^{-1}\end{array}$ & Method & Notes & Ref. \\
\hline & E. coli K12 W3110 & $0.8 \mu \mathrm{M}$ & & & & \\
\hline & E. coli IFO 3301 & $0.2 \mu \mathrm{M}$ & & & & \\
\hline & E. coli IFO 3972 & $1.3 \mu \mathrm{M}$ & & & & \\
\hline & B. subtilis IFO 3134 & $0.8 \mu \mathrm{M}$ & & & & \\
\hline & B. subtilis ATCC 6633 & $0.8 \mu \mathrm{M}$ & & & & \\
\hline & B. cereus IFO 3001 & $0.4 \mu \mathrm{M}$ & & & & \\
\hline & B. megaterium IFO 3003 & $0.3 \mu \mathrm{M}$ & & & & \\
\hline & S. aureus ATCC 25923 & $0.3 \mu \mathrm{M}$ & & & & \\
\hline & S. aureus IFO 12732 & $0.4 \mu \mathrm{M}$ & & & & \\
\hline & A. niger IFO 6341 & $8 \mu \mathrm{M}$ & & & & \\
\hline & A. niger IFO 6342 & $4 \mu \mathrm{M}$ & & & & \\
\hline & A. niger IFO 4414 & $4 \mu \mathrm{M}$ & & & & \\
\hline & C. globosum IFO 6347 & $8 \mu \mathrm{M}$ & & & & \\
\hline & R. oryzae IFO 31005 & $2 \mu \mathrm{M}$ & & & & \\
\hline & P. citrinum IFO 6352 & $8 \mu \mathrm{M}$ & & & & \\
\hline & A. pullulans IFO 6353 & $16 \mu \mathrm{M}$ & & & & \\
\hline & C. cladosporioides IFO 6348 & $4 \mu \mathrm{M}$ & & & & \\
\hline & G. virens IFO 6355 & $8 \mu \mathrm{M}$ & & & & \\
\hline \multirow{6}{*}{58} & S. aureus SH1000 & $1 \mu \mathrm{M}$ & & \multirow{6}{*}{ Broth microdilution } & & \multirow{6}{*}{ [109] } \\
\hline & MRSA 300-0114 & $1 \mu \mathrm{M}$ & & & & \\
\hline & MRSA ATCC 33592 & $2 \mu \mathrm{M}$ & & & & \\
\hline & E. faecalis OG1RF & $8 \mu \mathrm{M}$ & & & & \\
\hline & E. coli MC4100 & $8 \mu \mathrm{M}$ & & & & \\
\hline & P. aeruginosa PAO1-WT & $8 \mu \mathrm{M}$ & & & & \\
\hline \multirow{6}{*}{59} & S. aureus SH1000 & $0.25 \mu \mathrm{M}$ & & \multirow{6}{*}{ Broth microdilution } & & \multirow{6}{*}{ [109] } \\
\hline & MRSA 300-0114 & $2 \mu \mathrm{M}$ & & & & \\
\hline & MRSA ATCC 33592 & $0.5 \mu \mathrm{M}$ & & & & \\
\hline & E. faecalis OG1RF & $4 \mu \mathrm{M}$ & & & & \\
\hline & E. coli MC4100 & $2 \mu \mathrm{M}$ & & & & \\
\hline & P. aeruginosa PAO1-WT & $8 \mu \mathrm{M}$ & & & & \\
\hline \multirow{3}{*}{60} & S. aureus ATCC 25923 & 64 & 128 & \multirow{3}{*}{ Broth microdilution } & \multirow{3}{*}{ Surfactant } & \multirow{3}{*}{ [110] } \\
\hline & B. subtilis ATCC 6633 & 16 & 32 & & & \\
\hline & E. coli ATCC 25922 & 16 & 64 & & & \\
\hline \multirow{6}{*}{61} & S. aureus SH1000 & $1 \mu \mathrm{M}$ & & \multirow{6}{*}{ Broth microdilution } & \multirow{6}{*}{$\begin{array}{c}\text { Natural } \\
\text { derivatives }\end{array}$} & \multirow{6}{*}[74]{} \\
\hline & MRSA 300-0114 & $4 \mu \mathrm{M}$ & & & & \\
\hline & MRSA ATCC 33592 & $2 \mu \mathrm{M}$ & & & & \\
\hline & E. faecalis OG1RF & $2 \mu \mathrm{M}$ & & & & \\
\hline & E. coli MC4100 & $4 \mu \mathrm{M}$ & & & & \\
\hline & P. aeruginosa PAO1-WT & $32 \mu \mathrm{M}$ & & & & \\
\hline
\end{tabular}


Table 4. Cont.

\begin{tabular}{|c|c|c|c|c|c|c|}
\hline $\begin{array}{c}\text { Series/ } \\
\text { Compound }\end{array}$ & Strain & $\begin{array}{c}\mathrm{MIC} \\
\mathrm{mg} \cdot \mathrm{L}^{-1}\end{array}$ & $\begin{array}{c}\mathrm{MBC} \\
\mathrm{mg} \cdot \mathrm{L}^{-1}\end{array}$ & Method & Notes & Ref. \\
\hline \multirow{6}{*}{62} & S. aureus SH1000 & $1 \mu \mathrm{M}$ & & \multirow{6}{*}{ Broth microdilution } & \multirow{6}{*}{$\begin{array}{c}\text { Natural } \\
\text { derivatives }\end{array}$} & \multirow{6}{*}{ [74] } \\
\hline & MRSA 300-0114 & $1 \mu \mathrm{M}$ & & & & \\
\hline & MRSA ATCC 33592 & $1 \mu \mathrm{M}$ & & & & \\
\hline & E. faecalis OG1RF & $2 \mu \mathrm{M}$ & & & & \\
\hline & E. coli MC4100 & $2 \mu \mathrm{M}$ & & & & \\
\hline & P. aeruginosa PAO1-WT & $8 \mu \mathrm{M}$ & & & & \\
\hline \multirow{6}{*}{63} & S. aureus SH1000 & $2 \mu \mathrm{M}$ & & \multirow{6}{*}{ Broth microdilution } & & \multirow{6}{*}{ [111] } \\
\hline & MRSA 300-0114 & $1 \mu \mathrm{M}$ & & & & \\
\hline & MRSA ATCC 33592 & $2 \mu \mathrm{M}$ & & & & \\
\hline & E. faecalis OG1RF & $4 \mu \mathrm{M}$ & & & & \\
\hline & E. coli MC4100 & $1 \mu \mathrm{M}$ & & & & \\
\hline & P. aeruginosa PAO1-WT & $4 \mu \mathrm{M}$ & & & & \\
\hline \multirow{6}{*}{64} & S. aureus SH1000 & $2 \mu \mathrm{M}$ & & \multirow{6}{*}{ Broth microdilution } & & \multirow{6}{*}{ [111] } \\
\hline & MRSA 300-0114 & $2 \mu \mathrm{M}$ & & & & \\
\hline & MRSA ATCC 33592 & $2 \mu \mathrm{M}$ & & & & \\
\hline & E. faecalis OG1RF & $4 \mu \mathrm{M}$ & & & & \\
\hline & E. coli MC4100 & $2 \mu \mathrm{M}$ & & & & \\
\hline & P. aeruginosa PAO1-WT & $4 \mu \mathrm{M}$ & & & & \\
\hline \multirow{4}{*}{65} & S. aureus SH1000 & $0.5 \mu \mathrm{M}$ & & \multirow{4}{*}{ Broth microdilution } & & \multirow{4}{*}{ [112] } \\
\hline & MRSA 300-0114 & $0.5 \mu \mathrm{M}$ & & & & \\
\hline & E. coli MC4100 & $1 \mu \mathrm{M}$ & & & & \\
\hline & P. aeruginosa PAO1-WT & $2 \mu \mathrm{M}$ & & & & \\
\hline \multirow{3}{*}{66} & S. aureus SH1000 & $0.5 \mu \mathrm{M}$ & & \multirow{3}{*}{ Broth microdilution } & & \multirow{3}{*}{ [72] } \\
\hline & MRSA 300-0114 & $0.5 \mu \mathrm{M}$ & & & & \\
\hline & MRSA ATCC 33592 & $0.5 \mu \mathrm{M}$ & & & & \\
\hline \multirow{4}{*}{67} & S. aureus ATCC 29213 & 16 & & \multirow{4}{*}{ Broth microdilution } & & \multirow{4}{*}{ [113] } \\
\hline & E. faecalis ATCC 29212 & 64 & & & & \\
\hline & E. coli ATCC 25922 & 128 & & & & \\
\hline & P. aeruginosa ATCC 27853 & 256 & & & & \\
\hline \multirow{8}{*}{68} & S. aureus ATCC 29213 & 0.25 & & \multirow{8}{*}{ Broth microdilution } & & \multirow{8}{*}{ [113] } \\
\hline & $\operatorname{MRSA}(m e c A)$ & 0.5 & & & & \\
\hline & E. faecalis ATCC 29212 & 0.5 & & & & \\
\hline & $\begin{array}{l}\text { Vancomycin-resistant } E \text {. } \\
\text { faecalis (van } A)\end{array}$ & 0.5 & & & & \\
\hline & E. coli ATCC 25922 & 0.5 & & & & \\
\hline & $\begin{array}{c}\text { Extended-spectrum } \\
\text { b-lactamase-producing } \\
\text { E. coli }\end{array}$ & 1 & & & & \\
\hline & P. aeruginosa ATCC 27853 & 4 & & & & \\
\hline & $\begin{array}{l}\text { P. aeruginosa resistant, } \\
\text { efflux pump }\end{array}$ & 8 & & & & \\
\hline
\end{tabular}


Table 4. Cont.

\begin{tabular}{|c|c|c|c|c|c|c|}
\hline $\begin{array}{c}\text { Series/ } \\
\text { Compound }\end{array}$ & Strain & $\begin{array}{c}\mathrm{MIC}_{,} \\
\mathrm{mg} \cdot \mathrm{L}^{-1}\end{array}$ & $\begin{array}{c}\mathrm{MBC}, \\
\mathrm{mg} \cdot \mathrm{L}^{-1}\end{array}$ & Method & Notes & Ref. \\
\hline \multirow{8}{*}{69} & S. aureus ATCC 29213 & 0.5 & & \multirow{8}{*}{ Broth microdilution } & & \multirow{8}{*}{ [113] } \\
\hline & $\operatorname{MRSA}(m e c A)$ & 0.5 & & & & \\
\hline & E. faecalis ATCC 29212 & 0.5 & & & & \\
\hline & $\begin{array}{l}\text { Vancomycin-resistant } \\
\text { E. faecalis (vanA) }\end{array}$ & 0.5 & & & & \\
\hline & E. coli ATCC 25922 & 0.5 & & & & \\
\hline & $\begin{array}{c}\text { Extended-spectrum } \\
\text { b-lactamase-producing } \\
\text { E. coli }\end{array}$ & 1 & & & & \\
\hline & P. aeruginosa ATCC 27853 & 2 & & & & \\
\hline & $\begin{array}{l}\text { P. aeruginosa resistant, } \\
\text { efflux pump }\end{array}$ & 2 & & & & \\
\hline \multirow{10}{*}{70} & P. aeruginosa ATCC 27853 & $17 \mu \mathrm{M}$ & & \multirow{10}{*}{ Broth microdilution } & & \multirow{10}{*}{ [114] } \\
\hline & K. pneumoniae ATCC 4352 & $2.1 \mu \mathrm{M}$ & & & & \\
\hline & P. mirabilis NBRC 3849 & $3.1 \mu \mathrm{M}$ & & & & \\
\hline & E. coli IFO 12713 & $1.6 \mu \mathrm{M}$ & & & & \\
\hline & S. marcescens ATCC 13880 & $3.1 \mu \mathrm{M}$ & & & & \\
\hline & M. luteus IFO 12708 & $0.65 \mu \mathrm{M}$ & & & & \\
\hline & B. subtilis ATCC 6633 & $0.91 \mu \mathrm{M}$ & & & & \\
\hline & B. cereus IFO 3001 & $1.6 \mu \mathrm{M}$ & & & & \\
\hline & S. aureus IFO 12732 & $0.23 \mu \mathrm{M}$ & & & & \\
\hline & MRSA COL 1 & $1.6 \mu \mathrm{M}$ & & & & \\
\hline \multirow{10}{*}{71} & P. aeruginosa ATCC 27853 & $13 \mu \mathrm{M}$ & & \multirow{10}{*}{ Broth microdilution } & & \multirow{10}{*}{ [114] } \\
\hline & K. pneumoniae ATCC 4352 & $1.6 \mu \mathrm{M}$ & & & & \\
\hline & P. mirabilis NBRC 3849 & $5.2 \mu \mathrm{M}$ & & & & \\
\hline & E. coli IFO 12713 & $1.6 \mu \mathrm{M}$ & & & & \\
\hline & S. marcescens ATCC 13880 & $6.3 \mu \mathrm{M}$ & & & & \\
\hline & M. luteus IFO 12708 & $0.78 \mu \mathrm{M}$ & & & & \\
\hline & B. subtilis ATCC 6633 & $1.0 \mu \mathrm{M}$ & & & & \\
\hline & B. cereus IFO 3001 & $1.3 \mu \mathrm{M}$ & & & & \\
\hline & S. aureus IFO 12732 & $0.33 \mu \mathrm{M}$ & & & & \\
\hline & MRSA COL 1 & $1.3 \mu \mathrm{M}$ & & & & \\
\hline \multirow{11}{*}{72} & S. aureus ATCC 25923 & 4 & & \multirow{11}{*}{ Broth microdilution } & & \multirow{11}{*}{ [115] } \\
\hline & MRSA ATCC 33591 & 4 & & & & \\
\hline & E. faecalis ATCC 1299 & 1 & & & & \\
\hline & E. coli ATCC 25922 & 2 & & & & \\
\hline & P. aeruginosa ATCC 27853 & 4 & & & & \\
\hline & K. pneumoniae ATCC 13883 & 16 & & & & \\
\hline & A. flavus & 15.63 & & & & \\
\hline & C. albicans 64124 & 3.91 & & & & \\
\hline & C. albicans MYA2876 & 3.91 & & & & \\
\hline & C. neoformans & 3.9 & & & & \\
\hline & R. pilimanae & 2.0 & & & & \\
\hline
\end{tabular}


Table 4. Cont.

\begin{tabular}{|c|c|c|c|c|c|c|}
\hline $\begin{array}{c}\text { Series/ } \\
\text { Compound }\end{array}$ & Strain & $\begin{array}{c}\mathrm{MIC}^{\prime} \\
\mathrm{mg} \cdot \mathrm{L}^{-1}\end{array}$ & $\begin{array}{c}\mathrm{MBC}, \\
\mathrm{mg} \cdot \mathrm{L}^{-1}\end{array}$ & Method & Notes & Ref. \\
\hline \multirow{4}{*}{73} & S. aureus SH1000 & $2 \mu \mathrm{M}$ & & \multirow{4}{*}{ Broth microdilution } & & \multirow{4}{*}{ [117] } \\
\hline & E. faecalis OG1RF & $2 \mu \mathrm{M}$ & & & & \\
\hline & E. coli MC4100 & $2 \mu \mathrm{M}$ & & & & \\
\hline & P. aeruginosa PAO1-WT & $16 \mu \mathrm{M}$ & & & & \\
\hline \multirow{4}{*}{74} & S. aureus SH1000 & $0.5 \mu \mathrm{M}$ & & \multirow{4}{*}{ Broth microdilution } & & \multirow{4}{*}{ [117] } \\
\hline & E. faecalis OG1RF & $0.5 \mu \mathrm{M}$ & & & & \\
\hline & E. coli MC4100 & $0.5 \mu \mathrm{M}$ & & & & \\
\hline & P. aeruginosa PAO1-WT & $1 \mu \mathrm{M}$ & & & & \\
\hline \multirow{4}{*}{75} & S. aureus SH1000 & $0.5 \mu \mathrm{M}$ & & \multirow{4}{*}{ Broth microdilution } & & \multirow{4}{*}{ [117] } \\
\hline & E. faecalis OG1RF & $1 \mu \mathrm{M}$ & & & & \\
\hline & E. coli MC4100 & $1 \mu \mathrm{M}$ & & & & \\
\hline & P. aeruginosa PAO1-WT & $2 \mu \mathrm{M}$ & & & & \\
\hline \multirow{6}{*}{76} & S. aureus SH1000 & $1 \mu \mathrm{M}$ & & \multirow{6}{*}{ Broth microdilution } & & \multirow{6}{*}{ [118] } \\
\hline & MRSA 300-0114 & $1 \mu \mathrm{M}$ & & & & \\
\hline & MRSA ATCC 33592 & $1 \mu \mathrm{M}$ & & & & \\
\hline & E. faecalis OG1RF & $4 \mu \mathrm{M}$ & & & & \\
\hline & E. coli MC4100 & $1 \mu \mathrm{M}$ & & & & \\
\hline & P. aeruginosa PAO1-WT & $4 \mu \mathrm{M}$ & & & & \\
\hline \multirow{6}{*}{77} & S. aureus SH1000 & $1 \mu \mathrm{M}$ & & \multirow{6}{*}{ Broth microdilution } & & \multirow{6}{*}{ [118] } \\
\hline & MRSA 300-0114 & $0.5 \mu \mathrm{M}$ & & & & \\
\hline & MRSA ATCC 33592 & $2 \mu \mathrm{M}$ & & & & \\
\hline & E. faecalis OG1RF & $2 \mu \mathrm{M}$ & & & & \\
\hline & E. coli MC4100 & $1 \mu \mathrm{M}$ & & & & \\
\hline & P. aeruginosa PAO1-WT & $2 \mu \mathrm{M}$ & & & & \\
\hline \multirow{6}{*}{78} & S. aureus SH1000 & $16 \mu \mathrm{M}$ & & \multirow{6}{*}{ Broth microdilution } & & \multirow{6}{*}{ [118] } \\
\hline & MRSA 300-0114 & $32 \mu \mathrm{M}$ & & & & \\
\hline & MRSA ATCC 33592 & $16 \mu \mathrm{M}$ & & & & \\
\hline & E. faecalis OG1RF & $63 \mu \mathrm{M}$ & & & & \\
\hline & E. coli MC4100 & $32 \mu \mathrm{M}$ & & & & \\
\hline & P. aeruginosa PAO1-WT & $63 \mu \mathrm{M}$ & & & & \\
\hline \multirow{7}{*}{79} & MRSA ATCC 43300 & 0.25 & & \multirow{7}{*}{ Broth microdilution } & & \multirow{7}{*}{ [119] } \\
\hline & E. coli ATCC 25922 & 4 & & & & \\
\hline & K. pneumoniae ATCC 700603 & 16 & & & & \\
\hline & A. baumannii ATCC 19606 & 4 & & & & \\
\hline & P. aeruginosa ATCC 27853 & 8 & & & & \\
\hline & C. albicans ATCC 90028 & 0.25 & & & & \\
\hline & C. neoformans ATCC 208821 & 0.25 & & & & \\
\hline
\end{tabular}


Table 4. Cont.

\begin{tabular}{|c|c|c|c|c|c|c|}
\hline $\begin{array}{c}\text { Series/ } \\
\text { Compound }\end{array}$ & Strain & $\begin{array}{c}\mathrm{MIC} \\
\mathrm{mg} \cdot \mathrm{L}^{-1}\end{array}$ & $\begin{array}{c}\mathrm{MBC} \\
\mathrm{mg} \cdot \mathrm{L}^{-1}\end{array}$ & Method & Notes & Ref. \\
\hline \multirow{7}{*}{80} & MRSA ATCC 43300 & 0.25 & & \multirow{7}{*}{ Broth microdilution } & & \multirow{7}{*}{$\begin{array}{l}{[122,} \\
126]\end{array}$} \\
\hline & E. coli ATCC 25922 & 1 & & & & \\
\hline & K. pneumoniae АТСС 700603 & 8 & & & & \\
\hline & A. baumannii ATCC 19606 & 2 & & & & \\
\hline & P. aeruginosa ATCC 27853 & 4 & & & & \\
\hline & C. albicans ATCC 90028 & 0.25 & & & & \\
\hline & C. neoformans ATCC 208821 & 0.25 & & & & \\
\hline \multirow{7}{*}{81} & MRSA ATCC 43300 & 0.25 & & \multirow{7}{*}{ Broth microdilution } & & \multirow{7}{*}{$\begin{array}{l}{[123,} \\
126]\end{array}$} \\
\hline & E. coli ATCC 25922 & 0.25 & & & & \\
\hline & K. pneumoniae ATCC 700603 & 0.25 & & & & \\
\hline & A. baumannii ATCC 19606 & 0.25 & & & & \\
\hline & P. aeruginosa ATCC 27853 & 0.25 & & & & \\
\hline & C. albicans ATCC 90028 & 0.25 & & & & \\
\hline & C. neoformans ATCC 208821 & 4 & & & & \\
\hline \multirow{7}{*}{82} & MRSA ATCC 43300 & 0.25 & & \multirow{7}{*}{ Broth microdilution } & & \multirow{7}{*}{ [124] } \\
\hline & E. coli ATCC 25922 & 0.25 & & & & \\
\hline & K. pneumoniae ATCC 700603 & 16 & & & & \\
\hline & A. baumannii ATCC 19606 & 0.25 & & & & \\
\hline & P. aeruginosa ATCC 27853 & 0.25 & & & & \\
\hline & C. albicans ATCC 90028 & 0.25 & & & & \\
\hline & C. neoformans ATCC 208821 & 0.25 & & & & \\
\hline \multirow{7}{*}{83} & MRSA ATCC 43300 & 0.25 & & \multirow{7}{*}{ Broth microdilution } & & \multirow{7}{*}{ [125] } \\
\hline & E. coli ATCC 25922 & 0.25 & & & & \\
\hline & K. pneuтопiаe АТСС 700603 & 0.25 & & & & \\
\hline & A. baumannii ATCC 19606 & 8 & & & & \\
\hline & P. aeruginosa ATCC 27853 & 0.25 & & & & \\
\hline & C. albicans ATCC 90028 & 0.25 & & & & \\
\hline & C. neoformans ATCC 208821 & 0.25 & & & & \\
\hline \multirow{8}{*}{84} & P. aeruginosa ATCC 27583 & & $6.3 \mu \mathrm{M}$ & \multirow{8}{*}{ Broth microdilution } & & \multirow{8}{*}{ [127] } \\
\hline & K. pneumoniae ATCC 13883 & & $3.1 \mu \mathrm{M}$ & & & \\
\hline & P. mirabilis IFO 3849 & & $6.3 \mu \mathrm{M}$ & & & \\
\hline & E. coli K12 W3110 & & $3.1 \mu \mathrm{M}$ & & & \\
\hline & M. luteus IFO 12708 & & $0.78 \mu \mathrm{M}$ & & & \\
\hline & B. cereus IFO 3001 & & $3.1 \mu \mathrm{M}$ & & & \\
\hline & S. aureus IFO 12732 & & $0.39 \mu \mathrm{M}$ & & & \\
\hline & MRSA IID 1677 & & $3.1 \mu \mathrm{M}$ & & & \\
\hline
\end{tabular}


Table 4. Cont

\begin{tabular}{|c|c|c|c|c|c|c|}
\hline $\begin{array}{c}\text { Series/ } \\
\text { Compound }\end{array}$ & Strain & $\begin{array}{c}\mathrm{MIC}^{\prime} \\
\mathrm{mg} \cdot \mathrm{L}^{-1}\end{array}$ & $\begin{array}{c}\mathrm{MBC} \\
\mathrm{mg} \cdot \mathrm{L}^{-1}\end{array}$ & Method & Notes & Ref. \\
\hline & P. funiculosam IFO 6345 & $1.6 \mu \mathrm{M}$ & & & & \\
\hline & C. globosum IFO 6347 & $3.1 \mu \mathrm{M}$ & & & & \\
\hline & A. pullulans IFO 6353 & $6.3 \mu \mathrm{M}$ & & & & \\
\hline & R. stolonifera IFO 4781 & $25 \mu \mathrm{M}$ & & & & \\
\hline & A. terreus IFO 6346 & $25 \mu \mathrm{M}$ & & & & \\
\hline & A. niger IFO 6342 & $12.5 \mu \mathrm{M}$ & & & & \\
\hline 85 & E. coli & 2.7 & & Broth microdilution & & [134] \\
\hline \multirow{14}{*}{86} & P. aeruginosa ATCC 27583 & & $13 \mu \mathrm{M}$ & \multirow{14}{*}{ Broth microdilution } & & \multirow{14}{*}{ [127] } \\
\hline & K. pneumoniae ATCC 13883 & & $1.6 \mu \mathrm{M}$ & & & \\
\hline & P. mirabilis IFO 3849 & & $13 \mu \mathrm{M}$ & & & \\
\hline & E. coli K12 W3110 & & $6.3 \mu \mathrm{M}$ & & & \\
\hline & M. luteus IFO 12708 & & $0.39 \mu \mathrm{M}$ & & & \\
\hline & B. cereus IFO 3001 & & $1.6 \mu \mathrm{M}$ & & & \\
\hline & S. aureus IFO 12732 & & $0.39 \mu \mathrm{M}$ & & & \\
\hline & MRSA IID 1677 & & $6.3 \mu \mathrm{M}$ & & & \\
\hline & P. funiculosam IFO 6345 & $1.6 \mu \mathrm{M}$ & & & & \\
\hline & C. globosum IFO 6347 & $0.78 \mu \mathrm{M}$ & & & & \\
\hline & A. pullulans IFO 6353 & $6.3 \mu \mathrm{M}$ & & & & \\
\hline & R. stolonifera IFO 4781 & $25 \mu \mathrm{M}$ & & & & \\
\hline & A. terreus IFO 6346 & $12.5 \mu \mathrm{M}$ & & & & \\
\hline & A. niger IFO 6342 & $6.3 \mu \mathrm{M}$ & & & & \\
\hline \multirow{14}{*}{87} & P. aeruginosa ATCC 27583 & & $25 \mu \mathrm{M}$ & \multirow{14}{*}{ Broth microdilution } & & \multirow{14}{*}{ [132] } \\
\hline & K. pneumoniae ATCC 13883 & & $1.6 \mu \mathrm{M}$ & & & \\
\hline & P. mirabilis IFO 3849 & & $13 \mu \mathrm{M}$ & & & \\
\hline & E. coli K12 W3110 & & $6.3 \mu \mathrm{M}$ & & & \\
\hline & M. luteus IFO 12708 & & $0.78 \mu \mathrm{M}$ & & & \\
\hline & B. cereus IFO 3001 & & $3.1 \mu \mathrm{M}$ & & & \\
\hline & S. aureus IFO 12732 & & $0.39 \mu \mathrm{M}$ & & & \\
\hline & MRSA IID 1677 & & $6.3 \mu \mathrm{M}$ & & & \\
\hline & P. funiculosum IFO 6345 & $0.78 \mu \mathrm{M}$ & & & & \\
\hline & C. globosum IFO 6347 & $0.78 \mu \mathrm{M}$ & & & & \\
\hline & A. pullulans IFO 6353 & $3.1 \mu \mathrm{M}$ & & & & \\
\hline & R. stolonifera IFO 4781 & $6.3 \mu \mathrm{M}$ & & & & \\
\hline & A. terreus IFO 6346 & $1.6 \mu \mathrm{M}$ & & & & \\
\hline & A. niger IFO 6342 & $6.3 \mu \mathrm{M}$ & & & & \\
\hline
\end{tabular}


Table 4. Cont.

\begin{tabular}{|c|c|c|c|c|c|c|}
\hline $\begin{array}{c}\text { Series/ } \\
\text { Compound }\end{array}$ & Strain & $\begin{array}{c}\mathrm{MIC}^{\prime} \\
\mathrm{mg} \cdot \mathrm{L}^{-1}\end{array}$ & $\begin{array}{c}\mathrm{MBC}, \\
\mathrm{mg} \cdot \mathrm{L}^{-1}\end{array}$ & Method & Notes & Ref. \\
\hline \multirow{21}{*}{88} & P. aeruginosa ATCC 27583 & $6.3 \mu \mathrm{M}$ & & \multirow{21}{*}{ Broth microdilution } & & \multirow{21}{*}{ [129] } \\
\hline & P. aeruginosa ATCC 10145 & $8.3 \mu \mathrm{M}$ & & & & \\
\hline & K. pneumoniae ATCC 4352 & $1.0 \mu \mathrm{M}$ & & & & \\
\hline & P. rettgeri NIH 96 & $2.1 \mu \mathrm{M}$ & & & & \\
\hline & P. mirabilis IFO 3849 & $25 \mu \mathrm{M}$ & & & & \\
\hline & E. coli IFO 12713 & $1.8 \mu \mathrm{M}$ & & & & \\
\hline & S. enteritidis IFO 3313 & $1.3 \mu \mathrm{M}$ & & & & \\
\hline & B. subtilis IFO 3134 & $0.57 \mu \mathrm{M}$ & & & & \\
\hline & B. subtilis ATCC 6633 & $1.0 \mu \mathrm{M}$ & & & & \\
\hline & B. cereus IFO 3001 & $3.1 \mu \mathrm{M}$ & & & & \\
\hline & S. aureus IFO 12732 & $0.46 \mu \mathrm{M}$ & & & & \\
\hline & MRSA IID 1677 & $1.1 \mu \mathrm{M}$ & & & & \\
\hline & M. luteus IFO 12708 & $0.26 \mu \mathrm{M}$ & & & & \\
\hline & A. niger IFO 6342 & $25 \mu \mathrm{M}$ & & & & \\
\hline & A. niger TSY 0013 & $13 \mu \mathrm{M}$ & & & & \\
\hline & A. pullulans IFO 6353 & $3.1 \mu \mathrm{M}$ & & & & \\
\hline & P. citrinum IFO 6345 & $25 \mu \mathrm{M}$ & & & & \\
\hline & P. funiculosum IFO 6345 & $8.3 \mu \mathrm{M}$ & & & & \\
\hline & R. oryzae IFO 31005 & $13 \mu \mathrm{M}$ & & & & \\
\hline & T. viride IFO 30498 & $25 \mu \mathrm{M}$ & & & & \\
\hline & C. albicans IFO 1061 & $29 \mu \mathrm{M}$ & & & & \\
\hline \multirow{3}{*}{89} & C. neoformans ATCC 90112 & $1.3 \mu \mathrm{M}$ & & \multirow{3}{*}{ Broth microdilution } & & \multirow{3}{*}[133]{} \\
\hline & C. albicans ATCC 10231 & $1.3 \mu \mathrm{M}$ & & & & \\
\hline & A. fumigatus ATCC 204305 & $88 \mu \mathrm{M}$ & & & & \\
\hline \multirow{10}{*}{90} & E. coli ATCC 25922 & 8 & 18 & \multirow{10}{*}{ Broth microdilution } & & \multirow{10}{*}{ [120] } \\
\hline & P. aeruginosa ATCC 6538 & 32 & 8.3 & & & \\
\hline & S. aureus ATCC 278530 & 2.3 & 8.3 & & & \\
\hline & A. baumannii JCM 6841 & 11 & & & & \\
\hline & B. cepacia JCM 5964 & 19 & & & & \\
\hline & E. hirae ATCC 10541 & 5.3 & & & & \\
\hline & E. faecalis ATCC 29212 & 6.7 & & & & \\
\hline & MRSA ATCC 700698 & 11 & & & & \\
\hline & S. epidermidis ATCC 12228 & 5.3 & & & & \\
\hline & C. albicans ATCC 10231 & 13 & & & & \\
\hline
\end{tabular}


Table 4. Cont

\begin{tabular}{|c|c|c|c|c|c|c|}
\hline $\begin{array}{c}\text { Series/ } \\
\text { Compound }\end{array}$ & Strain & $\begin{array}{c}\text { MIC, } \\
\mathrm{mg} \cdot \mathrm{L}^{-1}\end{array}$ & $\begin{array}{c}\mathrm{MBC}, \\
\mathrm{mg} \cdot \mathrm{L}^{-1}\end{array}$ & Method & Notes & Ref. \\
\hline \multirow{10}{*}{91} & E. coli ATCC 25922 & 1.7 & 15 & \multirow{10}{*}{ Broth microdilution } & & \multirow{10}{*}{ [120] } \\
\hline & P. aeruginosa ATCC 6538 & 21 & 8.3 & & & \\
\hline & S. aureus ATCC 278530 & 1.7 & 33 & & & \\
\hline & A. baumannii JCM 6841 & 16 & & & & \\
\hline & B. серасіа JCM 5964 & 64 & & & & \\
\hline & E. hirae ATCC 10541 & 16 & & & & \\
\hline & E. faecalis ATCC 29212 & 19 & & & & \\
\hline & MRSA ATCC 700698 & 8 & & & & \\
\hline & S. epidermidis ATCC 12228 & 9.3 & & & & \\
\hline & C. albicans ATCC 10231 & 27 & & & & \\
\hline \multirow{3}{*}{92} & MRSA ATCC 25923 & $2 \mathrm{ppm}$ & & \multirow{3}{*}{ Broth microdilution } & & \multirow{3}{*}{ [135] } \\
\hline & E. coli ATCC 25922 & $4 \mathrm{pmm}$ & & & & \\
\hline & P. aeruginosa ATCC 27853 & $16 \mathrm{ppm}$ & & & & \\
\hline
\end{tabular}

* MIC, minimum inhibitory concentration; MBC, minimum bactericidal concentration; MRSA, methicillin-resistant S. aureus; only leader compounds from the series are listed in the table.

\subsubsection{Dicationic Ionic Liquids}

A number of dicationic ILs have been tested for their antimicrobial activity (see Figure 30, Table 5, and Table S3 for several examples) [90,136-139]. The high bactericidal activity of some of these ILs (in particular, nitro-substituted imidazolium salts) suggests their possible medical applications (see Table 5).

\subsubsection{Multiple-Charged QACs (Multi-QACs)}

Multi-QACs are salts with three or more charged nitrogen atoms in one molecule [8]. This biocide group is rather underexplored compared to mono- and bis-QACs, probably because of the more complicated synthesis and the lack of low-cost platforms for multicharged QAC structures.

Wuest and Minbiole developed a simple synthetic route for obtaining tris- and tetraQACs on the basis of polyamine platforms 93-97 (Figure 31) [71,72,76,140]. The activity of multi-QACs was significantly higher than that of mono-QACs but was comparable to that of bis-QACs.

Several multi-QACs with aromatic fragments in the structure were also obtained (Figure 32). Forman and colleagues demonstrated that tris-derivatives of crystal violet with one alkyl tail 98 had lower activity than mono-QACs. However, analogs containing ethyl groups at the charged nitrogen instead of methyl groups were more active [107]. Gallagher and colleagues found that tris-QACs with two alkyl tails 99 were more effective against Gram-negative bacteria than tris-QACs with one alkyl tail [141,142]. Tris-pyridinium salts 100 [143] and tetrapyridinium salts 101 [144] also comprised an efficient group of biocides with a broad spectrum of action and surpassed the activity of the well-known pyridinium antiseptic CPC 5 several times. 

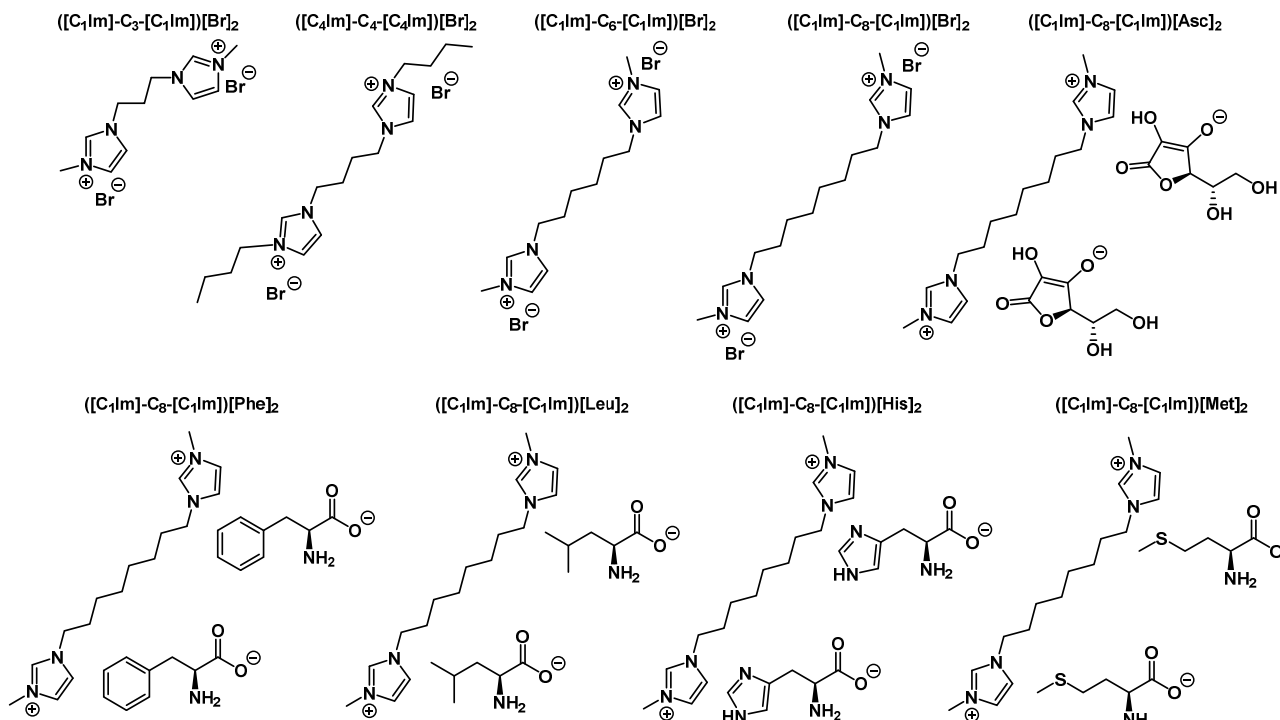

$\left(\left[\mathrm{C}_{1} \mid \mathrm{m}\right]-\mathrm{C}_{8}\left[\mathrm{C}_{1} \mid \mathrm{m}\right]\right)[\mathrm{Leu}]_{2}$

$\left(\left[\mathrm{C}_{1} I \mathrm{~m}\right]-\mathrm{C}_{8}-\left[\mathrm{C}_{1} I \mathrm{~m}\right]\right)[\mathrm{His}]_{2}$

$\left(\left[\mathrm{C}_{1} \mathrm{Im}\right]-\mathrm{C}_{8}-\left[\mathrm{C}_{1} I \mathrm{~m}\right]\right)[\mathrm{Met}]_{2}$

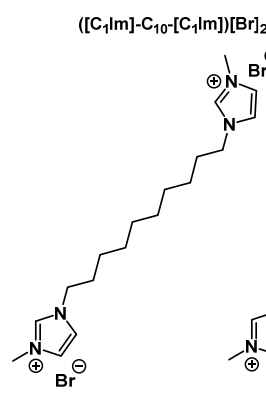

$[\mathrm{Br}]_{2} \quad\left(\left[\mathrm{C}_{1} \mathrm{Im}\right]-\mathrm{C}_{10}-\left[\mathrm{C}_{1} / \mathrm{m}\right]\right)[\mathrm{Asc}]_{2}$<smiles></smiles>

।

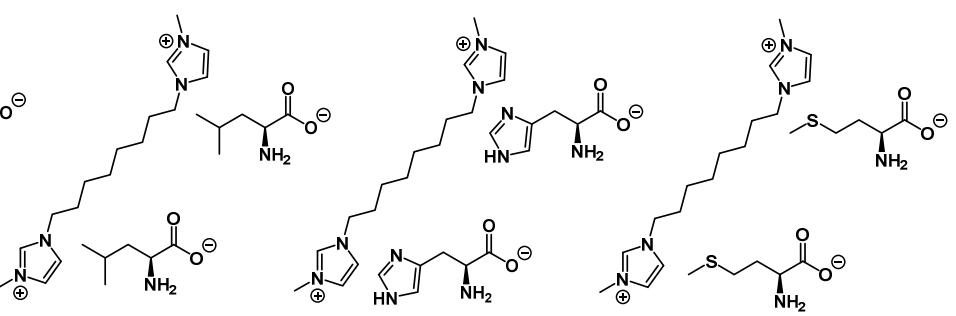

$\left(\left[\mathrm{C}_{1} I \mathrm{~m}\right]-\mathrm{C}_{10}-\left[\mathrm{C}_{1} I \mathrm{~m}\right]\right)[\mathrm{Phe}]_{2}$

([C $\left.\left.\mathrm{C}_{1} I \mathrm{~m}\right]-\mathrm{C}_{10}-\left[\mathrm{C}_{1} \mathrm{Im}\right]\right)[\mathrm{Leu}]_{2}$

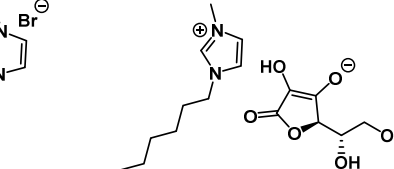

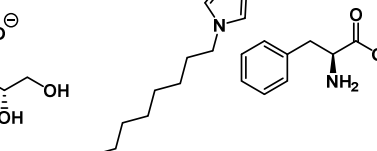

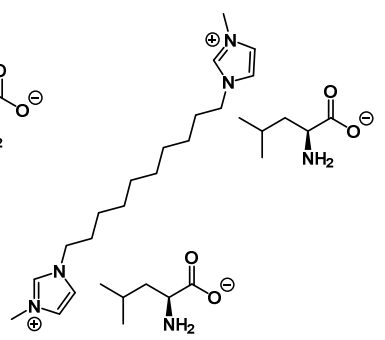

$\left(\left[\mathrm{C}_{1} \mid \mathrm{m}\right]-\mathrm{C}_{10}\left[\mathrm{C}_{1} \mathrm{Im}\right]\right)[\mathrm{His}]_{2}$

([C $\left.\left.\mathrm{C}_{1} I \mathrm{~m}\right]-\mathrm{C}_{10}-\left[\mathrm{C}_{1} I \mathrm{~m}\right]\right)[\mathrm{Met}]_{2}$

$\left(\left[\mathrm{C}_{4} I \mathrm{~m}\right]-\mathrm{C}_{6}-\left[\mathrm{C}_{4} I \mathrm{~m}\right]\right)[\mathrm{Br}]_{2}$
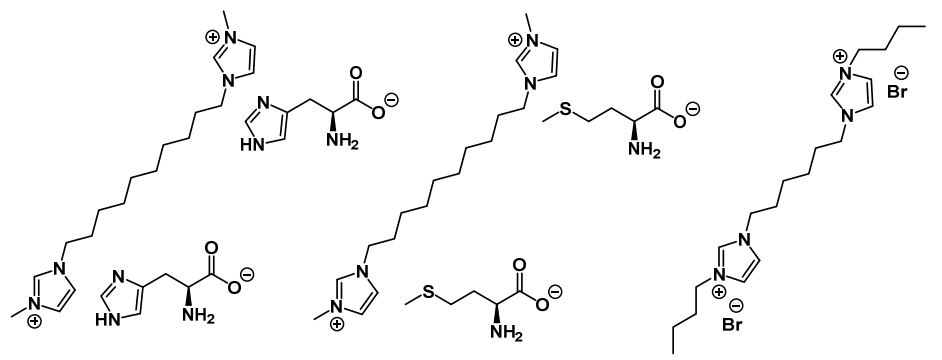

([C ${ }_{1}$ Pyr $\left.]-C_{6}-\left[C_{1} P y r\right]\right)[B r]$
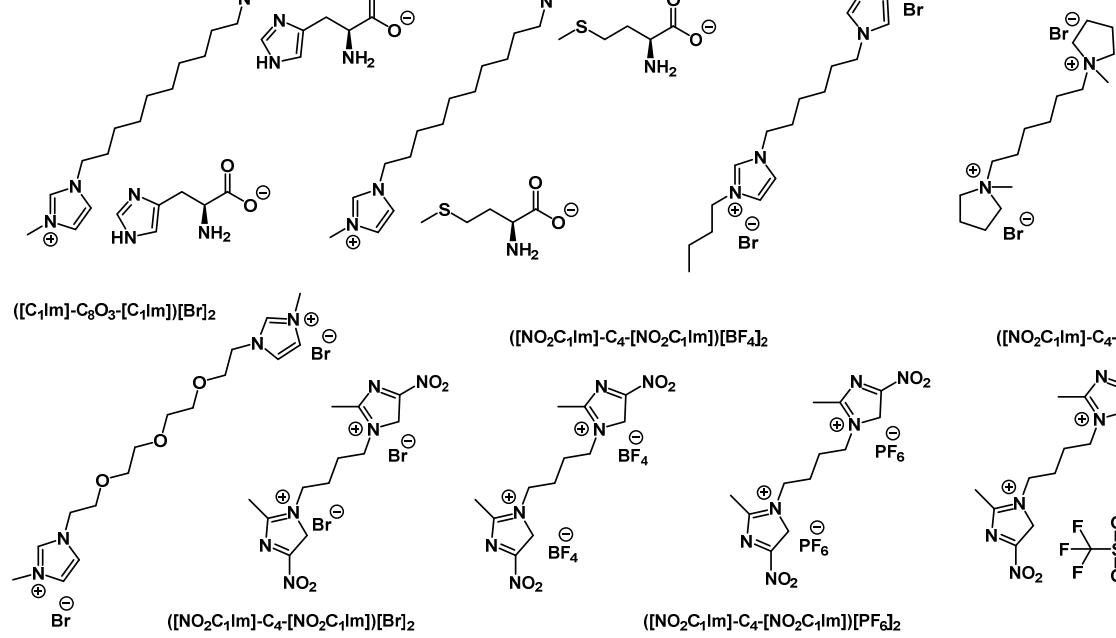

$\left(\left[\mathrm{NO}_{2} \mathrm{C}_{1} I \mathrm{~m}\right]-\mathrm{C}_{4}-\left[\mathrm{NO}_{2} \mathrm{C}_{1} I \mathrm{~m}\right]\right)\left[\mathrm{BF}_{4}\right]_{2}$

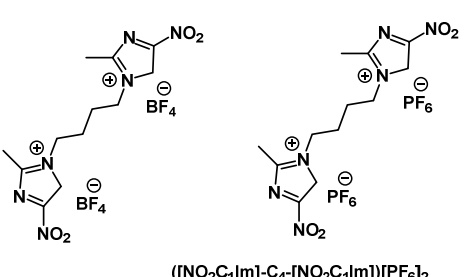

$\left(\left[\mathrm{NO}_{2} \mathrm{C}_{1} \mathrm{Im}\right]-\mathrm{C}_{4}-\left[\mathrm{NO}_{2} \mathrm{C}_{1} \mathrm{Im}\right]\right)[\mathrm{TfO}]_{2}$

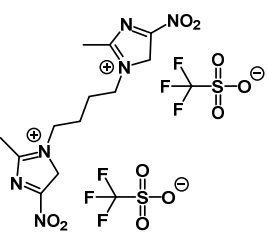

$\left(\left[\mathrm{NO}_{2} \mathrm{C}_{1} \mathrm{Im}\right]-\mathrm{C}_{4}-\left[\mathrm{NO}_{2} \mathrm{C}_{1} \mathrm{Im}\right]\right)\left[\mathrm{PF}_{6}\right]_{2}$

Figure 30. Examples of dicationic ILs with tested antimicrobial activity. The numbers of substances correspond to those in Table 5. 


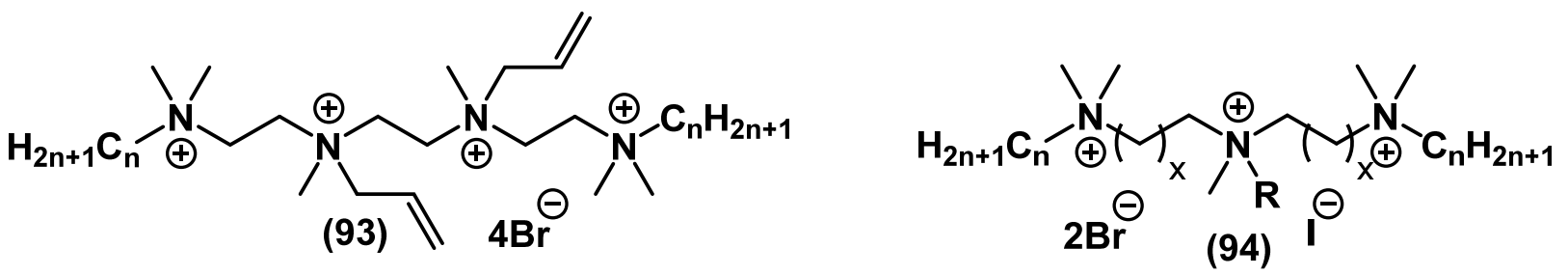

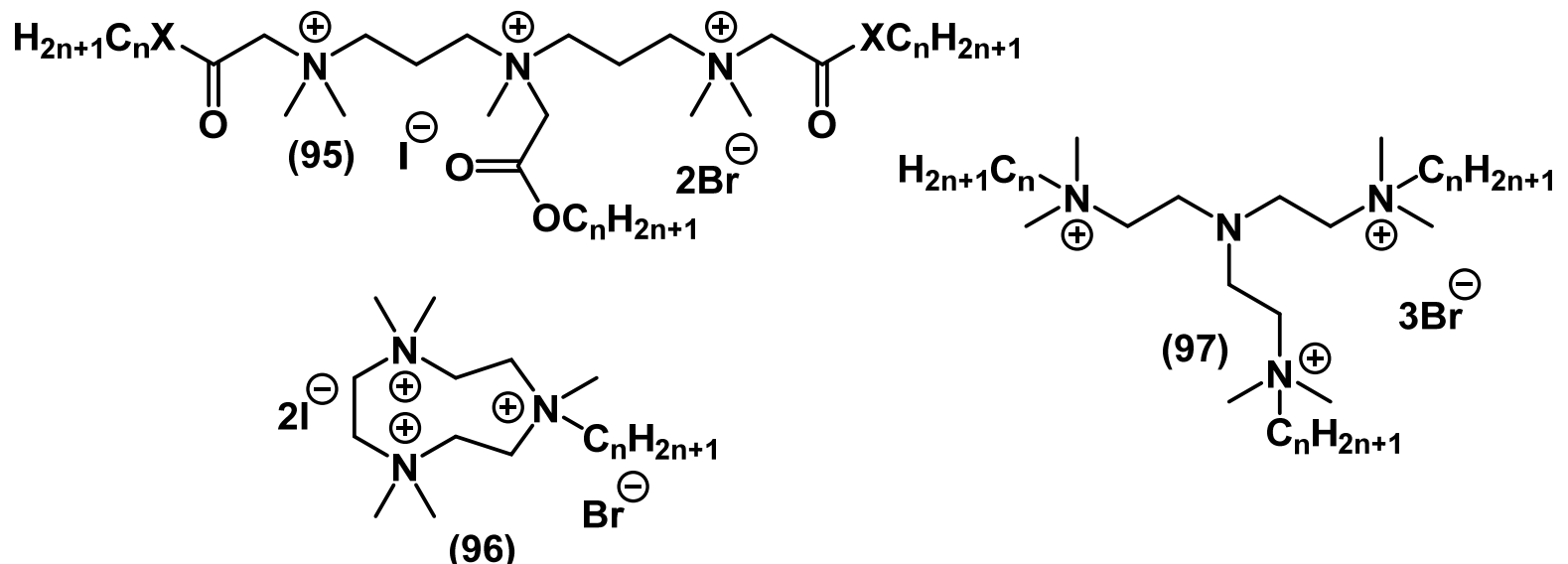

Figure 31. Alkyl multi-QACs.<smiles>CN(C)c1ccc(C(c2ccc([N+](C)(C)C)cc2)c2ccc([N+](C)(C)C)cc2)cc1</smiles><smiles>CCN(C)Cc1cc(C[N+](C)(C)C)cc(C[N+](C)(C)C)c1</smiles>

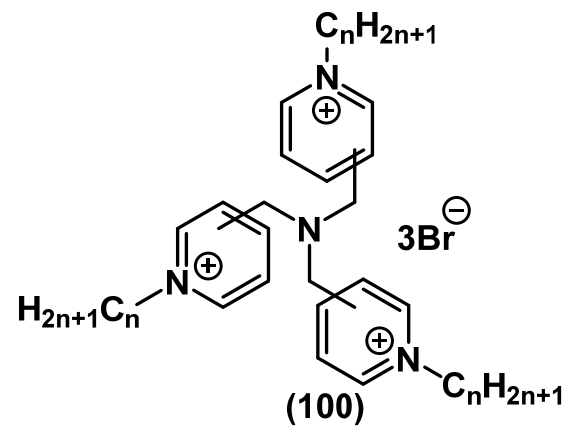

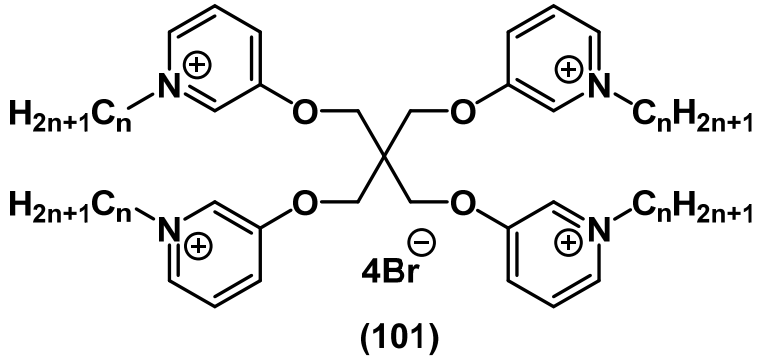

Figure 32. Multi-QACs with aromatic fragments.

An overview of the antibacterial activity of multiple QACs, analyzed in the review, is shown in Table 6. 
Table 5. Antimicrobial activity of dicationic ILs *

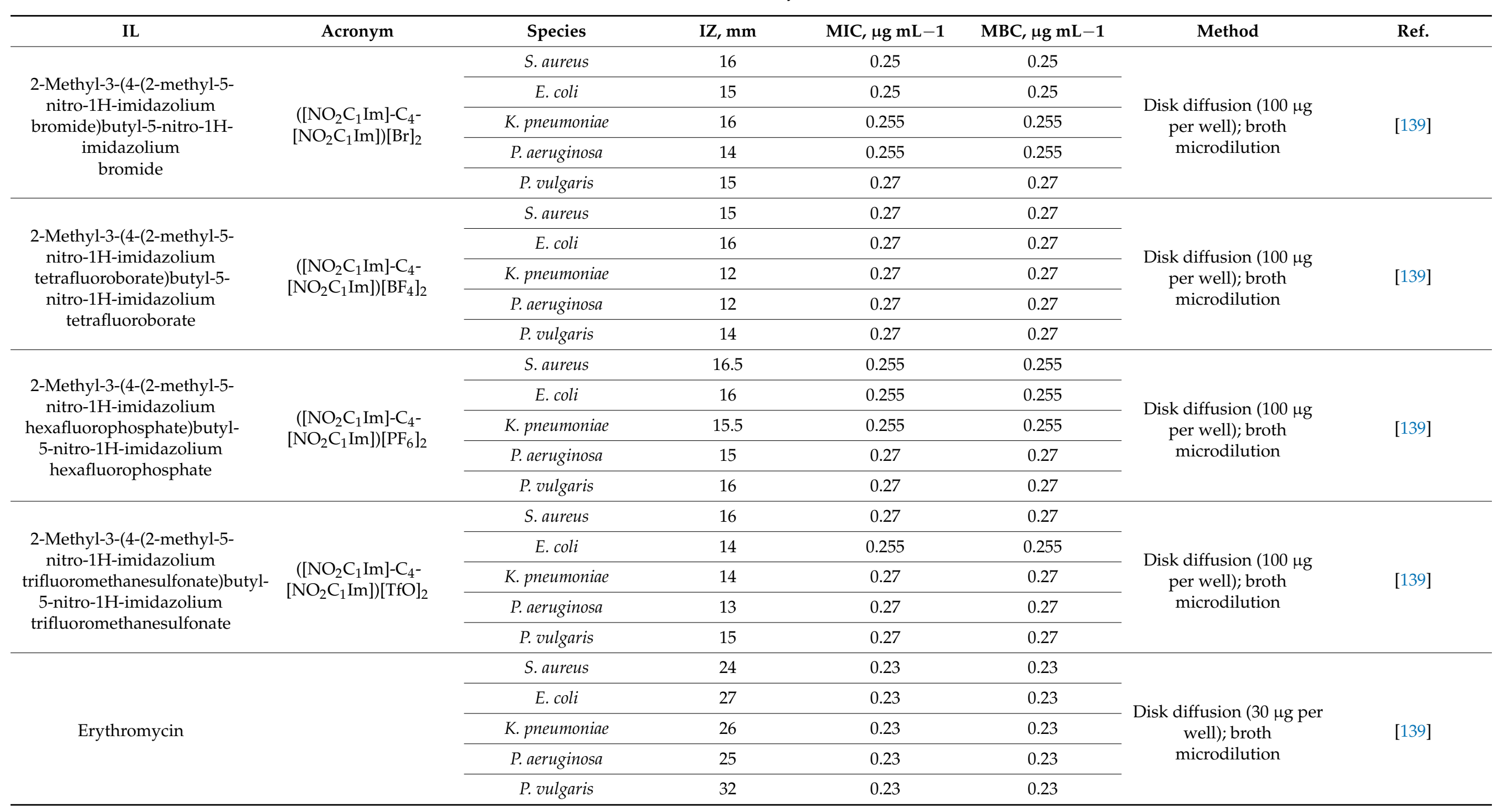


Table 5. Cont.

\begin{tabular}{|c|c|c|c|c|c|c|c|}
\hline IL & Acronym & Species & $\mathrm{IZ}, \mathrm{mm}$ & $\mathrm{MIC}, \mu \mathrm{g} \mathrm{mL}-1$ & $\mathrm{MBC}, \mu \mathrm{g} \mathrm{mL}-1$ & Method & Ref. \\
\hline \multirow{5}{*}{ Nalidixic acid } & & S. aureus & 22 & 0.23 & 0.23 & \multirow{5}{*}{$\begin{array}{l}\text { Disk diffusion }(30 \mu \mathrm{g} \\
\text { per well); broth } \\
\text { microdilution }\end{array}$} & \multirow{5}{*}{ [139] } \\
\hline & & E. coli & 22 & 0.23 & 0.23 & & \\
\hline & & K. pneumoniae & 27 & 0.23 & 0.23 & & \\
\hline & & P. aeruginosa & 21 & 0.23 & 0.23 & & \\
\hline & & P. vulgaris & 24 & 0.23 & 0.23 & & \\
\hline \multirow{5}{*}{ Amikacin } & & S. aureus & 19 & 0.23 & 0.23 & \multirow{5}{*}{$\begin{array}{l}\text { Disk diffusion }(30 \mu \mathrm{g} \\
\text { per well); broth } \\
\text { microdilution }\end{array}$} & \multirow{5}{*}{ [139] } \\
\hline & & E. coli & 20 & 0.23 & 0.23 & & \\
\hline & & K. pneumoniae & 19 & 0.23 & 0.23 & & \\
\hline & & P. aeruginosa & 17 & 0.23 & 0.23 & & \\
\hline & & P. vulgaris & 17 & 0.23 & 0.23 & & \\
\hline
\end{tabular}

* IZ, inhibition zone; MIC, minimum inhibitory concentration; MBC, minimum bactericidal concentration. 
Table 6. Antimicrobial activity of multi-QACs *.

\begin{tabular}{|c|c|c|c|c|c|}
\hline $\begin{array}{c}\text { Series/ } \\
\text { Compound }\end{array}$ & Strain & $\mathrm{MIC}, \mathrm{mg} \cdot \mathrm{L}^{-1}$ & Method & Notes & Ref. \\
\hline \multirow{4}{*}{93} & S. aureus SH1000 & $1 \mu \mathrm{M}$ & \multirow{4}{*}{ Broth microdilution } & & \multirow{4}{*}{ [71] } \\
\hline & E. faecalis OG1RF & $1 \mu \mathrm{M}$ & & & \\
\hline & E. coli MC4100 & $1 \mu \mathrm{M}$ & & & \\
\hline & P. aeruginosa PAO1-WT & $2 \mu \mathrm{M}$ & & & \\
\hline \multirow{4}{*}{94} & S. aureus SH1000 & $0.5 \mu \mathrm{M}$ & \multirow{4}{*}{ Broth microdilution } & & \multirow{4}{*}{ [71] } \\
\hline & E. faecalis OG1RF & $1 \mu \mathrm{M}$ & & & \\
\hline & E. coli MC4100 & $1 \mu \mathrm{M}$ & & & \\
\hline & P. aeruginosa PAO1-WT & $4 \mu \mathrm{M}$ & & & \\
\hline \multirow{3}{*}{95} & S. aureus SH1000 & $1 \mu \mathrm{M}$ & \multirow{3}{*}{ Broth microdilution } & & \multirow{3}{*}{ [112] } \\
\hline & MRSA 300-0114 & $0.5 \mu \mathrm{M}$ & & & \\
\hline & MRSA ATCC 33592 & $1 \mu \mathrm{M}$ & & & \\
\hline \multirow{4}{*}{96} & S. aureus SH1000 & $1 \mu \mathrm{M}$ & \multirow{4}{*}{ Broth microdilution } & & \multirow{4}{*}{ [72] } \\
\hline & MRSA 300-0114 & $1 \mu \mathrm{M}$ & & & \\
\hline & E. coli MC4100 & $2 \mu \mathrm{M}$ & & & \\
\hline & P. aeruginosa PAO1-WT & $4 \mu \mathrm{M}$ & & & \\
\hline \multirow{6}{*}{96} & S. aureus SH1000 & $0.5 \mu \mathrm{M}$ & \multirow{6}{*}{ Broth microdilution } & & \multirow{6}{*}{ [140] } \\
\hline & MRSA 300-0114 & $0.5 \mu \mathrm{M}$ & & & \\
\hline & MRSA ATCC 33592 & $0.5 \mu \mathrm{M}$ & & & \\
\hline & E. faecalis OG1RF & $1 \mu \mathrm{M}$ & & & \\
\hline & E. coli MC4100 & $0.5 \mu \mathrm{M}$ & & & \\
\hline & P. aeruginosa PAO1-WT & $0.5 \mu \mathrm{M}$ & & & \\
\hline \multirow{6}{*}{98} & S. aureus SH1000 & $1 \mu \mathrm{M}$ & \multirow{6}{*}{ Broth microdilution } & & \multirow{6}{*}{ [107] } \\
\hline & MRSA 300-0114 & $0.5 \mu \mathrm{M}$ & & & \\
\hline & MRSA ATCC 33592 & $0.5 \mu \mathrm{M}$ & & & \\
\hline & E. faecalis OG1RF & $1 \mu \mathrm{M}$ & & & \\
\hline & E. coli MC4100 & $0.5 \mu \mathrm{M}$ & & & \\
\hline & P. aeruginosa PAO1-WT & $4 \mu \mathrm{M}$ & & & \\
\hline \multirow{6}{*}{99} & B. cereus & $2 \mu \mathrm{M}$ & \multirow{6}{*}{ Broth microdilution } & & \multirow{6}{*}{ [141] } \\
\hline & E. faecalis ATCC 29212 & $2 \mu \mathrm{M}$ & & & \\
\hline & S. agalactiae $\mathrm{J} 48$ & $2 \mu \mathrm{M}$ & & & \\
\hline & S. aureus ATCC 29213 & $2 \mu \mathrm{M}$ & & & \\
\hline & E. coli ATCC 25922 & $4 \mu \mathrm{M}$ & & & \\
\hline & P. aeruginosa ATCC 27853 & $16 \mu \mathrm{M}$ & & & \\
\hline \multirow{6}{*}{100} & S. aureus SH1000 & $0.5 \mu \mathrm{M}$ & \multirow{6}{*}{ Broth microdilution } & & \multirow{6}{*}{ [143] } \\
\hline & E. faecalis OG1RF & $1 \mu \mathrm{M}$ & & & \\
\hline & E. coli MC4100 & $1 \mu \mathrm{M}$ & & & \\
\hline & P. aeruginosa PAO1-WT & $2 \mu \mathrm{M}$ & & & \\
\hline & MRSA 300-0114 & $0.5 \mu \mathrm{M}$ & & & \\
\hline & MRSA ATCC 33592 & $0.5 \mu \mathrm{M}$ & & & \\
\hline \multirow{3}{*}{101} & MRSA ATCC 25923 & 4 & & & \\
\hline & E. coli ATCC 25922 & 4 & Broth microdilution & tetra-pyridinic & [144] \\
\hline & P. aeruginosa ATCC 27853 & 32 & & & \\
\hline
\end{tabular}

${ }^{*} \mathrm{MIC}$, minimum inhibitory concentration; MBC, minimum bactericidal concentration; MRSA, methicillin-resistant $S$. aureus; only leader compounds from the series are listed in the table. 


\subsubsection{Poly-Charged QACs (Poly-QACs)}

Polymer structures with quaternary nitrogen occupy a large niche in the field of cationic biocides. QACs exhibiting antimicrobial activity can be incorporated into polymer structures in several ways [49]:

Ring-opening polymerization. Chain-growth polymerization, in which one end of the polymer chain carries an active site for adding cyclic monomers. The terminal groups of the resulting polymer depend on the initiator used and the termination reaction [145].

Controlled radical polymerization. Continuous polymerization includes several stages: Initiation, growth, and chain termination [146].

Click reaction. Polymerization that utilizes methods of click chemistry [147].

Similar to other types of QACs, the structure of poly-QACs can vary depending on the monomer composition (homogeneous poly-QACs (Figure 33) in the case of the same monomers, or copolymers (Figure 34 ) in the case of different monomers) and the polymerization type.

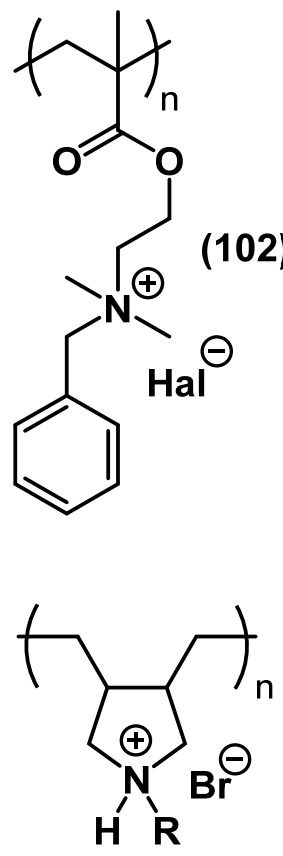<smiles>CC(C)(C)CC(CC(C)(C)C)c1ccc(C[N+](C)(C)Cl)cc1</smiles><smiles>CCCCCCCC[N+](C)(C)Cc1ccc(C[N+](C)(C)C)cc1</smiles>

(104)

(105)

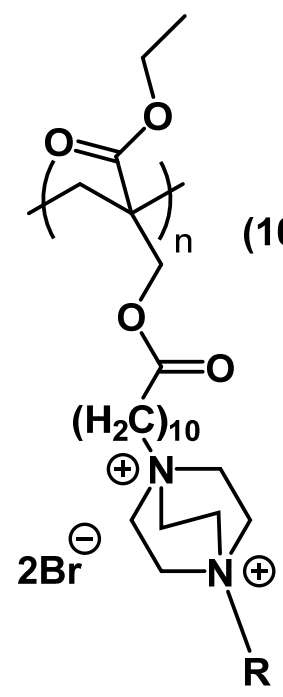

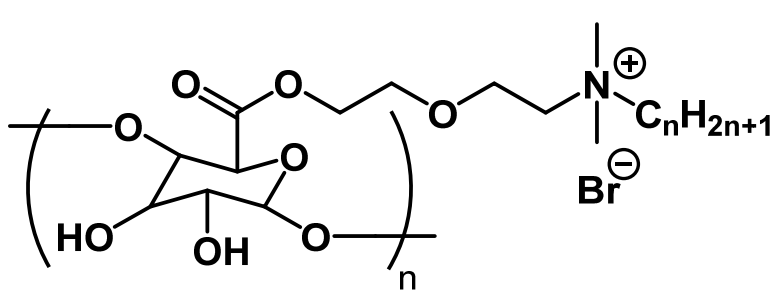

(107)

(106)

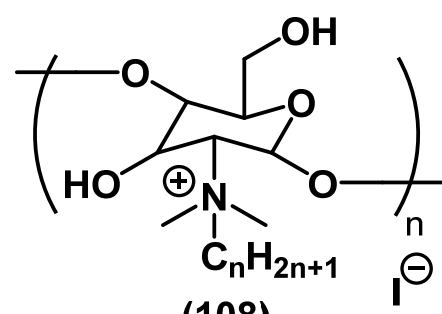

(108)

Figure 33. Spectrum of biologically active homogeneous poly-QACs.

Lu and colleagues studied the biological properties of poly-QACs with benzyl substituents and ether groups in side chain 102 [148]. The activity of the polyderivatives was significantly higher than that of the corresponding monomers; it increased upon increasing the length of the alkyl substituent. Guo and colleagues compared polymers with quaternary nitrogen in the side $\mathbf{1 0 3}$ and main $\mathbf{1 0 4}$ chains [149]. The presence of charged nitrogen atoms in the main polymer chain enhanced the antibacterial effect on Gram-positive and Gram-negative bacteria by several times. The carbohydrate-based poly-QACs obtained by 
Badawy's 108 [150] and Shaban's 107 [151] groups also exhibited biocidal activity. Polymer salts consisting of monomers with DABCO-containing heterocyclic QACs 106 were obtained by Mathias' group [152]. Researchers observed an increase in bactericidal activity with the growth of alkyl chains. It should be noted that the monomer did not exhibit antibacterial activity. Polymerization may be the key to achieving the required biocidal effect for inactive QAC molecules. Timofeeva and colleagues developed an approach to the synthesis of quaternary poly(diallyldialkylammonium) salts with various substituents 105 [153]. The researchers noted that the antibacterial effect, but not the antifungal effect, became more pronounced upon increasing the mass of the polymer.

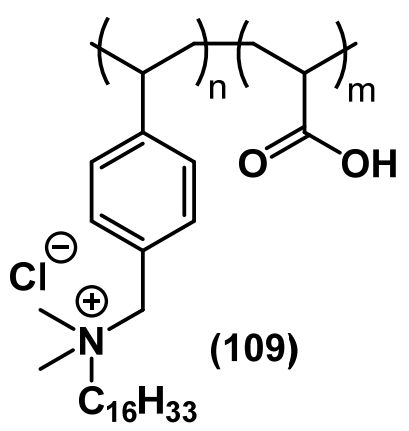<smiles>CCCCCCC(C)C(C(C(=O)OCC1CO1)C(C)(C)C)C(C)(C)C</smiles><smiles>CCCCCCC(C)C(C)C(C)C(C)C(C)c1ccc(S(=O)(=O)N(C)C)cc1</smiles><smiles>CC(C)C(C(=O)OCCN)C(=O)OCC[N+](C)(C)C</smiles><smiles>CC(C)C(C)c1ccc(CN2C(=O)N(Cl)C(C)(C)C2=O)cc1CN(C)[N+](C)(C)Cl</smiles>

Figure 34. Copolymer poly-QACs.

Kallitsis and colleagues studied single- 109-110 and two-charged 111 copolymeric QACs in their work $[154,155]$. The peculiarity of this study was in the fact that the polymer chain in one of the target compounds $\mathbf{1 1 0}$ was an anion, while the cation was a conventional mono-QAC alkyl cation of CTAB type 2, whereas compound $\mathbf{1 1 1}$ was poly-QAC bearing both cations and anions. This composition had a positive impact on the biocidal effect against a wide range of bacteria. The optimal structure was established as $75 \%$ ionic and $25 \%$ covalent bonds of the polymer with QAC. Jie and colleagues combined the QAC and $\mathrm{N}$-chloramine 113 molecules in one polymer [128]. A similar successful approach was pursued by Liu and colleagues [56-58]. Bai and colleagues synthesized a polymer combining amino and QAC groups 112, which showed excellent bacteriostatic potential [156].

The diversity of homogeneous and copolymeric QACs is very high and is beyond the scope of this review; only exemplary biologically active representatives of this class are presented here. More detailed information on poly-QACs can be found in other reviews [44,47,49,50,157-159].

An overview of the antibacterial activity of poly-QACs, analyzed in the review, is shown in Table 7. 
Table 7. Antimicrobial activity of poly-QACs *.

\begin{tabular}{|c|c|c|c|c|c|c|}
\hline $\begin{array}{c}\text { Series/ } \\
\text { Compound }\end{array}$ & Strain & $\mathrm{MIC}, \mathrm{mg} \cdot \mathrm{L}^{-1}$ & $\mathrm{MBC}, \mathrm{mg} \cdot \mathrm{L}^{-1}$ & Method & Notes & Ref. \\
\hline \multirow{2}{*}{102} & E. coli ATCC 25922 & & 1.56 & \multirow{2}{*}{$\begin{array}{l}\text { Broth } \\
\text { microdilution }\end{array}$} & & \multirow{2}{*}{ [148] } \\
\hline & S. aureus ATCC 25923 & & 1.56 & & & \\
\hline \multirow{2}{*}{103} & E. coli ATCC 8099 & 0.78 & & \multirow{2}{*}{$\begin{array}{l}\text { Broth } \\
\text { microdilution }\end{array}$} & & \multirow{2}{*}{ [149] } \\
\hline & S. aureus ATCC 6538 & 0.91 & & & & \\
\hline \multirow{2}{*}{104} & E. coli ATCC 8099 & 0.13 & & \multirow{2}{*}{$\begin{array}{l}\text { Broth } \\
\text { microdilution }\end{array}$} & & \multirow{2}{*}{ [149] } \\
\hline & S. aureus ATCC 6538 & 0.28 & & & & \\
\hline \multirow{6}{*}{105} & E. coli ATCC 25922 & 7 & & \multirow{6}{*}{$\begin{array}{l}\text { Broth tube } \\
\text { dilution }\end{array}$} & & \multirow{6}{*}[153]{} \\
\hline & S. aureus ATCC $6538 \mathrm{P}$ & 7 & & & & \\
\hline & $\begin{array}{c}\text { C. albicans } \\
\text { ATCC 865-653 }\end{array}$ & 3.5 & & & & \\
\hline & $\begin{array}{l}\text { P. aeruginosa } \\
\text { ATCC } 9027\end{array}$ & 31 & & & & \\
\hline & P. mirabilis 47 & 31 & & & & \\
\hline & $\begin{array}{l}\text { K. pneumoniae } \\
\text { ATCC } 13883\end{array}$ & 62 & & & & \\
\hline \multirow{2}{*}{106} & E. coli & 62.5 & 62.5 & \multirow{2}{*}{ Broth dilution } & & \multirow{2}{*}{ [152] } \\
\hline & S. aureus & 62.5 & 62.5 & & & \\
\hline \multirow{5}{*}{107} & E. coli & $22 \mathrm{~mm} / \mathrm{mg}(\mathrm{IZ})$ & & \multirow{5}{*}{ Disk diffusion } & \multirow{5}{*}{$\begin{array}{c}\text { Possesses } \\
\text { anticorrosion } \\
\text { activity }\end{array}$} & \multirow{5}{*}[151]{} \\
\hline & S. aureus & $20 \mathrm{~mm} / \mathrm{mg}(\mathrm{IZ})$ & & & & \\
\hline & C. albicans & $13 \mathrm{~mm} / \mathrm{mg}(\mathrm{IZ})$ & & & & \\
\hline & P. aeruginosa & $24 \mathrm{~mm} / \mathrm{mg}(\mathrm{IZ})$ & & & & \\
\hline & A. niger & $12 \mathrm{~mm} / \mathrm{mg}(\mathrm{IZ})$ & & & & \\
\hline \multirow{3}{*}{108} & B. cinerea & 106 & & \multirow{3}{*}{$\begin{array}{l}\text { Radial growth } \\
\text { technique }\end{array}$} & \multirow{3}{*}{$\begin{array}{l}\text { Efficient against } \\
\text { fungal spores }\end{array}$} & \multirow{3}{*}[150]{} \\
\hline & F. oxysporum & 720 & & & & \\
\hline & P. debaryanum & 164 & & & & \\
\hline \multirow{2}{*}{109} & S. aureus & $\begin{array}{l}5.3(\log \\
\text { reduction, } 24 \mathrm{~h} \\
\text { contact) }\end{array}$ & & \multirow{2}{*}{ Plate count } & \multirow{4}{*}{$\begin{array}{l}\text { Prevent } \\
\text { biofouling }\end{array}$} & \multirow{2}{*}[155]{} \\
\hline & P. aeruginosa & $\begin{array}{l}5.4(\log \\
\text { reduction, } 24 \mathrm{~h} \\
\text { contact) }\end{array}$ & & & & \\
\hline \multirow{2}{*}{110} & S. aureus & $\begin{array}{l}1.7(\log \\
\text { reduction, } 24 \mathrm{~h} \\
\text { contact) }\end{array}$ & & \multirow{2}{*}{ Plate count } & & [155] \\
\hline & P. aeruginosa & $\begin{array}{l}1.9(\log \\
\text { reduction, } 24 \mathrm{~h} \\
\text { contact) }\end{array}$ & & & & {$[100]$} \\
\hline & S. aureus & $\begin{array}{l}6 \text { (log reduction, } \\
24 \mathrm{~h} \text { contact) }\end{array}$ & & & & \\
\hline 111 & E. coli & $\begin{array}{l}6 \text { (log reduction, } \\
24 \mathrm{~h} \text { contact) }\end{array}$ & & Plate count & & [154] \\
\hline & P. aeruginosa & $\begin{array}{c}4.5(\log \\
\text { reduction, } 24 \mathrm{~h} \\
\text { contact) }\end{array}$ & & & & \\
\hline 112 & S. aureus & 128 & & Ploto count & & [156] \\
\hline 112 & E. coli & 256 & & Plate count & & {$[100]$} \\
\hline & S. aureus ATCC 6538P & $\begin{array}{c}7.26(\log \\
\text { reduction, } 1 \mathrm{~min} \\
\text { contact })\end{array}$ & & & & \\
\hline 113 & E. coli ATCC 1122 & $\begin{array}{l}8.26(\log \\
\text { reduction, } 1 \mathrm{~min} \\
\text { contact })\end{array}$ & & Plate count & & [160] \\
\hline
\end{tabular}

* IZ, inhibition zone; MIC, minimum inhibitory concentration; MBC, minimum bactericidal concentration; MRSA, methicillin-resistant S. aureus; only leader compounds from the series are listed in the table. 


\subsubsection{Polyionic Liquids}

According to the strict definition, poly-ILs are ionic polymers with complete ionicity [161]. However, ionic polymers with lower levels of ionicity are often considered poly-ILs in publications. In recent years, poly-ILs have been extensively studied as advantageous materials for antibacterial coatings and surfaces [89,162-169]. Exemplary poly-ILs with tested antibacterial activity are listed in Table 8 and Figure 36. Note that the table includes substances 103 and 104, which are also considered poly-(QACs).
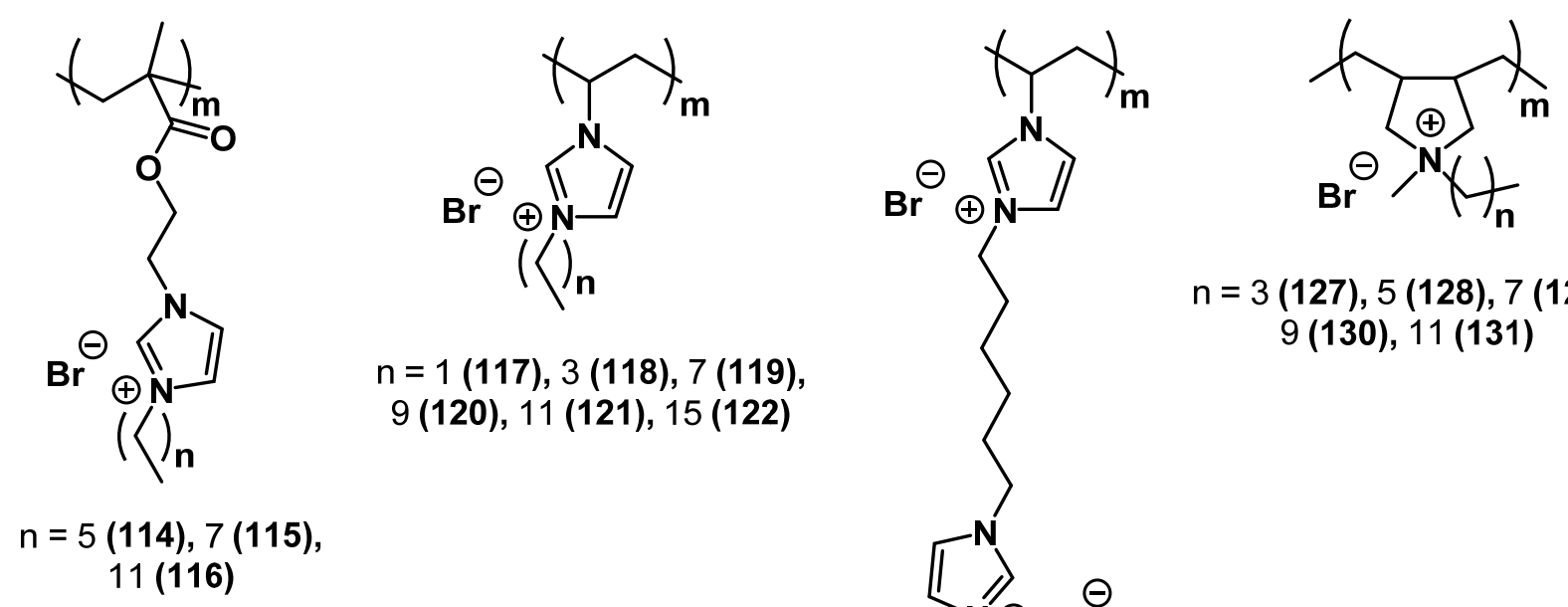

$$
\begin{gathered}
n=3(127), 5(128), 7(129), \\
9(130), 11(131)
\end{gathered}
$$
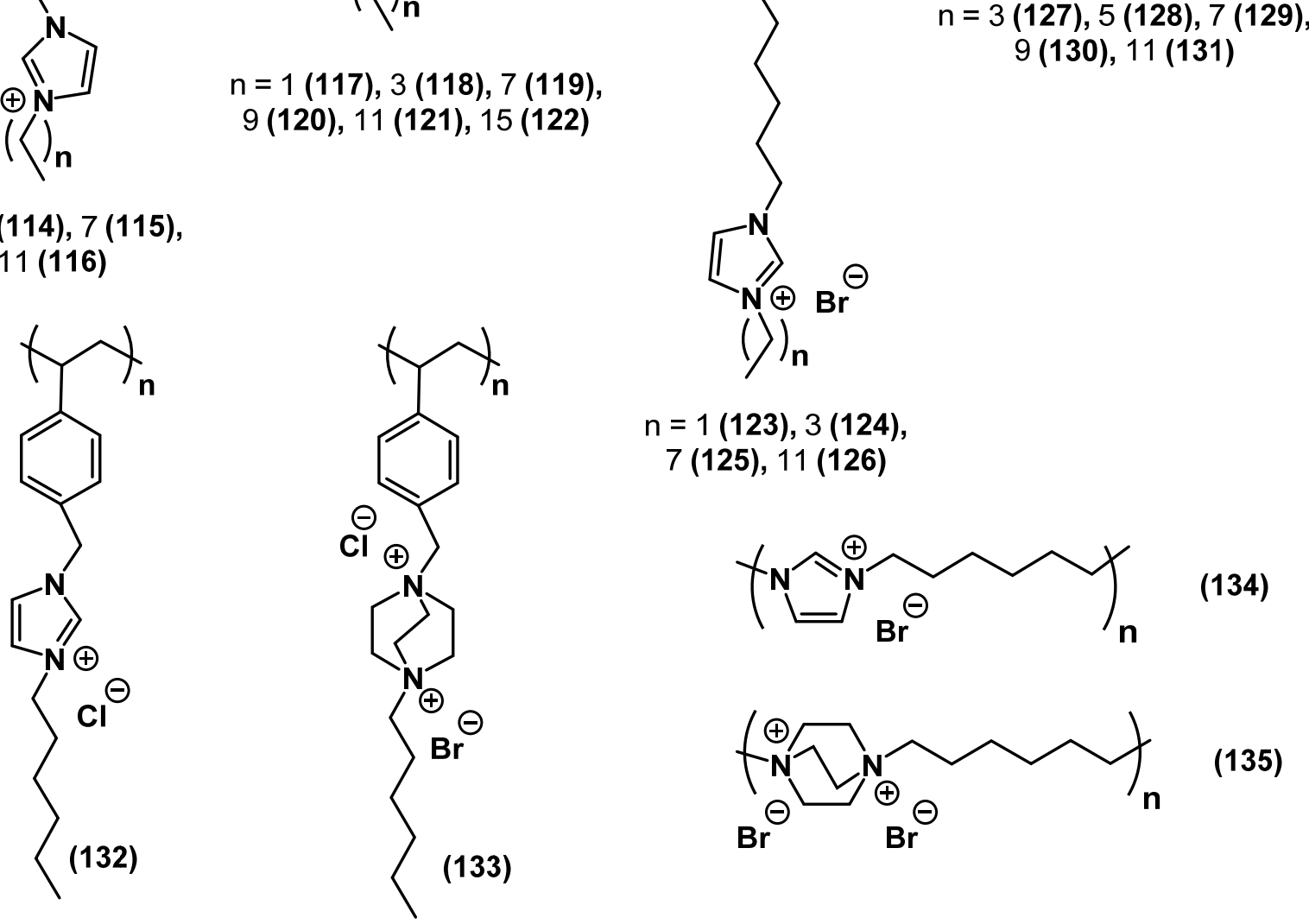

Figure 35. Examples of poly-ILs with tested antimicrobial activity. The numbers of substances correspond to those in Table 8. 
Table 8. Antimicrobial activity of poly-ILs *.

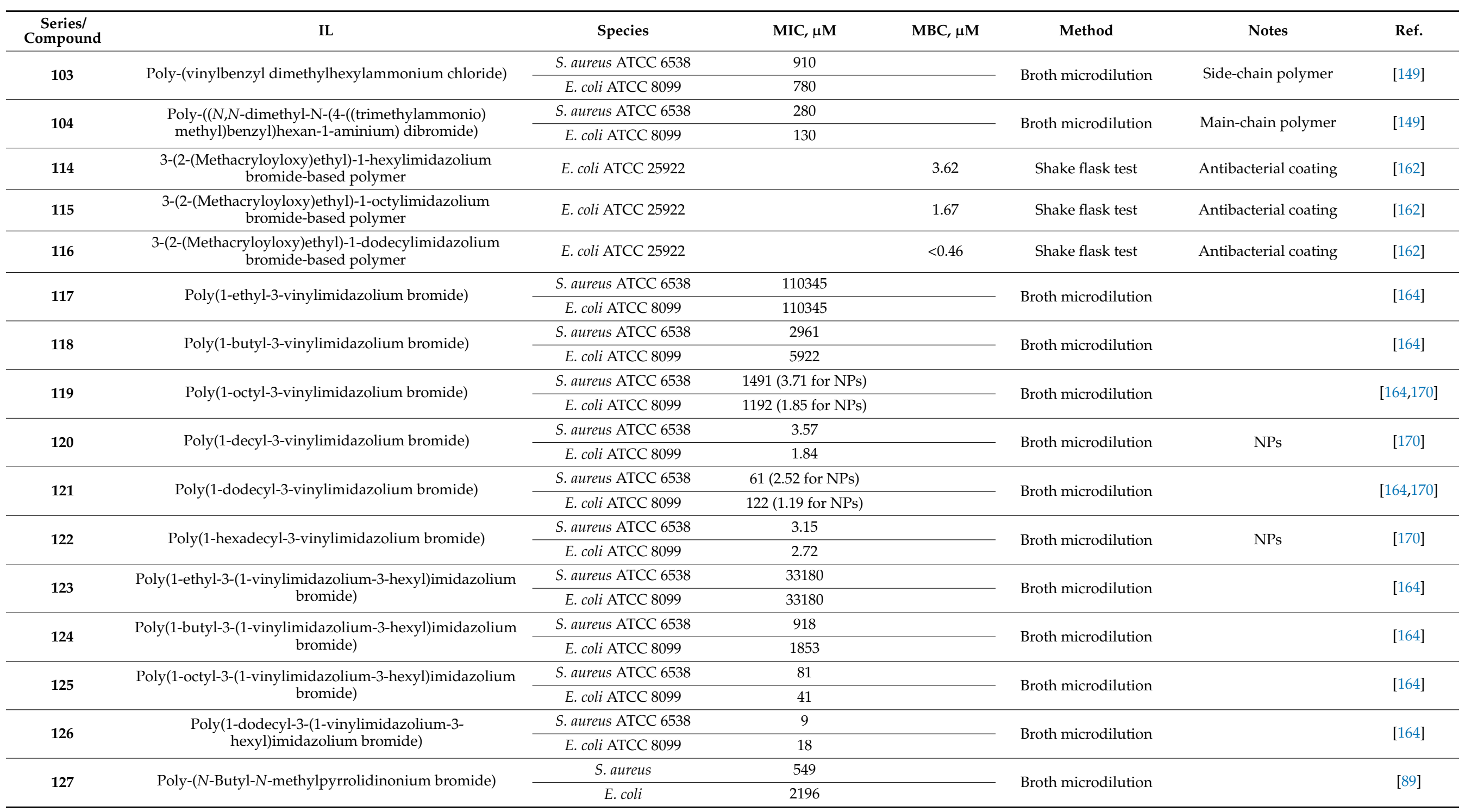


Table 8. Cont.

\begin{tabular}{|c|c|c|c|c|c|c|c|}
\hline $\begin{array}{c}\text { Series/ } \\
\text { Compound }\end{array}$ & IL & Species & $\mathrm{MIC}, \mu \mathrm{M}$ & MBC, $\mu \mathrm{M}$ & Method & Notes & Ref. \\
\hline \multirow{2}{*}{128} & \multirow{2}{*}{ Poly-(N-Hexyl- $N$-methylpyrrolidinonium bromide) } & S. aureus & 236 & & \multirow{2}{*}{ Broth microdilution } & & \multirow{2}{*}{ [89] } \\
\hline & & E. coli & 548 & & & & \\
\hline \multirow{2}{*}{129} & \multirow{2}{*}{ Poly-(N-Octyl- $N$-methylpyrrolidinonium bromide) } & S. aureus & 147 & & \multirow{2}{*}{ Broth microdilution } & & \multirow{2}{*}{ [89] } \\
\hline & & E. coli & 424 & & & & \\
\hline \multirow{2}{*}{130} & \multirow{2}{*}{ Poly-(N-Decyl-N-methylpyrrolidinonium bromide) } & S. aureus & 112 & & \multirow{2}{*}{ Broth microdilution } & & \multirow{2}{*}{ [89] } \\
\hline & & E. coli & 224 & & & & \\
\hline \multirow{2}{*}{131} & \multirow{2}{*}{ Poly-(N-Dodecyl- $N$-methylpyrrolidinonium bromide) } & S. aureus & 61 & & \multirow{2}{*}{ Broth microdilution } & & \multirow{2}{*}{ [89] } \\
\hline & & E. coli & 90 & & & & \\
\hline \multirow{2}{*}{132} & \multirow{2}{*}{ Poly-(1-vinylbenzyl-3-hexylimidazolium chloride) } & S. aureus ATCC 6538 & 900 & & \multirow{2}{*}{ Broth microdilution } & \multirow{2}{*}{ Side-chain polymer } & \multirow{2}{*}{ [149] } \\
\hline & & E. coli ATCC 8099 & 770 & & & & \\
\hline \multirow{2}{*}{133} & \multirow{2}{*}{$\begin{array}{l}\text { Poly-(1-vinylbenzyl-4-hexyl-1,4-diazoniabicyclo[2 } \\
\text {.2.2]octane-1,4-diium chloride bromide) }\end{array}$} & S. aureus ATCC 6538 & 1280 & & \multirow{2}{*}{ Broth microdilution } & \multirow{2}{*}{ Side-chain polymer } & \multirow{2}{*}{ [149] } \\
\hline & & E. coli ATCC 8099 & 1160 & & & & \\
\hline \multirow{2}{*}{134} & \multirow{2}{*}{ Poly-(1-hexyl-3-methylimidazolium bromide) } & S. aureus ATCC 6538 & 230 & & \multirow{2}{*}{ Broth microdilution } & \multirow{2}{*}{ Main-chain polymer } & \multirow{2}{*}{ [149] } \\
\hline & & E. coli ATCC 8099 & 110 & & & & \\
\hline 135 & $\begin{array}{l}\text { Poly-(1-hexyl-4-methyl-1,4-diazoniabicyclo[2.2.2]octane- } \\
\text { 1,4-diium dibromide) }\end{array}$ & S. aureus ATCC 6538 & 560 & & Broth microdilution & Main-chain polymer & {$[149$} \\
\hline
\end{tabular}

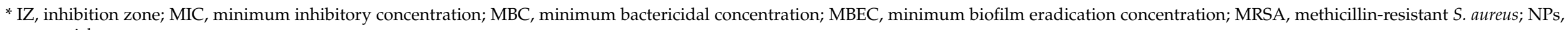
nanoparticles. 

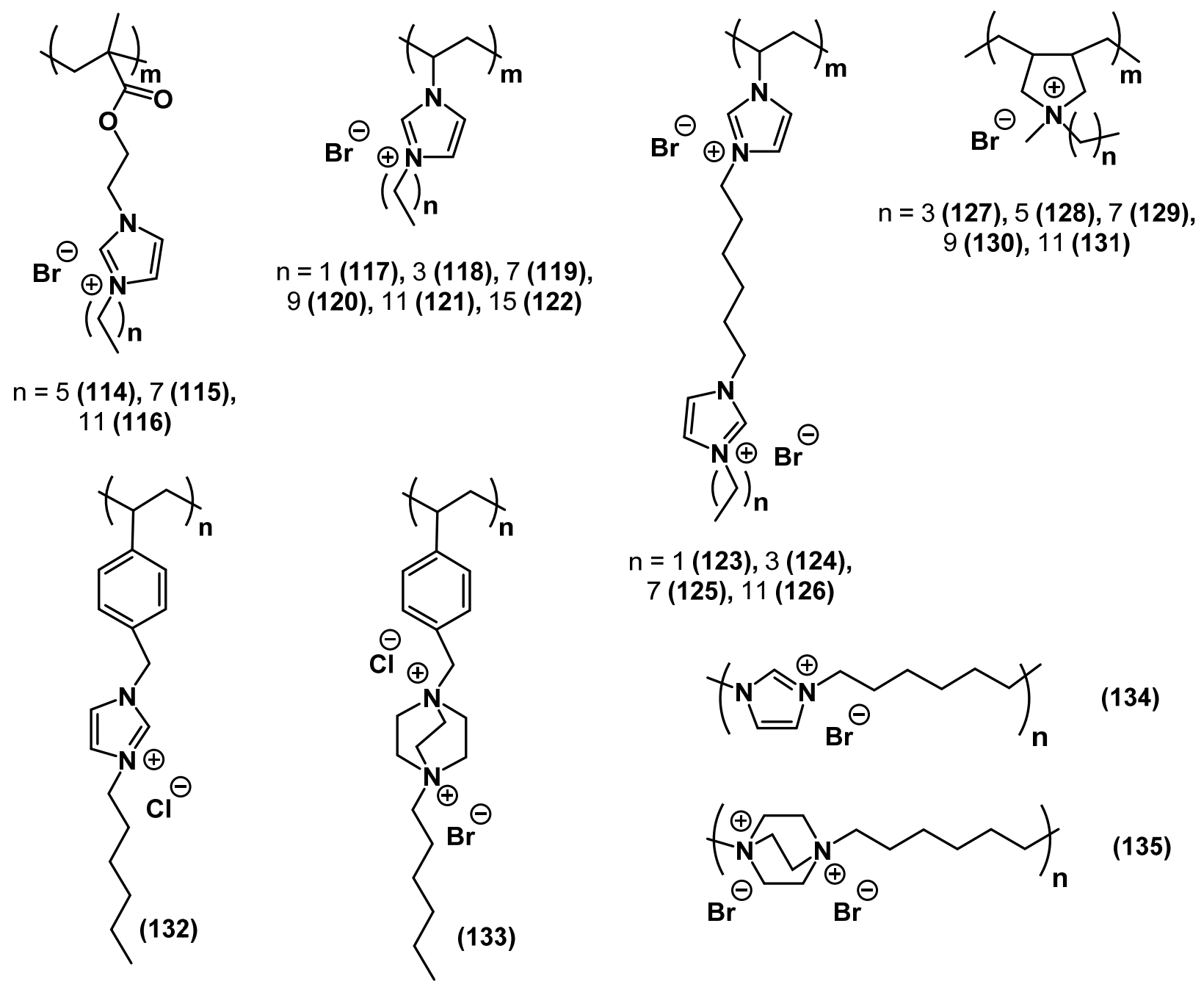

(134)

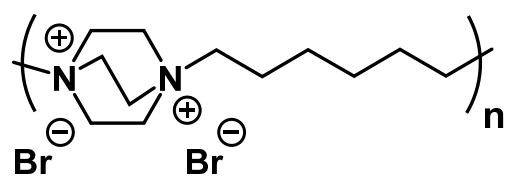

Figure 36. Examples of poly-ILs with tested antimicrobial activity. The numbers of substances correspond to those in Table 8.

Antibacterial coatings on the basis of 3-(2-(methacryloyloxy)ethyl)-1-alkylimidazolium ILs showed high bactericidal activity against E. coli (see entries 114-116 in Table 8) [162]. In the case of 1-alkyl-3-vinylimidazolium-based poly-ILs, the alkyl side chain length and charge density were directly related to the antimicrobial activity against E. coli and S. aureus (see entries 117-119, 121, and 123-126 in Table 8) [164]. In contrast, the bactericidal activity of the corresponding poly-IL membranes increased upon increasing the charge density but decreased upon increasing the alkyl chain length. A similar picture was observed for pyrrolidinium-based ILs and membranes [89]. The homopolymeric ILs were active against S. aureus and E. coli, and their antimicrobial activity increased upon increasing the alkyl side chain length in the monomer (see entries 123-126 and 127-131 in Table 8). The opposite was observed for the corresponding poly-IL-based membranes, which also demonstrated good hemocompatibility and low cytotoxicity. Of note, nanoparticles on the basis of 1-alkyl-3-vinylimidazolium poly-ILs showed significantly higher antimicrobial activity than the original poly-ILs [170] (see entries 119-122 in Table 8).

(2-Ethylhexyl)ethylenediaminium bis(trifluoromethanesulfonyl)imide-loaded ionogel surface coatings efficiently inhibited the growth of various microorganisms, including those from the ESKAPE list, and prevented the formation of biofilms [163]. Microneedle patches on the basis of salicylic acid-containing API-poly-IL were successfully tested 
in the treatment of Propionobacterium acnes skin infections [165]. Ionic graft copolymers on the basis of [2-(methacryloyloxy)ethyl]trimethylammonium chloride were studied as possible delivery systems for ionic drugs ( $p$-aminosalicylate and clavunate) [171]. IL-grafted wound dressings on the basis of 1-vinyl-3-methylimidazolium bromide demonstrated good antimicrobial activity and low cytotoxicity [172,173].

\subsubsection{QAC-Containing Bactericidal Coatings}

QACs also find application in the composition of bioactive materials and antibacterial coatings. This topic is more relevant than ever due to the growing part of the paint and coatings industry in the biocide market. Thus, research on the application of QACs at surfaces continues to expand.

Antimicrobial films based on surface-modified microfibrillated cellulose grafted with mono-QACs showed high antibacterial activity against S. aureus and E. coli even at low concentrations [174]. Silica nanoparticles functionalized with quaternary ammonium silane inhibited the growth of Gram-negative bacteria due to the synergistic effect of hydrophobicity and antibacterial activity [175]. QACs with $N$-halamine coated onto cotton fibers were active against $S$. aureus $[176,177]$. Similarly, the combination of these biocides was highly effective in macroporous cross-linked antimicrobial polymeric resin [160]. An antibacterial coating of immobilized QACs tethered on hyperbranched polyuria demonstrated high contact-killing efficacies toward adhering staphylococci [178]. Antimicrobial acrylic coatings with a QAC-containing perfluoroalkyl monomer were synthesized by using a self-stratification strategy via one-step UV curing [179]. Polyvinylidene fluoride membranes modified by QACs possess antibiofouling effects [180]. Bacterial cellulose incorporated with QACs showed strong and long-term antimicrobial activity against S. aureus and S. epidermidis [181]. QAC-based silver nanocomposites demonstrated synergistic antibiofilm properties along with a low hemolysis rate [182]. More examples of QACs immobilized on material surfaces with antibacterial activities can be found elsewhere $[45,47,49,159]$.

\subsubsection{Ionic Liquid-Containing Bactericidal Coatings}

Usage in bactericidal surface coatings seems one of the most promising applications of antibacterial ILs in medicine and other areas. Thus, the number of publications on the topic has been increasing steadily in recent years. As already mentioned above, ILs are proposed to be used as components of ionogels, films, and membranes that demonstrate considerable antimicrobial and antifouling activities (see, e.g., [89,93,163]). Cellulose nanofibers grafted with ammonium ILs and silver ions demonstrated significant antimicrobial activity against $S$. aureus MRSA and E. coli [183]. Zinc ion-coordinated poly-IL membranes with bactericidal properties were efficiently used for wound healing [184]. A conductive hydrogel wound dressing composed of a poly-IL (1-vinyl-3(aminopropyl)imidazolium tetrafluoroborate) and konjac glucomannan demonstrated long-lasting bactericidal activity against S. aureus and E. coli [185]. Similarly, promising results were obtained with a poly-IL (1-vinyl-3-butylimidazolium bromide)/poly(vinyl alcohol) wound dressing [172], a reusable 1-vinyl-3-butylimidazolium bromide-grafted cotton gauze wound dressing [173], and molecular brushes with 3-(12-mercaptododecyl)-1methylimidazolium bromide [186]. Composite membranes composed of bacterial cellulose and cholinium poly-ILs with amino acid anions were active against Gram-negative and Gram-positive bacteria and fungi [187]. Poly(vinylidene fluoride) (PVDF) materials grafted with ILs (1-vinyl-3-butylimidazolium chloride, 1-vinyl-3-ethylimidazolium tetrafluoroborate) showed activity against both common bacteria and "superbugs" [188]. Calcium phosphate-IL (1-alkyl-3-methylimidazolium chloride) materials with bactericidal properties were proposed to be used for implants [189]. Halloysite nanotubes functionalized with various ILs demonstrated antimicrobial activity [190].Coatings based on dicationic imidazolium ILs efficiently inhibited bacterial growth on titanium surfaces [191]. $\mathrm{TiO}_{2}$ nanomaterials coated with poly-IL brushes on the basis of imidazolium ILs demonstrated 
antibacterial and antifouling properties [192]. Cholinium salicylate-containing gelatin films with bactericidal activity were proposed to be used in food packaging [193]. In addition, 1-butyl-3-methylimidazolium bis(trifluoromethanesulfonyl)imide ([C $\left.\left.\mathrm{C}_{4} \mathrm{Mim}\right]\left[\mathrm{NTf}_{2}\right]\right)$ was tested as a bactericidal additive in orthodontic adhesive and was shown to reduce biofilm formation [194].

\section{Conclusions}

Despite the vast diversity of the available QAC structures, there are certain structural criteria designating the biocidal activity of the compounds.

Usually, the optimal alkyl tail length is within $\mathrm{C}_{10}-\mathrm{C}_{14}$; it can vary depending on the number of charges: $C_{12}$ and longer for mono-QACs and $C_{10}-C_{12}$ for bis-QACs. Nevertheless, in some series of compounds, those with tails of $\mathrm{C}_{10}$ and shorter demonstrated the highest activity. This observation suggests that the optimal chain length is specific for each set of structures and is related to the other fragments of the molecule.

In general, QACs with two or more charges (bis-QACs, multi-QACs, poly-QACs) have superior biocidal effects compared to mono-QACs. Moreover, many mono-QACs show little or no activity against Gram-negative bacteria. However, the addition of the second charged nitrogen without an alkyl chain does not always increase the activity, whereas the addition of the second and third alkyl chains increases the toxicity. The introduction of ether or amide bridges into QACs decreases both the toxicity and activity of the corresponding substances.

The combination of two bactericidal fragments with different mechanisms of action in one QAC has been proven to be a successful approach. These biocides have antibacterial and antifungal effects on a wide range of pathogens.

The assessment of the direct relation between the presence of aromatic and heterocyclic fragments/substituents in QAC molecules and their activity is complicated because this factor is highly specific for some structures. Relatively speaking, pyridine QACs, especially bis-pyridine salts with broad antibacterial/antifungal activity, are the most advanced and promising among all heterocyclic QACs. Aromatic structures are often used in QACs due to their strong reactivity. They can be spacers, substituents, tails, head parts, etc.

In 2016, in his report on antibacterial resistance, $\mathrm{O}^{\prime}$ Neill predicted that by 2050, $10 \mathrm{mil}-$ lion people would die because of resistant bacteria annually [195]. Moreover, SARS-CoV-2 aggravated the issue. During the current pandemic, antibacterial drugs are being used rather indiscriminately. It should be expected that the threat from resistant bacteria will increase significantly in the next few years. To avert this danger, the next generation of antibacterial drugs, including QACs, should be developed in the near future.

In this review, we analyze some of the structure-activity dependences and provide a general overview of the current situation in the research on antimicrobial QACs. In addition, a brief overview of the antimicrobial activities of various subclasses of ionic liquids, which are often considered advantageous antimicrobial agents, is also provided. We hope that it will serve as a highlight for future studies on these classes of biocides.

Supplementary Materials: The Supplementary Materials are available online at https:/ /www.mdpi. com/article/10.3390/ijms22136793/s1.

Funding: This research received no external funding.

Conflicts of Interest: The authors declare no conflict of interest.

\section{References}

1. Paulson, D.S. Topical Antimicrobials. In New Biocides Development; American Chemical Society: Washington, DC, USA, 2007; Volume 967, pp. 124-150.

2. Zheng, G.; Filippelli, G.M.; Salamova, A. Increased Indoor Exposure to Commonly Used Disinfectants during the COVID-19 Pandemic. Environ. Sci. Technol. Lett. 2020, 7, 760-765. [CrossRef]

3. Schrank, C.L.; Minbiole, K.P.C.; Wuest, W.M. Are Quaternary Ammonium Compounds, the Workhorse Disinfectants, Effective against Severe Acute Respiratory Syndrome-Coronavirus-2? ACS Infect. Dis. 2020, 6, 1553-1557. [CrossRef] [PubMed] 
4. Jacobs, W.A. The Bactericidal Properties of The Quaternary Salts of Hexamethylenetetramine: I. The Problem of The Chemotherapy of Experimental Bacterial Infections. J. Exp. Med. 1916, 23, 563-568. [CrossRef] [PubMed]

5. Jacobs, W.A.; Heidelberger, M.; Amoss, H.L. The Bactericidal Properties of The Quaternary Salts of Hexamethylenetetramine: II. The Relation Between Constitution and Bactericidal Action in the Substituted Benzylhexamethylenetetraminium. Salts. J. Exp. Med. 1916, 23, 569-576. [CrossRef] [PubMed]

6. Jacobs, W.A.; Heidelberger, M.; Bull, C.G. The Bactericidal Properties of The Quaternary Salts of Hexamethylenetetramine: III. The Relation Between Constitution And Bactericidal Action in the Quaternary Salts Obtained From Halogenacetyl Compounds. J. Exp. Med. 1916, 23, 577-599. [CrossRef]

7. Domagk, G. A new class of disinfectants. Dtsch. Med. Wochenschr 1935, 61, 829-832. [CrossRef]

8. Jennings, M.C.; Minbiole, K.P.C.; Wuest, W.M. Quaternary Ammonium Compounds: An Antimicrobial Mainstay and Platform for Innovation to Address Bacterial Resistance. ACS Infect. Dis. 2015, 1, 288-303. [CrossRef]

9. Directive, E.C. 98/8/EC of the European Parliament and of the Council of 16 February 1998 concerning the placing of biocidal products on the market. OJEC 1998, 123, 1-63.

10. Biocides Market Size, Share \& Trends Analysis Report by Product (Halogen Compounds, Quaternary Ammonium Compounds), By Application (Paints \& Coatings, Water Treatment), By Region, And Segment Forecasts, 2020-2027. Available online: www. grandviewresearch.com/industry-analysis/biocides-industry (accessed on 11 January 2021).

11. Gerba, C.P. Quaternary Ammonium Biocides: Efficacy in Application. Appl. Environ. Microbiol. 2015, 81, 464-469. [CrossRef]

12. Egorova, K.S.; Gordeev, E.G.; Ananikov, V.P. Biological activity of ionic liquids and their application in pharmaceutics and medicine. Chem. Rev. 2017, 117, 7132-7189. [CrossRef]

13. Simões, M.; Pereira, A.R.; Simões, L.C.; Cagide, F.; Borges, F. Biofilm control by ionic liquids. Drug Discov. Today 2021, 26, 1340-1346. [CrossRef]

14. Reregistration Eligibility Decision for Alkyl Dimethyl Benzyl Ammonium Chloride (ADBAC). In EPA 739-R-06-009; National Service Center for Enviromental Publications (NSCEP): Washington, DC, USA, 17 November 2006.

15. Rahn, O.; Eseltine, W.P.V. Quaternary Ammonium Compounds. Annu. Rev. Microbiol. 1947, 1, 173-192. [CrossRef]

16. De Saint Jean, M.; Brignole, F.; Bringuier, A.F.; Bauchet, A.; Feldmann, G.; Baudouin, C. Effects of benzalkonium chloride on growth and survival of Chang conjunctival cells. Investig. Ophthalmol. Vis. Sci. 1999, 40, 619-630.

17. Percival, S.L.; Finnegan, S.; Donelli, G.; Vuotto, C.; Rimmer, S.; Lipsky, B.A. Antiseptics for treating infected wounds: Efficacy on biofilms and effect of pH. Crit. Rev. Microbiol. 2016, 42, 293-309. [CrossRef] [PubMed]

18. Ogilvie, B.H.; Solis-Leal, A.; Lopez, J.B.; Poole, B.D.; Robison, R.A.; Berges, B.K. Alcohol-free hand sanitizer and other quaternary ammonium disinfectants quickly and effectively inactivate SARS-CoV-2. J. Hosp. Inf. 2021, 108, 142-145. [CrossRef] [PubMed]

19. Agafonova, M.N.; Kazakova, R.R.; Lubina, A.P.; Zeldi, M.I.; Nikitina, E.V.; Balakin, K.V.; Shtyrlin, Y.G. Antibacterial activity profile of miramistin in in vitro and in vivo models. Microb. Pathog. 2020, 142, 104072. [CrossRef] [PubMed]

20. Turov, V.V.; Barvinchenko, V.N.; Lipkovska, N.A.; Fedyanina, T.V. Supramolecular Structures in Nanosilica/Miramistin Hydrated Composite in a Hydrophobic Medium. J. Appl. Spectrosc. 2015, 82, 175-181. [CrossRef]

21. Grishin, M.N. [Use of antiseptic myramistin in the multimodality treatment of nonspecific suppurative pleuropulmonary diseases]. Probl. Tuberk. 1998, 1, 40-41.

22. Vertelov, G.K.; Krutyakov, Y.A.; Efremenkova, O.V.; Olenin, A.Y.; Lisichkin, G.V. A versatile synthesis of highly bactericidal Myramistin®stabilized silver nanoparticles. Nanotechnology 2008, 19, 355707. [CrossRef]

23. Quisno, R.; Foter, M.J. Cetyl Pyridinium Chloride: I. Germicidal Properties. J. Bacteriol. 1946, 52, 111-117. [CrossRef]

24. Mao, X.; Auer, D.L.; Buchalla, W.; Hiller, K.-A.; Maisch, T.; Hellwig, E.; Al-Ahmad, A.; Cieplik, F. Cetylpyridinium Chloride: Mechanism of Action, Antimicrobial Efficacy in Biofilms, and Potential Risks of Resistance. Antimicrob. Agents Chemother. 2020, 64, e00576-20. [CrossRef]

25. Bailey, D.M.; DeGrazia, C.G.; Hoff, S.J.; Schulenberg, P.L.; O'Connor, J.R.; Paris, D.A.; Slee, A.M. Bispyridinamines: A new class of topical antimicrobial agents as inhibitors of dental plaque. J. Med. Chem. 1984, 27, 1457-1464. [CrossRef] [PubMed]

26. Hübner, N.O.; Siebert, J.; Kramer, A. Octenidine Dihydrochloride, a Modern Antiseptic for Skin, Mucous Membranes and Wounds. Ski. Pharm. Phys. 2010, 23, 244-258. [CrossRef] [PubMed]

27. Stahl, J.; Braun, M.; Siebert, J.; Kietzmann, M. The percutaneous permeation of a combination of $0.1 \%$ octenidine dihydrochloride and $2 \%$ 2-phenoxyethanol (octenisept ${ }^{\circledR}$ ) through skin of different species in vitro. BMC Vet. Res. 2011, 7, 44. [CrossRef]

28. Cherian, B.; Gehlot, P.M.; Manjunath, M.K. Comparison of the Antimicrobial Efficacy of Octenidine Dihydrochloride and Chlorhexidine with and Without Passive Ultrasonic Irrigation-An Invitro Study. J. Clin. Diagn. Res. 2016, 10, ZC71-ZC77. [CrossRef] [PubMed]

29. Dettenkofer, M.; Wilson, C.; Gratwohl, A.; Schmoor, C.; Bertz, H.; Frei, R.; Heim, D.; Luft, D.; Schulz, S.; Widmer, A.F. Skin disinfection with octenidine dihydrochloride for central venous catheter site care: A double-blind, randomized, controlled trial. Clin. Microbiol. Infect. 2010, 16, 600-606. [CrossRef] [PubMed]

30. Hadaway, L. Polyhexamethylene Biguanide Dressing-Another Promising Tool to Reduce Catheter-related Bloodstream Infection. JAVA 2010, 15, 203-205. [CrossRef]

31. Roberts, W.R.; Addy, M. Comparison of the in vivo and in vitro antibacterial properties of antiseptic mouthrinses containing chlorhexidine, alexidine, cetyl pyridinium chloride and hexetidine. J. Clin. Periodontol. 1981, 8, 295-310. [CrossRef] 
32. Gilbert, P.; Moore, L.E. Cationic antiseptics: Diversity of action under a common epithet. J. Appl. Microbiol. $2005,99,703-715$. [CrossRef]

33. Hope, C.K.; Wilson, M. Analysis of the Effects of Chlorhexidine on Oral Biofilm Vitality and Structure Based on Viability Profiling and an Indicator of Membrane Integrity. Antimicrob. Agents Chemother. 2004, 48, 1461-1468. [CrossRef]

34. Thomas, B.; Stickler, D.J. Chlorhexidine resistance and the lipids of Providencia stuartii. Microbios 1979, 24, 141-150.

35. Moore, K.; Gray, D. Using PHMB antimicrobial to prevent wound infection. Wounds UK 2007, 3, 96-102.

36. Allen, M.J.; White, G.F.; Morby, A.P. The response of Escherichia coli to exposure to the biocide polyhexamethylene biguanide. Microbiology 2006, 152, 989-1000. [CrossRef] [PubMed]

37. Zhou, C.; Wang, Y. Structure-activity relationship of cationic surfactants as antimicrobial agents. Curr. Opin. Colloid Interface Sci. 2020, 45, 28-43. [CrossRef]

38. Vereshchagin, A.N. Classical and interdisciplinary approaches to the design of organic and hybrid molecular systems. Russ. Chem. Bull. 2017, 66, 1765-1796. [CrossRef]

39. Brown, A.C.; Fraser, T.R. On the Connection between Chemical Constitution and Physiological Action; with special reference to the Physiological Action of the Salts of the Ammonium Bases derived from Strychnia, Brucia, Thebaia, Codeia, Morphia, and Nicotia. J. Anat. Physiol. 1868, 2, 224-242. [PubMed]

40. Roy, K.; Kar, S.; Das, R.N. A Primer on QSAR/QSPR Modeling; Springer International Publishing: Berlin/Heidelberg, Germany, 2015.

41. Obłak, E.; Piecuch, A.; Rewak-Soroczyńska, J.; Paluch, E. Activity of gemini quaternary ammonium salts against microorganisms. Appl. Microbiol. Biotechnol. 2019, 103, 625-632. [CrossRef]

42. Tischer, M.; Pradel, G.; Ohlsen, K.; Holzgrabe, U. Quaternary Ammonium Salts and Their Antimicrobial Potential: Targets or Nonspecific Interactions? Chem. Med. Chem. 2012, 7, 22-31. [CrossRef]

43. Thorsteinsson, T.; Loftsson, T.; Masson, M. Soft Antibacterial Agents. Curr. Med. Chem. 2003, 10, 1129-1136. [CrossRef]

44. Zubris, D.L.; Minbiole, K.P.C.; Wuest, W.M. Polymeric Quaternary Ammonium Compounds: Versatile Antimicrobial Materials. Curr. Top. Med. Chem. 2017, 17, 305-318. [CrossRef]

45. Makvandi, P.; Jamaledin, R.; Jabbari, M.; Nikfarjam, N.; Borzacchiello, A. Antibacterial quaternary ammonium compounds in dental materials: A systematic review. Dent. Mater. 2018, 34, 851-867. [CrossRef]

46. Andreica, B.-I.; Cheng, X.; Marin, L. Quaternary ammonium salts of chitosan. A critical overview on the synthesis and properties generated by quaternization. Eur. Polym. J. 2020, 139, 110016. [CrossRef]

47. Xue, Y.; Xiao, H.; Zhang, Y. Antimicrobial Polymeric Materials with Quaternary Ammonium and Phosphonium Salts. Int. J. Mol. Sci. 2015, 16, 3626-3655. [CrossRef]

48. Sowmiah, S.; Esperança, J.M.S.S.; Rebelo, L.P.N.; Afonso, C.A.M. Pyridinium salts: From synthesis to reactivity and applications. Org. Chem. Front. 2018, 5, 453-493. [CrossRef]

49. Jiao, Y.; Niu, L.-N.; Ma, S.; Li, J.; Tay, F.R.; Chen, J.-H. Quaternary ammonium-based biomedical materials: State-of-the-art, toxicological aspects and antimicrobial resistance. Prog. Polym. Sci. 2017, 71, 53-90. [CrossRef] [PubMed]

50. Muñoz-Bonilla, A.; Fernández-García, M. Polymeric materials with antimicrobial activity. Prog. Polym. Sci. 2012, 37, 281-339. [CrossRef]

51. Bureš, F. Quaternary Ammonium Compounds: Simple in Structure, Complex in Application. Top. Curr. Chem. 2019, $377,14$. [CrossRef] [PubMed]

52. Thorsteinsson, T.; Másson, M.; Kristinsson, K.G.; Hjálmarsdóttir, M.A.; Hilmarsson, H.; Loftsson, T. Soft Antimicrobial Agents: Synthesis and Activity of Labile Environmentally Friendly Long Chain Quaternary Ammonium Compounds. J. Med. Chem. 2003, 46, 4173-4181. [CrossRef] [PubMed]

53. Mikláš, R.; Miklášová, N.; Bukovský, M.; Devínsky, F. Synthesis and antimicrobial properties of camphorsulfonic acid derived imidazolium salts. Acta Fac. Pharm. Univ. Comen. 2014, 61, 42-48. [CrossRef]

54. Mikláš, R.; Miklášová, N.; Bukovský, M.; Horváth, B.; Kubincová, J.; Devínsky, F. Synthesis, surface and antimicrobial properties of some quaternary ammonium homochiral camphor sulfonamides. Eur. J. Pharm. Sci. 2014, 65, 29-37. [CrossRef] [PubMed]

55. Ali, I.; Burki, S.; El-Haj, B.M.; Shafiullah; Parveen, S.; Nadeem, H.Ş.; Nadeem, S.; Shah, M.R. Synthesis and characterization of pyridine-based organic salts: Their antibacterial, antibiofilm and wound healing activities. Bioorg. Chem. 2020, 100, 103937. [CrossRef]

56. Li, L.; Pu, T.; Zhanel, G.; Zhao, N.; Ens, W.; Liu, S. New Biocide with Both N-Chloramine and Quaternary Ammonium Moieties Exerts Enhanced Bactericidal Activity. Adv. Health. Mater. 2012, 1, 609-620. [CrossRef] [PubMed]

57. Ning, C.; Li, L.; Logsetty, S.; Ghanbar, S.; Guo, M.; Ens, W.; Liu, S. Enhanced antibacterial activity of new "composite" biocides with both N-chloramine and quaternary ammonium moieties. Rsc Adv. 2015, 5, 93877-93887. [CrossRef]

58. Ghanbar, S.; Kazemian, M.R.; Liu, S. New Generation of N-Chloramine/QAC Composite Biocides: Efficient Antimicrobial Agents To Target Antibiotic-Resistant Bacteria in the Presence of Organic Load. ACS Omega 2018, 3, 9699-9709. [CrossRef] [PubMed]

59. Li, L.; Zhao, Y.; Zhou, H.; Ning, A.; Zhang, F.; Zhao, Z. Synthesis of pyridinium N-chloramines for antibacterial applications. Tetrahedron Lett. 2017, 58, 321-325. [CrossRef]

60. Liu, W.-S.; Wang, C.-H.; Sun, J.-F.; Hou, G.-G.; Wang, Y.-P.; Qu, R.-J. Synthesis, Characterization and Antibacterial Properties of Dihydroxy Quaternary Ammonium Salts with Long Chain Alkyl Bromides. Chem. Biol. Drug Des. 2015, 85, 91-97. [CrossRef]

61. Xie, X.; Cong, W.; Zhao, F.; Li, H.; Xin, W.; Hou, G.; Wang, C. Synthesis, physiochemical property and antimicrobial activity of novel quaternary ammonium salts. J. Enzym. Inhib. Med. Chem. 2018, 33, 98-105. [CrossRef] [PubMed] 
62. Bogdanov, A.V.; Zaripova, I.F.; Voloshina, A.D.; Sapunova, A.S.; Kulik, N.V.; Bukharov, S.V.; Voronina, J.K.; Vandyukov, A.E.; Mironov, V.F. Synthesis and Biological Evaluation of New Isatin-Based QACs with High Antimicrobial Potency. Chem. Sel. 2019, 4, 6162-6166. [CrossRef]

63. Rusew, R.; Kurteva, V.; Shivachev, B. Novel Quaternary Ammonium Derivatives of 4-Pyrrolidino Pyridine: Synthesis, Structural, Thermal, and Antibacterial Studies. Crystals 2020, 10, 339. [CrossRef]

64. Salajkova, S.; Benkova, M.; Marek, J.; Sleha, R.; Prchal, L.; Malinak, D.; Dolezal, R.; Sepčić, K.; Gunde-Cimerman, N.; Kuca, K.; et al. Wide-Antimicrobial Spectrum of Picolinium Salts. Molecules 2020, 25, 2254. [CrossRef] [PubMed]

65. Shtyrlin, N.V.; Sapozhnikov, S.V.; Koshkin, S.A.; Iksanova, A.G.; Sabirov, A.H.; Kayumov, A.R.; Nureeva, A.A.; Zeldi, M.I.; Shtyrlin, Y.G. Synthesis and Antibacterial Activity of Novel Quaternary Ammonium Pyridoxine Derivatives. Med. Chem. 2015, 11, 656-665. [CrossRef] [PubMed]

66. Sapozhnikov, S.V.; Shtyrlin, N.V.; Kayumov, A.R.; Zamaldinova, A.E.; Iksanova, A.G.; Nikitina, E.V.; Krylova, E.S.; Grishaev, D.Y.; Balakin, K.V.; Shtyrlin, Y.G. New quaternary ammonium pyridoxine derivatives: Synthesis and antibacterial activity. Med. Chem. Res. 2017, 26, 3188-3202. [CrossRef]

67. Kayumov, A.R.; Nureeva, A.A.; Trizna, E.Y.; Gazizova, G.R.; Bogachev, M.I.; Shtyrlin, N.V.; Pugachev, M.V.; Sapozhnikov, S.V.; Shtyrlin, Y.G. New Derivatives of Pyridoxine Exhibit High Antibacterial Activity against Biofilm-Embedded Staphylococcus Cells. Biomed Res. Int. 2015, 2015, 890968. [CrossRef] [PubMed]

68. Shtyrlin, N.V.; Sapozhnikov, S.V.; Galiullina, A.S.; Kayumov, A.R.; Bondar, O.V.; Mirchink, E.P.; Isakova, E.B.; Firsov, A.A.; Balakin, K.V.; Shtyrlin, Y.G. Synthesis and Antibacterial Activity of Quaternary Ammonium 4-Deoxypyridoxine Derivatives. Biomed Res. Int. 2016, 2016, 3864193. [CrossRef] [PubMed]

69. Garipov, M.R.; Sabirova, A.E.; Pavelyev, R.S.; Shtyrlin, N.V.; Lisovskaya, S.A.; Bondar, O.V.; Laikov, A.V.; Romanova, J.G.; Bogachev, M.I.; Kayumov, A.R.; et al. Targeting pathogenic fungi, bacteria and fungal-bacterial biofilms by newly synthesized quaternary ammonium derivative of pyridoxine and terbinafine with dual action profile. Bioorg. Chem. 2020, $104,104306$. [CrossRef]

70. Sapozhnikov, S.V.; Sabirova, A.E.; Shtyrlin, N.V.; Druk, A.Y.; Agafonova, M.N.; Chirkova, M.N.; Kazakova, R.R.; Grishaev, D.Y.; Nikishova, T.V.; Krylova, E.S.; et al. Design, synthesis, antibacterial activity and toxicity of novel quaternary ammonium compounds based on pyridoxine and fatty acids. Eur. J. Med. Chem. 2021, 211, 113100. [CrossRef] [PubMed]

71. Paniak, T.J.; Jennings, M.C.; Shanahan, P.C.; Joyce, M.D.; Santiago, C.N.; Wuest, W.M.; Minbiole, K.P.C. The antimicrobial activity of mono-, bis-, tris-, and tetracationic amphiphiles derived from simple polyamine platforms. Bioorg. Med. Chem. Lett. 2014, 24, 5824-5828. [CrossRef]

72. Mitchell, M.A.; Iannetta, A.A.; Jennings, M.C.; Fletcher, M.H.; Wuest, W.M.; Minbiole, K.P.C. Scaffold-Hopping of Multicationic Amphiphiles Yields Three New Classes of Antimicrobials. Chem. Bio. Chem. 2015, 16, 2299-2303. [CrossRef]

73. Minbiole, K.P.C.; Jennings, M.C.; Ator, L.E.; Black, J.W.; Grenier, M.C.; LaDow, J.E.; Caran, K.L.; Seifert, K.; Wuest, W.M. From antimicrobial activity to mechanism of resistance: The multifaceted role of simple quaternary ammonium compounds in bacterial eradication. Tetrahedron 2016, 72, 3559-3566. [CrossRef]

74. Joyce, M.D.; Jennings, M.C.; Santiago, C.N.; Fletcher, M.H.; Wuest, W.M.; Minbiole, K.P.C. Natural product-derived quaternary ammonium compounds with potent antimicrobial activity. J. Antibiot. 2016, 69, 344-347. [CrossRef]

75. Black, J.W.; Jennings, M.C.; Azarewicz, J.; Paniak, T.J.; Grenier, M.C.; Wuest, W.M.; Minbiole, K.P.C. TMEDA-derived biscationic amphiphiles: An economical preparation of potent antibacterial agents. Bioorg. Med. Chem. Lett. 2014, 24, 99-102. [CrossRef]

76. Allen, R.A.; Jennings, M.C.; Mitchell, M.A.; Al-Khalifa, S.E.; Wuest, W.M.; Minbiole, K.P.C. Ester- and amide-containing multiQACs: Exploring multicationic soft antimicrobial agents. Bioorg. Med. Chem. Lett. 2017, 27, 2107-2112. [CrossRef]

77. Hayes, R.; Warr, G.G.; Atkin, R. Structure and nanostructure in ionic liquids. Chem. Rev. 2015, 115, 6357-6426. [CrossRef]

78. Egorova, K.S.; Ananikov, V.P. Toxicity of ionic liquids: Eco(cyto)activity as complicated, but unavoidable parameter for taskspecific optimization. Chem. Sus. Chem. 2014, 7, 336-360. [CrossRef]

79. Egorova, K.S.; Ananikov, V.P. Fundamental importance of ionic interactions in the liquid phase: A review of recent studies of ionic liquids in biomedical and pharmaceutical applications. J. Mol. Liq. 2018, 272, 271-300. [CrossRef]

80. Moshikur, R.; Chowdhury, R.; Moniruzzaman, M.; Goto, M. Biocompatible ionic liquids and their applications in pharmaceutics. Green Chem. 2020, 22, 8116-8139. [CrossRef]

81. Demberelnyamba, D.; Kim, K.-S.; Choi, S.; Park, S.-Y.; Lee, H.; Kim, C.-J.; Yoo, I.-D. Synthesis and antimicrobial properties of imidazolium and pyrrolidinonium salts. Bioorg. Med. Chem. Lett. 2004, 12, 853-857. [CrossRef]

82. Ferraz, R.; Teixeira, V.; Rodrigues, D.; Fernandes, R.; Prudêncio, C.; Noronha, J.P.; Petrovski, Ž.; Branco, L.C. Antibacterial activity of ionic liquids based on ampicillin against resistant bacteria. RSC Adv. 2014, 4, 4301-4307. [CrossRef]

83. Ferraz, R.; Branco, L.C.; Prudêncio, C.; Noronha, J.P.; Petrovski, Ž. Ionic liquids as active pharmaceutical ingredients. ChemMedChem 2011, 6, 975-985. [CrossRef] [PubMed]

84. Prudêncio, C.; Vieira, M.; Van der Auweraer, S.; Ferraz, R. Recycling old antibiotics with ionic liquids. Antibiotics 2020, 9, 578. [CrossRef] [PubMed]

85. Carson, L.; Chau, P.K.W.; Earle, M.J.; Gilea, M.A.; Gilmore, B.F.; Gorman, S.P.; McCann, M.T.; Seddon, K.R. Antibiofilm activities of 1-alkyl-3-methylimidazolium chloride ionic liquids. Green Chem. 2009, 11, 492-497. [CrossRef]

86. Gundolf, T.; Rauch, B.; Kalb, R.; Rossmanith, P.; Mester, P. Influence of bacterial lipopolysaccharide modifications on the efficacy of antimicrobial ionic liquids. J. Mol. Liq. 2018, 271, 220-227. [CrossRef] 
87. Cornellas, A.; Perez, L.; Comelles, F.; Ribosa, I.; Manresa, A.; Garcia, M.T. Self-aggregation and antimicrobial activity of imidazolium and pyridinium based ionic liquids in aqueous solution. J. Colloid Interface Sci. 2011, 355, 164-171. [CrossRef]

88. Bergamo, V.Z.; Donato, R.K.; Dalla Lana, D.F.; Donato, K.J.Z.; Ortega, G.G.; Schrekker, H.S.; Fuentefria, A.M. Imidazolium salts as antifungal agents: Strong antibiofilm activity against multidrug-resistant Candida tropicalis isolates. Lett. Appl. Microbiol. 2015, 60, 66-71. [CrossRef]

89. Qin, J.; Guo, J.; Xu, Q.; Zheng, Z.; Mao, H.; Yan, F. Synthesis of pyrrolidinium-type poly(ionic liquid) membranes for antibacterial applications. ACS Appl. Mater. Interfaces 2017, 9, 10504-10511. [CrossRef]

90. Florio, W.; Becherini, S.; D'Andrea, F.; Lupetti, A.; Chiappe, C.; Guazzelli, L. Comparative evaluation of antimicrobial activity of different types of ionic liquids. Mater. Sci. Eng. C 2019, 104, 109907. [CrossRef] [PubMed]

91. Florio, W.; Rizzato, C.; Becherini, S.; Guazzelli, L.; D'Andrea, F.; Lupetti, A. Synergistic activity between colistin and the ionic liquids 1-methyl-3-dodecylimidazolium bromide, 1-dodecyl-1-methylpyrrolidinium bromide, or 1-dodecyl-1-methylpiperidinium bromide against Gram-negative bacteria. J. Glob. Antimicrob. Resist. 2020, 21, 99-104. [CrossRef] [PubMed]

92. Siopa, F.; Figueiredo, T.; Frade, R.F.M.; Neto, I.; Meirinhos, A.; Reis, C.P.; Sobral, R.G.; Afonso, C.A.M.; Rijo, P. Choline-based ionic liquids: Improvement of antimicrobial activity. Chem. Sel. 2016, 1, 5909-5916. [CrossRef]

93. De Leo, F.; Marchetta, A.; Capillo, G.; Germanà, A.; Primerano, P.; Schiavo, S.L.; Urzì, C. Surface active ionic liquids based coatings as subaerial anti-biofilms for stone built cultural heritage. Coatings 2020, 11, 26. [CrossRef]

94. Hajfarajollah, H.; Mokhtarani, B.; Noghabi, K.A.; Sharifi, A.; Mirzaei, M. Antibacterial and antiadhesive properties of butylmethylimidazolium ionic liquids toward pathogenic bacteria. Rsc Adv. 2014, 4, 42751-42757. [CrossRef]

95. Anvari, S.; Hajfarajollah, H.; Mokhtarani, B.; Enayati, M.; Sharifi, A.; Mirzaei, M. Antibacterial and anti-adhesive properties of ionic liquids with various cationic and anionic heads toward pathogenic bacteria. J. Mol. Liq. 2016, 221, 685-690. [CrossRef]

96. Weyhing-Zerrer, N.; Kalb, R.; Oßmer, R.; Rossmanith, P.; Mester, P. Evidence of a reverse side-chain effect of tris(pentafluoroethyl)tri fluorophosphate [FAP]-based ionic liquids against pathogenic bacteria. Ecotoxicol. Environ. Saf. 2018, 148, 467-472. [CrossRef]

97. Cole, M.R.; Li, M.; El-Zahab, B.; Janes, M.E.; Hayes, D.; Warner, I.M. Design, synthesis, and biological evaluation of $\beta$-lactam antibiotic-based imidazolium- and pyridinium-type ionic liquids. Chem. Biol. Drug Des. 2011, 78, 33-41. [CrossRef]

98. Venkata Nancharaiah, Y.; Reddy, G.K.K.; Lalithamanasa, P.; Venugopalan, V.P. The ionic liquid 1-alkyl-3-methylimidazolium demonstrates comparable antimicrobial and antibiofilm behavior to a cationic surfactant. Biofouling 2012, 28, 1141-1149. [CrossRef]

99. Hough-Troutman, W.L.; Smiglak, M.; Griffin, S.; Matthew Reichert, W.; Mirska, I.; Jodynis-Liebert, J.; Adamska, T.; Nawrot, J.; Stasiewicz, M.; Rogers, R.D.; et al. Ionic liquids with dual biological function: Sweet and anti-microbial, hydrophobic quaternary ammonium-based salts. N. J. Chem. 2009, 33, 26-33. [CrossRef]

100. Menger, F.M.; Littau, C.A. Gemini surfactants: A new class of self-assembling molecules. J. Am. Chem. Soc. 1993, 115, 10083-10090. [CrossRef]

101. Pavlíková-Mořická, M.; Lacko, I.; Devínsky, F.; Masárová, L.; Mlynarčík, D. Quantitative relationships between structure and antimicrobial activity of new "Soft" bisquaternary ammonium salts. Fol. Microbiol. 1994, 39, 176-180. [CrossRef] [PubMed]

102. Devínsky, F.; Kopecka-Leitmanová, A.; Šeršeň, F.; Balgavý, P. Cut-off Effect in Antimicrobial Activity and in Membrane Perturbation Efficiency of the Homologous Series of N,N-Dimethylalkylamine Oxidest. J. Pharm. Pharm. 1990, 42, 790-794. [CrossRef] [PubMed]

103. Hoque, J.; Akkapeddi, P.; Yarlagadda, V.; Uppu, D.S.S.M.; Kumar, P.; Haldar, J. Cleavable Cationic Antibacterial Amphiphiles: Synthesis, Mechanism of Action, and Cytotoxicities. Langmuir 2012, 28, 12225-12234. [CrossRef] [PubMed]

104. Jennings, M.C.; Buttaro, B.A.; Minbiole, K.P.C.; Wuest, W.M. Bioorganic Investigation of Multicationic Antimicrobials to Combat QAC-Resistant Staphylococcus aureus. ACS Infect. Dis. 2015, 1, 304-309. [CrossRef] [PubMed]

105. LaDow, J.E.; Warnock, D.C.; Hamill, K.M.; Simmons, K.L.; Davis, R.W.; Schwantes, C.R.; Flaherty, D.C.; Willcox, J.A.L.; WilsonHenjum, K.; Caran, K.L.; et al. Bicephalic amphiphile architecture affects antibacterial activity. Eur. J. Med. Chem. 2011, 46, 4219-4226. [CrossRef]

106. Shtyrlin, N.V.; Pugachev, M.V.; Sapozhnikov, S.V.; Garipov, M.R.; Vafina, R.M.; Grishaev, D.Y.; Pavelyev, R.S.; Kazakova, R.R.; Agafonova, M.N.; Iksanova, A.G.; et al. Novel Bis-Ammonium Salts of Pyridoxine: Synthesis and Antimicrobial Properties. Molecules 2020, 25, 4341. [CrossRef] [PubMed]

107. Forman, M.E.; Fletcher, M.H.; Jennings, M.C.; Duggan, S.M.; Minbiole, K.P.C.; Wuest, W.M. Structure-Resistance Relationships: Interrogating Antiseptic Resistance in Bacteria with Multicationic Quaternary Ammonium Dyes. Chem. Med. Chem. 2016, 11, 958-962. [CrossRef] [PubMed]

108. Zhou, F.; Maeda, T.; Nagamune, H.; Kourai, H. Synthesis and Antimicrobial Characteristics of Novel Biocides, 1, 1'-(Decanedioyl) bis (4-methy1-4-alkylpiperazinium iodide) s with a Gemini Structure. Biocontrol Sci. 2004, 9, 61-67. [CrossRef]

109. Kontos, R.C.; Schallenhammer, S.A.; Bentley, B.S.; Morrison, K.R.; Feliciano, J.A.; Tasca, J.A.; Kaplan, A.R.; Bezpalko, M.W.; Kassel, W.S.; Wuest, W.M.; et al. An Investigation into Rigidity-Activity Relationships in BisQAC Amphiphilic Antiseptics. Chem. Med. Chem. 2019, 14, 83-87. [CrossRef] [PubMed]

110. Ma, J.; Liu, N.; Huang, M.; Wang, L.; Han, J.; Qian, H.; Che, F. Synthesis, physicochemical and antimicrobial properties of cardanol-derived quaternary ammonium compounds (QACs) with heterocyclic polar head. J. Mol. Liq. 2019, $294,111669$. [CrossRef] 
111. Schallenhammer, S.A.; Duggan, S.M.; Morrison, K.R.; Bentley, B.S.; Wuest, W.M.; Minbiole, K.P.C. Hybrid BisQACs: Potent Biscationic Quaternary Ammonium Compounds Merging the Structures of Two Commercial Antiseptics. Chem. Med. Chem. 2017, 12, 1931-1934. [CrossRef]

112. Morrison, K.R.; Allen, R.A.; Minbiole, K.P.C.; Wuest, W.M. More QACs, more questions: Recent advances in structure activity relationships and hurdles in understanding resistance mechanisms. Tetrahedron Lett. 2019, 60, 150935. [CrossRef]

113. Thomas, B.; Duval, R.E.; Fontanay, S.; Varbanov, M.; Boisbrun, M. Synthesis and Antibacterial Evaluation of Bis-thiazolium, Bis-imidazolium, and Bis-triazolium Derivatives. Chem. Med. Chem. 2019, 14, 1232-1237. [CrossRef]

114. Shirai, A.; Sumitomo, T.; Yoshida, M.; Kaimura, T.; Nagamune, H.; Maeda, T.; Kourai, H. Synthesis and Biological Properties of Gemini Quaternary Ammonium Compounds, 5,5'-[2,2'-(alpha,omega-Polymethylnedicarbonyldioxy)diethyl]bis-(3-alkyl-4methylthiazolium iodide) and 5,5'-[2,2'-( $p$-Phenylenedicarbonyldioxy)diethyl]bis(3-alkyl-4-methylthiazolium bromide). Chem. Pharm. Bull. 2006, 54, 639-645.

115. Shrestha, J.P.; Baker, C.; Kawasaki, Y.; Subedi, Y.P.; Vincent de Paul, N.N.; Takemoto, J.Y.; Chang, C.-W.T. Synthesis and bioactivity investigation of quinone-based dimeric cationic triazolium amphiphiles selective against resistant fungal and bacterial pathogens. Eur. J. Med. Chem. 2017, 126, 696-704. [CrossRef]

116. Grenier, M.C.; Davis, R.W.; Wilson-Henjum, K.L.; LaDow, J.E.; Black, J.W.; Caran, K.L.; Seifert, K.; Minbiole, K.P.C. The antibacterial activity of $4,4^{\prime}$-bipyridinium amphiphiles with conventional, bicephalic and gemini architectures. Bioorg. Med. Chem. Lett. 2012, 22, 4055-4058. [CrossRef]

117. Ator, L.E.; Jennings, M.C.; McGettigan, A.R.; Paul, J.J.; Wuest, W.M.; Minbiole, K.P.C. Beyond paraquats: Dialkyl 3,3'- and 3,4'-bipyridinium amphiphiles as antibacterial agents. Bioorg. Med. Chem. Lett. 2014, 24, 3706-3709. [CrossRef] [PubMed]

118. Leitgeb, A.J.; Feliciano, J.A.; Sanchez, H.A.; Allen, R.A.; Morrison, K.R.; Sommers, K.J.; Carden, R.G.; Wuest, W.M.; Minbiole, K.P.C. Further Investigations into Rigidity-Activity Relationships in BisQAC Amphiphilic Antiseptics. Chem. Med. Chem. 2020, 15, 667-670. [CrossRef] [PubMed]

119. Tsuji, Y.; Yamamoto, M.; Vereshchagin, A.N.; Dorofeev, A.S.; Geyvandova, T.A.; Agafonova, I.F.; Geyvandov, R.K. Dimeric Quaternary Pyridinium Salts Possessing Biocidal Activity. Patent \#WO158045, 2 October 2014.

120. Yamamoto, M.; Takami, T.; Matsumura, R.; Dorofeev, A.; Hirata, Y.; Nagamune, H. In vitro evaluation of the biocompatibility of newly synthesized bis-quaternary ammonium compounds with spacer structures derived from pentaerythritol or hydroquinone. Biocontrol. Sci. 2016, 21, 231-241. [CrossRef]

121. Yamamoto, M.; Matsumura, R.; Hirata, Y.; Nagamune, H. A comparative study of skin irritation caused by novel bis-quaternary ammonium compounds and commonly used antiseptics by using cell culture methods. Toxicol. Vitr. 2019, 54, 75-81. [CrossRef]

122. Vereshchagin, A.N.; Gordeeva, A.M.; Frolov, N.A.; Proshin, P.I.; Hansford, K.A.; Egorov, M.P. Synthesis and Microbiological Properties of Novel Bis-Quaternary Ammonium Compounds Based on Biphenyl Spacer. Eur. J. Org. Chem 2019, $2019,4123-4127$. [CrossRef]

123. Vereshchagin, A.N.; Frolov, N.A.; Konyuhova, V.Y.; Hansford, K.A.; Egorov, M.P. Synthesis and microbiological properties of novel bis-quaternary ammonium compounds based on 4,4'-oxydiphenol spacer. Mendeleev Commun. 2019, 29, 523-525. [CrossRef]

124. Vereshchagin, A.N.; Frolov, N.A.; Konyuhova, V.Y.; Dorofeeva, E.O.; Hansford, K.A.; Egorov, M.P. Synthesis and biological evaluation of novel bis-quaternary ammonium compounds with p-terphenyl spacer. Mendeleev Commun. 2020, 30, 424-426. [CrossRef]

125. Vereshchagin, A.N.; Frolov, N.A.; Pakina, A.S.; Hansford, K.A.; Egorov, M.P. Synthesis and biological evaluation of novel bispyridinium salts containing naphthalene-2,7-diylbis(oxy) spacer. Mendeleev Commun. 2020, 30, 703-705. [CrossRef]

126. Vereshchagin, A.N.; Frolov, N.A.; Konyuhova, V.Y.; Kapelistaya, E.A.; Hansford, K.A.; Egorov, M.P. Investigations into the structure-activity relationship in gemini QACs based on biphenyl and oxydiphenyl linker. Rsc Adv. 2021, 11, 3429-3438 [CrossRef]

127. Shirai, A.; Maeda, T.; Hara, I.; Yoshinari, A.; Nagamune, H.; Kourai, H. Antimicrobial Characteristics of Bis-quaternary Ammonium Compounds Possessing a p-Phenylene Group in Their Spacer Chains. Biocontrol Sci. 2003, 8, 151-157. [CrossRef]

128. Sumitomo, T.; Maeda, T.; Nagamune, H.; Kourai, H. Bacterioclastic Action of a Bis-Quaternary Ammonium Compound against Escherichia coli. Biocontrol Sci. 2004, 9, 1-9. [CrossRef]

129. Yabuhara, T.; Maeda, T.; Nagamune, H.; Kourai, H. Synthesis and Antimicrobial Characteristics of a Novel Biocide, 4, 4'-(1, 6-Dioxyhexamethylene) bis-(1-alkylpyridinium halide). Biocontrol. Sci. 2004, 9, 95-103. [CrossRef]

130. Ohkura, K.; Sukeno, A.; Nagamune, H.; Kourai, H. Bridge-linked bis-quaternary ammonium anti-microbial agents: Relationship between cytotoxicity and anti-bacterial activity of 5,5'-[2,2'-(tetramethylenedicarbonyldioxy)-diethyl]bis(3-alkyl-4methylthiazonium iodide)s. Bioorg. Med. Chem. 2005, 13, 2579-2587. [CrossRef] [PubMed]

131. Kourai, H.; Yabuhara, T.; Shirai, A.; Maeda, T.; Nagamune, H. Syntheses and antimicrobial activities of a series of new bisquaternary ammonium compounds. Eur. J. Med. Chem. 2006, 41, 437-444. [CrossRef]

132. Murakami, K.; Yumoto, H.; Murakami, A.; Amoh, T.; Viducic, D.; Hirota, K.; Tabata, A.; Nagamune, H.; Kourai, H.; Matsuo, T.; et al. Evaluation of the effectiveness of the potent bis-quaternary ammonium compound, $4,4^{\prime}-(\alpha, \omega$-hexametylenedithio) bis (1-octylpyridinium bromide) (4DTBP-6,8) on Pseudomonas aeruginosa. J. Appl. Microbiol. 2017, 122, 893-899. [CrossRef]

133. Obando, D.; Koda, Y.; Pantarat, N.; Lev, S.; Zuo, X.; Bijosono Oei, J.; Widmer, F.; Djordjevic, J.T.; Sorrell, T.C.; Jolliffe, K.A. Synthesis and Evaluation of a Series of Bis(pentylpyridinium) Compounds as Antifungal Agents. Chem. Med. Chem. 2018, 13, 1421-1436. [CrossRef] [PubMed] 
134. Hao, J.; Qin, T.; Zhang, Y.; Li, Y.; Zhang, Y. Synthesis, surface properties and antimicrobial performance of novel gemini pyridinium surfactants. Colloids Surf. B 2019, 181, 814-821. [CrossRef]

135. Vereshchagin, A.N.; Karpenko, K.A.; Egorov, M.P. Synthesis and antibacterial activity of new dimeric pyridinium chlorides based on 2,2-bis(hydroxymethyl)propane-1,3-diyl spacer. Russ. Chem. Bull. 2020, 69, 620-623. [CrossRef]

136. Rezki, N.; Al-Sodies, S.A.; Ahmed, H.E.A.; Ihmaid, S.; Messali, M.; Ahmed, S.; Aouad, M.R. A novel dicationic ionic liquids encompassing pyridinium hydrazone-phenoxy conjugates as antimicrobial agents targeting diverse high resistant microbial strains. J. Mol. Liq. 2019, 284, 431-444. [CrossRef]

137. Gindri, I.M.; Siddiqui, D.A.; Bhardwaj, P.; Rodriguez, L.C.; Palmer, K.L.; Frizzo, C.P.; Martins, M.A.P.; Rodrigues, D.C. Dicationic imidazolium-based ionic liquids: A new strategy for non-toxic and antimicrobial materials. Rsc Adv. 2014, 4, 62594-62602. [CrossRef]

138. Ganapathi, P.; Ganesan, K.; Vijaykanth, N.; Arunagirinathan, N. Anti-bacterial screening of water soluble carbonyl diimidazolium salts and its derivatives. J. Mol. Liq. 2016, 219, 180-185. [CrossRef]

139. Ganapathi, P.; Ganesan, K. Anti-bacterial, catalytic and docking behaviours of novel di/trimeric imidazolium salts. J. Mol. Liq. 2017, 233, 452-464. [CrossRef]

140. Forman, M.E.; Jennings, M.C.; Wuest, W.M.; Minbiole, K.P.C. Building a Better Quaternary Ammonium Compound (QAC): Branched Tetracationic Antiseptic Amphiphiles. Chem. Med. Chem. 2016, 11, 1401-1405. [CrossRef]

141. Marafino, J.N.; Gallagher, T.M.; Barragan, J.; Volkers, B.L.; LaDow, J.E.; Bonifer, K.; Fitzgerald, G.; Floyd, J.L.; McKenna, K.; Minahan, N.T.; et al. Colloidal and antibacterial properties of novel triple-headed, double-tailed amphiphiles: Exploring structure-activity relationships and synergistic mixtures. Bioorg. Med. Chem. 2015, 23, 3566-3573. [CrossRef]

142. Gallagher, T.M.; Marafino, J.N.; Wimbish, B.K.; Volkers, B.; Fitzgerald, G.; McKenna, K.; Floyd, J.; Minahan, N.T.; Walsh, B.; Thompson, K.; et al. Hydra amphiphiles: Using three heads and one tail to influence aggregate formation and to kill pathogenic bacteria. Colloids Surf. B 2017, 157, 440-448. [CrossRef]

143. Al-Khalifa, S.E.; Jennings, M.C.; Wuest, W.M.; Minbiole, K.P.C. The Development of Next-Generation Pyridinium-Based multiQAC Antiseptics. Chem. Med. Chem. 2017, 12, 280-283. [CrossRef]

144. Vereshchagin, A.N.; Minaeva, A.P.; Egorov, M.P. Synthesis and antibacterial activity of new tetrameric quaternary ammonium compounds based on pentaerythritol and 3-hydroxypyridine. Russ. Chem. Bull. 2021, 70, 545-548. [CrossRef]

145. Kamber, N.E.; Jeong, W.; Waymouth, R.M.; Pratt, R.C.; Lohmeijer, B.G.G.; Hedrick, J.L. Organocatalytic Ring-Opening Polymerization. Chem. Rev. 2007, 107, 5813-5840. [CrossRef]

146. Matyjaszewski, K.; Spanswick, J. Controlled/living radical polymerization. Mater. Today 2005, 8, 26-33. [CrossRef]

147. Huang, D.; Qin, A.; Tang, B.Z. CHAPTER 1 Overview of Click Polymerization. In Click Polymerization; The Royal Society of Chemistry: Croydon, UK, 2018; pp. 1-35.

148. Lu, G.; Wu, D.; Fu, R. Studies on the synthesis and antibacterial activities of polymeric quaternary ammonium salts from dimethylaminoethyl methacrylate. React. Funct. Polym. 2007, 67, 355-366. [CrossRef]

149. Guo, J.; Qin, J.; Ren, Y.; Wang, B.; Cui, H.; Ding, Y.; Mao, H.; Yan, F. Antibacterial activity of cationic polymers: Side-chain or main-chain type? Polym. Chem. 2018, 9, 4611-4616. [CrossRef]

150. Badawy, M.E.I. Structure and antimicrobial activity relationship of quaternary N-alkyl chitosan derivatives against some plant pathogens. J. Appl. Polym. Sci. 2010, 117, 960-969. [CrossRef]

151. Shaban, S.M.; Aiad, I.; Moustafa, A.H.; Aljoboury, O.H. Some alginates polymeric cationic surfactants; surface study and their evaluation as biocide and corrosion inhibitors. J. Mol. Liq. 2019, 273, 164-176. [CrossRef]

152. Dizman, B.; Elasri, M.O.; Mathias, L.J. Synthesis and antimicrobial activities of new water-soluble bis-quaternary ammonium methacrylate polymers. J. Appl. Polym. Sci. 2004, 94, 635-642. [CrossRef]

153. Timofeeva, L.M.; Kleshcheva, N.A.; Moroz, A.F.; Didenko, L.V. Secondary and Tertiary Polydiallylammonium Salts: Novel Polymers with High Antimicrobial Activity. Biomacromolecules 2009, 10, 2976-2986. [CrossRef]

154. Kougia, E.; Tselepi, M.; Vasilopoulos, G.; Lainioti, G.C.; Koromilas, N.D.; Druvari, D.; Bokias, G.; Vantarakis, A.; Kallitsis, J.K. Evaluation of Antimicrobial Efficiency of New Polymers Comprised by Covalently Attached and/or Electrostatically Bound Bacteriostatic Species, Based on Quaternary Ammonium Compounds. Molecules 2015, 20, 21313-21327. [CrossRef]

155. Druvari, D.; Koromilas, N.D.; Lainioti, G.C.; Bokias, G.; Vasilopoulos, G.; Vantarakis, A.; Baras, I.; Dourala, N.; Kallitsis, J.K. Polymeric Quaternary Ammonium-Containing Coatings with Potential Dual Contact-Based and Release-Based Antimicrobial Activity. ACS Appl. Mater. Interface 2016, 8, 35593-35605. [CrossRef]

156. Bai, S.; Li, X.; Zhao, Y.; Ren, L.; Yuan, X. Antifogging/Antibacterial Coatings Constructed by N-Hydroxyethylacrylamide and Quaternary Ammonium-Containing Copolymers. ACS Appl. Mater. Interfaces 2020, 12, 12305-12316. [CrossRef]

157. Jaeger, W.; Bohrisch, J.; Laschewsky, A. Synthetic polymers with quaternary nitrogen atoms—Synthesis and structure of the most used type of cationic polyelectrolytes. Prog. Polym. Sci. 2010, 35, 511-577. [CrossRef]

158. Carmona-Ribeiro, A.M.; De Melo Carrasco, L.D. Cationic Antimicrobial Polymers and Their Assemblies. Int. J. Mol. Sci. 2013, 14, 9906-9946. [CrossRef]

159. Chen, A.; Peng, H.; Blakey, I.; Whittaker, A.K. Biocidal Polymers: A Mechanistic Overview. Polym. Rev. 2017, 57, 276-310. [CrossRef]

160. Jie, Z.; Yan, X.; Zhao, L.; Worley, S.D.; Liang, J. Eco-friendly synthesis of regenerable antimicrobial polymeric resin with $\mathrm{N}$-halamine and quaternary ammonium salt groups. RSC Adv. 2014, 4, 6048-6054. [CrossRef] 
161. Egorova, K.S.; Posvyatenko, A.V.; Larin, S.S.; Ananikov, V.P. Ionic liquids: Prospects for nucleic acid handling and delivery. Nucleic Acids Res. 2021, 49, 1201-1234. [CrossRef]

162. Ran, B.; Zhang, Z.; Yin, L.; Hu, T.; Jiang, Z.; Wang, Q.; Li, Y. A facile antibacterial coating based on UV-curable acrylated imidazoliums. J. Coat. Technol. Res. 2018, 15, 345-349. [CrossRef]

163. Torres, M.D.T.; Voskian, S.; Brown, P.; Liu, A.; Lu, T.K.; Hatton, T.A.; de la Fuente-Nunez, C. Coatable and resistance-proof ionic liquid for pathogen eradication. ACS Nano 2021, 15, 966-978. [CrossRef] [PubMed]

164. Zheng, Z.; Xu, Q.; Guo, J.; Qin, J.; Mao, H.; Wang, B.; Yan, F. Structure-antibacterial activity relationships of imidazolium-type ionic liquid monomers, poly(ionic liquids) and poly(ionic liquid) membranes: Effect of alkyl chain length and cations. ACS Appl. Mater. Interfaces 2016, 8, 12684-12692. [CrossRef] [PubMed]

165. Zhang, T.; Sun, B.; Guo, J.; Wang, M.; Cui, H.; Mao, H.; Wang, B.; Yan, F. Active pharmaceutical ingredient poly(ionic liquid)-based microneedles for the treatment of skin acne infection. Acta Biomater. 2020, 115, 136-147. [CrossRef]

166. Tejero, R.; Gutiérrez, B.; López, D.; López-Fabal, F.; Gómez-Garcés, J.; Muñoz-Bonilla, A.; Fernández-García, M. Tailoring macromolecular structure of cationic polymers towards efficient contact active antimicrobial surfaces. Polymers 2018, 10, 241. [CrossRef] [PubMed]

167. Sahiner, N.; Sagbas, S. Polymeric ionic liquid materials derived from natural source for adsorption purpose. Sep. Purif. Technol. 2018, 196, 208-216. [CrossRef]

168. Ethirajan, S.K.; Sengupta, A.; Jebur, M.; Kamaz, M.; Qian, X.; Wickramasinghe, R. Single-step synthesis of novel polyionic liquids having antibacterial activity and showing $\pi$-electron mediated selectivity in separation of aromatics. ChemistrySelect 2018, 3, 4959-4968. [CrossRef]

169. Claus, J.; Jastram, A.; Piktel, E.; Bucki, R.; Janmey, P.A.; Kragl, U. Polymerized ionic liquids-based hydrogels with intrinsic antibacterial activity: Modern weapons against a ntibiotic-resistant infections. J. Appl. Polym. Sci. 2020, 138, 50222. [CrossRef]

170. Fang, C.; Kong, L.; Ge, Q.; Zhang, W.; Zhou, X.; Zhang, L.; Wang, X. Antibacterial activities of N-alkyl imidazolium-based poly(ionic liquid) nanoparticles. Polym. Chem. 2019, 10, 209-218. [CrossRef]

171. Niesyto, K.; Neugebauer, D. Synthesis and characterization of ionic graft copolymers: Introduction and in vitro release of antibacterial drug by anion exchange. Polymers 2020, 12, 2159. [CrossRef] [PubMed]

172. Fang, H.; Wang, J.; Li, L.; Xu, L.; Wu, Y.; Wang, Y.; Fei, X.; Tian, J.; Li, Y. A novel high-strength poly(ionic liquid)/PVA hydrogel dressing for antibacterial applications. Chem. Eng. J. 2019, 365, 153-164. [CrossRef]

173. Fang, H.; Li, D.; Xu, L.; Wang, Y.; Fei, X.; Tian, J.; Li, Y. A reusable ionic liquid-grafted antibacterial cotton gauze wound dressing. J. Mater. Sci. 2021, 56, 7598-7612. [CrossRef]

174. Andresen, M.; Stenstad, P.; Møretrø, T.; Langsrud, S.; Syverud, K.; Johansson, L.-S.; Stenius, P. Nonleaching Antimicrobial Films Prepared from Surface-Modified Microfibrillated Cellulose. Biomacromolecules 2007, 8, 2149-2155. [CrossRef]

175. Song, J.; Kong, H.; Jang, J. Bacterial adhesion inhibition of the quaternary ammonium functionalized silica nanoparticles. Colloids Surf. B 2011, 82, 651-656. [CrossRef]

176. Liu, Y.; Ma, K.; Li, R.; Ren, X.; Huang, T.S. Antibacterial cotton treated with N-halamine and quaternary ammonium salt. Cellulose 2013, 20, 3123-3130. [CrossRef]

177. Liu, Y.; Li, J.; Cheng, X.; Ren, X.; Huang, T.S. Self-assembled antibacterial coating by N-halamine polyelectrolytes on a cellulose substrate. J. Mater. Chem. B 2015, 3, 1446-1454. [CrossRef]

178. Asri, L.A.T.W.; Crismaru, M.; Roest, S.; Chen, Y.; Ivashenko, O.; Rudolf, P.; Tiller, J.C.; van der Mei, H.C.; Loontjens, T.J.A.; Busscher, H.J. A Shape-Adaptive, Antibacterial-Coating of Immobilized Quaternary-Ammonium Compounds Tethered on Hyperbranched Polyurea and its Mechanism of Action. Adv. Func. Mater. 2014, 24, 346-355. [CrossRef]

179. Zhao, J.; Millians, W.; Tang, S.; Wu, T.; Zhu, L.; Ming, W. Self-Stratified Antimicrobial Acrylic Coatings via One-Step UV Curing. ACS Appl. Mater. Interface 2015, 7, 18467-18472. [CrossRef] [PubMed]

180. Zhang, X.; Ma, J.; Tang, C.Y.; Wang, Z.; Ng, H.Y.; Wu, Z. Antibiofouling Polyvinylidene Fluoride Membrane Modified by Quaternary Ammonium Compound: Direct Contact-Killing versus Induced Indirect Contact-Killing. Environ. Sci. Technol. 2016, 50, 5086-5093. [CrossRef] [PubMed]

181. Żywicka, A.; Fijałkowski, K.; Junka, A.F.; Grzesiak, J.; El Fray, M. Modification of Bacterial Cellulose with Quaternary Ammonium Compounds Based on Fatty Acids and Amino Acids and the Effect on Antimicrobial Activity. Biomacromolecules 2018, 19, 1528-1538. [CrossRef]

182. He, D.; Yu, Y.; Liu, F.; Yao, Y.; Li, P.; Chen, J.; Ning, N.; Zhang, S. Quaternary ammonium salt-based cross-linked micelle templated synthesis of highly active silver nanocomposite for synergistic anti-biofilm application. Chem. Eng. J. 2020, 382, 122976. [CrossRef]

183. Alkabli, J.; El-Sayed, W.N.; Elshaarawy, R.F.M.; Khedr, A.I.M. Upgrading Oryza sativa wastes into multifunctional antimicrobial and antibiofilm nominees; Ionic Metallo-Schiff base-supported cellulosic nanofibers. Eur. Polym. J 2020, 138, 109960. [CrossRef]

184. Xu, Q.; Zheng, Z.; Wang, B.; Mao, H.; Yan, F. Zinc ion coordinated poly(ionic liquid) antimicrobial membranes for wound healing. ACS Appl. Mater. Interfaces 2017, 9, 14656-14664. [CrossRef] [PubMed]

185. Liu, P.; Jin, K.; Wong, W.; Wang, Y.; Liang, T.; He, M.; Li, H.; Lu, C.; Tang, X.; Zong, Y.; et al. Ionic liquid functionalized non-releasing antibacterial hydrogel dressing coupled with electrical stimulation for the promotion of diabetic wound healing. Chem. Eng. J. 2021, 415, 129025. [CrossRef]

186. Jin, L.; Shi, Z.; Zhang, X.; Liu, X.; Li, H.; Wang, J.; Liang, F.; Zhao, W.; Zhao, C. Intelligent antibacterial surface based on ionic liquid molecular brushes for bacterial killing and release. J. Mater. Chem. B 2019, 7, 5520-5527. [CrossRef] 
187. He, X.; Yang, Y.; Song, H.; Wang, S.; Zhao, H.; Wei, D. Polyanionic composite membranes based on bacterial cellulose and amino acid for antimicrobial application. ACS Appl. Mater. Interfaces 2020, 12, 14784-14796. [CrossRef] [PubMed]

188. Guan, J.; Wang, Y.; Wu, S.; Li, Y.; Li, J. Durable anti-superbug polymers: Covalent bonding of ionic liquid onto the polymer chains. Biomacromolecules 2017, 18, 4364-4372. [CrossRef] [PubMed]

189. Raucci, M.G.; Fasolino, I.; Pastore, S.G.; Soriente, A.; Capeletti, L.B.; Dessuy, M.B.; Giannini, C.; Schrekker, H.S.; Ambrosio, L. Antimicrobial imidazolium ionic liquids for the development of minimal invasive calcium phosphate-based bionanocomposites. ACS Appl. Mater. Interfaces 2018, 10, 42766-42776. [CrossRef] [PubMed]

190. Suner, S.S.; Sahiner, M.; Akcali, A.; Sahiner, N. Functionalization of halloysite nanotubes with polyethyleneimine and various ionic liquid forms with antimicrobial activity. J. Appl. Polym. Sci. 2019, 137, 48352. [CrossRef]

191. Gindri, I.M.; Palmer, K.L.; Siddiqui, D.A.; Aghyarian, S.; Frizzo, C.P.; Martins, M.A.P.; Rodrigues, D.C. Evaluation of mammalian and bacterial cell activity on titanium surface coated with dicationic imidazolium-based ionic liquids. Rsc Adv. 2016, 6, 36475-36483. [CrossRef]

192. Ye, Q.; Gao, T.; Wan, F.; Yu, B.; Pei, X.; Zhou, F.; Xue, Q. Grafting poly(ionic liquid) brushes for anti-bacterial and anti-biofouling applications. J. Mater. Chem. 2012, 22, 13123-13131. [CrossRef]

193. Mehta, M.J.; Kumar, A. Ionic liquid assisted gelatin films: Green, UV shielding, antioxidant, and antibacterial food packaging materials. ACS Sustain. Chem. Eng. 2019, 7, 8631-8636. [CrossRef]

194. Martini Garcia, I.; Jung Ferreira, C.; de Souza, V.S.; Castelo Branco Leitune, V.; Samuel, S.M.W.; de Souza Balbinot, G.; de Souza da Motta, A.; Visioli, F.; Damiani Scholten, J.; Mezzomo Collares, F. Ionic liquid as antibacterial agent for an experimental orthodontic adhesive. Dent. Mater. 2019, 35, 1155-1165. [CrossRef] [PubMed]

195. O'Neill, J. Tackling Drug-Resistant Infections Globally: Final Report And Recommendations; Welcome Trust: London, UK, 2016 ; p. 84. 